\title{
Preliminary Prediction of Inflow into the D-Holes at the Stripa Mine
}

\author{
Jane C. S. Long, Kenzi Karasaki, Amy Davey, John Peterson, \\ Martin Landsfeld, John Kemeny, Stephen Martel
}

\author{
Earth Sciences Division \\ Lawrence Berkeley Laboratory \\ University of California \\ Berkeley, California 94720
}

February 1990

This work was supported by the Manager, Chicago Operations, Repository Tcchnology Program, Repository Technology and Transportation Division, of the U.S. Department of Energy under Contract No. DEACO3-76SF00098. 


\section{ABSTRACT}

Lawrence Berkeley Laboratory is contracted by the U.S. Department of Energy to provide an auxiliary modeling effort for the Stripa Project. Within this effor, we are making calculations of inflow to the Simulated Drift Experiment (SDE), i.e. inflow to six. parallel, closely spaced D-holes, using a preliminary set of data collected in five other holes, the N- and W-holes during Stages 1 and 2 of the Site Characterization and Validation (SCV) project. Our approach has been to focus on the fracture zones rather than the general set of ubiquitous fractures. Approximately $90 \%$ of all the water flowing in the rock is flowing in fracture zones (Ölsson et al 1989) which are neither uniformly conductive nor are they infinitely extensive. Our approach has been to adopt the fracture zone locations as they have been identified with geophysics. We use geologic sense and the original geophysical data to add one zone where significant water inflow has been observed that can not be explained with the other geophysical zones.

We superimpose a regular grid of conductors on the fracture zones. These could be considered "channels", but mathematically, the grid is simply a discretization of the plane. The grid elements are each assigned an equal conductance. Then we use crosshole hydrologic tests to condition the model with a technique called "simulated annealing". In simulated annealing, we simulate well tests using the model and compare the calculated results to the measured well test behavior. We then adjust the model by removing or replacing grid elements until the predicted heads are as close as possible to the observed ones. From annealing we get a series of models which all fit the hydrologic data to approximately the same degree of agreement. Annealing theory allows us to rank these according to their relative likelihood.

At the time this work was done, there were no systematic cross-hole well test data available for the SCV site. In order to test our approach, we have synthesized data for a cross-hole test from some informal cross-hole tests performed by the British Geologic Survey (BGS). In these tests, W2 was opened and responses were observed in the other holes. From this data, as well as the head and flow records in the holes, we have made a synthetic steady state well test record due to the opening of W2. We have annealed to this data to develop a prelininary estimate of a hydrologic model of the SCV site.

We then scale the conductance of the elements such that the model makes the best possible prediction of inflow rates which were observed in the $\mathrm{W}$ - and $\mathrm{N}$-holes. Finally, we close off the wells used to calibrate the model, open the D-holes and calculate inflow to the D-holes. Using this technique we predict a mean total flow of approximately 3.1 $(l / m i n)$ into the six D-holes with a coefficient of variation nearly unity. We estimated the flow to the D-holes five times, sequentially leaving one inflow measurement out of each calculation. The remaining tests would then be used to predict the flow into the hole left out. By then comparing the prediction to the measured result, a prediction error of about $4.6 \mathrm{l} / \mathrm{min}$ was calculated. This is an estimate of the error to be expected in the prediction of inflow. Based on preliminary analysis of the SDE experiment, the actual inflow is close to $2 \mathrm{l} / \mathrm{min}$.

In our calculation of flow into the D-holes, we have not differentiated flow between the D-holes. This is because the diameter of the ring of D-holes is about $3 \mathrm{~m}$ whereas the grid elements are about $10 \mathrm{~m}$ apart. By using the distribution of flows into the $\mathrm{N}$ - and W- holes, we followed a bootstrapping technique to estimate that the coefficient of variation for flows among the D-holes would be almost unity. This implies that one of the six holes could carry more than half the total flow. 
ABSTRACT iii

LIST OF FIGURES vii

LIST OF TABLES Xiij

ACKNOWLEDGMENTS $\quad \mathbf{x V}$

$\begin{array}{lll}\text { 1.0. INTRODUCTION } & 1\end{array}$

1.1. Background I

1.2. Goals 1

1.3. Validation 7

1.4. Approach to Fracture Flow Modeling 8

2.0. THE FRACTURE ZONE MODEL 11

2.1. Identification of Fracture Zones with Geophysics 11

2.2. The Hydrologic Conceptual Model 12

2.3. Geomechanical Interpretation of the Shear Zones 22

2.3.1. Fractures in the SCV Block 24

2.3.2. Geomechanical Modeling of the Shear Zones 26

$\begin{array}{lll}\text { 2.4. Conclusions } & 27\end{array}$

3.0. THE NUMERICAL MODEL 31

3.1. The CHANGE Model 31

3.2. TRINET 31

3.3. Scale of Analysis ("Resolution") 32

3.4. Treatment of the D-Holes as One Big Hole 32

3.5. Connecting the Zones to the Boreholes 32

4.0. SIMULATED ANNEALING 35

4.1. Analogy with Metal Annealing 36

4.2. Bayesian Analysis $\quad 38$

4.3. The Annealing Computer Algorithm 39

4.4. A Synthetic Example Case 40 
5.0. MEASURES OF UNCERTAINTY 43

5.1. Prediction Enor 43

5.2. Sensitivity and Relative Likelihood 44

6.0. PREDICTION OF INFLOW TO THE D-HOLES 47

6.1. Development of a Synthetic Cross-hole Test 47

6.2. Boundary Conditions and the Starting Configuration 52

6.3. The Energy Versus Iteration Curve 62

6.4. Calibration to W2 Flow (Shifting) 64

6.5. Modification of the Prediction Using Other Inflows 73

6.6. Estimation of Prediction Error 74

6.7. Estimate of Distribution of Flow Between D-Holes 74

7.0. SUMMARY OF RESULTS AND CONCLUSIONS 79

8.0. EXPECTATIONS FOR FUTURE WORK 81

8.1. Excavation Inflow 81

8.2. Stress Effects $\quad 83$

8.3. Water Pressure Effects 83

8.4. Blasting Effects 85

8.5. Ventilation Effects 85

8.6. Conclusion 85

$\begin{array}{lll}\text { 9.0. REFERENCES } & 87\end{array}$

APPENDIX A: FRACTAL ANALYSIS 91

APPENDIX B: THE GRID MODEL 97 


\section{LIST OF FIGURES}

\section{Page}

Figure 1.1. Plan view of the SCV site. 2

Figure 1.2. Outline of the investigation programme known as the "Simu- 3 lated Drifi Experiment" including eventual drifi excavation.

Figure 1.3. Plan view of the SCV site showing the location of the C-holes and the Validation Drift.

Figure 1.4. Cross-section of the SCV site looking North showing the Choles and the Validation Drift. Gha, Ghb, and GB are fracture zones identified with geophysics.

Figure 1.5. Perspective view of the SCV block. Dotted area in the upper left is the mined out stopes (after J. Gale).

Figure 2.1a. Summary data sheet for W1. Hydraulic conductivities greater than $10^{-8} \mathrm{~m} / \mathrm{s}$ have been blackened in. Geophysical features are shown as shaded horizontal bands. Hydrologic zones are marked with " $I$ " bars in the right hand column.

Figure 2.1b. Summary data sheet for W2. Hydraulic conductivities greater than $10^{-8} \mathrm{~m} / \mathrm{s}$ have been blackened in. Geophysical features are shown as shaded hurizontal bands. Hydrologic zones are marked with " $I$ " bars in the right hand column.

Figure 2.1c. Summary data sheet for N2. Hydraulic conductivities greater than $10^{-8} \mathrm{~m} / \mathrm{s}$ have been blackened in. Geophysical features are shown as shaded horizontal bands. Hydrologic zones are marked with " $I$ " bars in the right hand column.

Figure 2.1d. Summary data sheet for N3. Hydraulic conductivities greater than $10^{-8} \mathrm{~m} / \mathrm{s}$ have been blackened in. Geophysical features are shown as shaded horizontal bands. Hydrologic zones are marked with " $I$ " bars in the right hand column. 
Figure 2.1e. Summary data sheet for N4. Hydraulic conductivities greater than $10^{-8} \mathrm{~m} / \mathrm{s}$ have been blackened in. Geophysical features are shown as shaded horizontal bands. Hydrologic zones are marked vith "I" bars in the right hand column.

Figure 2.2. A perspective view of the SCV block looking up to the NorthEast showing zones $B$ and $C$ and that two hydrologic anomalies lie between these zones in a plane parallel to them.

Figure 2.3. The hydrologic zone model shown in perspective from the North-West looking down. Zones A, B, B', C, Ha, $\mathrm{Hb}$ and I are shown. Gridding on the planes represents the hydraulic conductors of the template used for annealing.

Figure 2.4. Residual radar slowness tomogram for the borehole section N3N4 made with a center frequency of $22 \mathrm{MHz}$.

Figure 2.5. Hypothetical testing zones in a fractured rock showing that the transmissivity measured in the three zones will overiap such that the transmissivity measured separately will add up to more transmissivity than the true total.

Figure 2.6. Poles to the fractures in zone GB' (upper hemisphere) where it intersects borehole $\mathrm{N} 4$ (depths $80-95 \mathrm{~m}$ ).

Figure 2.7. Poles to the fractures in zone GH (upper hemi-phere where it intersects borehole W1 (depths $48-63 \mathrm{~m}$ ).

Figure 2.8. (a) Nine randomly distributed fractures subjected to the maximum horizontal and vertical stresses on the $360 \mathrm{~m}$ level (b) Two long fractures representing the slickensided fractures in the NE-striking zones are added. Table lists average Modes I and II stress intensity factors for each crack for the two cases.

Figure 3.1. The hydrologic zone model with the fins that connect the zones to the boreholes.

Figure 4.1. (a) A "real" fracture network where we have generated "real" data on a well test in hole $A$ and monitoring in holes B through G; (b) Template model; (c) The pattem of conductors resulting from annealing.

Figure 6.1. Responses in the SCV block caused by opening W2. Three different tests were performed, sequentially monitoring the responses in N4, N3 and W1. In the case of N4 and N3, packers were placed in the holes so that different responses could be seen in different parts of the well. In the case of Wl, the hole was undivided (after Holmes, in Olsson, 1988). 
Figure 6.2a. (a) Response to N3 to opening W2. (b) Response in N4 to opening W2. (c) Response in W1 to opening W2.

Figure 6.3. Flowrale in W2 recorded during two periods: when N3 was monitored and when W1 was monitored.

Figure 6.4. Responses in N3, N4 and W1 (entire hole) to opening W2 (from the head records, 1987).

Figure 6.5. Summary of the synthetic annealing case.

Figure 6.6. Heads in the SCV block (after Olsson, 1988).

Figure 6.7. Heads that result in the $\mathbf{N}$ - and $\mathbf{W}$-holes when $\mathbf{W} 2$ is opened and head $=200 \mathrm{~m}$ boundary conditions are applied directly to the SCV block boundaries.

Figure 6.8a. Perspective plot showing how the zone $A$ is connected to the boundaries of the model which are held at $200 \mathrm{~m}$ head.

Figure 6.8b. Perspective plot showing how the zone $B$ is connected to the boundaries of the model which are held at $200 \mathrm{~m}$ head.

Figure 6.8c. Perspective plot showing how the zone $\mathrm{B}^{\prime}$ is connected to the boundaries of the model which are held at $200 \mathrm{~m}$ head.

Figure 6.8d. Perspective plot showing how the zone $\mathrm{C}$ is connected to the boundaries of the model which are held at $200 \mathrm{~m}$ head.

Figure 6.8e. Perspective plot showing how the zone $\mathrm{Ha}$ is connected to the boundaries of the model which are held at $200 \mathrm{~m}$ head.

Figure 6.8f. Perspective plot showing how the zone $\mathrm{Hb}$ is connected to the boundaries of the model which are held at $200 \mathrm{~m}$ head.

Figure 6.8g. Perspective plot showing how the zone I is connected to the boundaries of the model which are held at $200 \mathrm{~m}$ head.

Figure 6.9. Record of energy versus iteration for the SCV synthetic well test case.

Figure 6.10a. Grid elements in zone $A$. The top is the full template, the middle is the initial configuration, and the bottom is the final configuration after annealing. 
Figure 6.10b. Grid elements in zone B. The top is the full template, the middle

is the initial configuration, and the bottom is the final configuration after annealing.

Figure 6.10c. Grid elements in zone $\mathbf{B}^{\prime}$. The top is the full template, the middle is the initial configuration, and the bottom is the final configuration after annealing.

Figure 6.10d. Grid elements in zone $C$. The top is the full template, the middle is the initial configuration, and the bottom is the final configuration after annealing.

Figure 6.10e. Grid elements in zone Ha. The top is the full template, the middle is the initial configuration, and the bottom is the final configuration after annealing.

Figure 6.10f. Grid elements in zone $\mathrm{Hb}$. The top is the full template, the middle is the initial configuration, and the bottom is the final configuration after annealing.

Figure 6.10g. Grid elements in zone $I$. The top is the full template, the middile is the initial configuration, and the bottom is the final configuration after annealing.

Figure 6.11. The distributions used to make an estimate of the variation in flow between the D-holes. (a) Empirical density of hydraulic conductivity from the 10 most permeable zones in the $\mathrm{N}$ and $\mathrm{W}$ boreholes. (b) Estimated density of flow variance between the individual D-holes based on bootstrapped sampling of the empiical distribution of conductivities and the estimate of total $D$ hole flow.

Figure 8.1. Distance-drawdown plot of the $20^{\circ}$ test results of the macropermeability experiment.

Figure 8.2. Flow diversion due to de-pressurization.

Figure A.1. A partially filled rectangular three-dimensional grid which could be used for co-annealing with geophysics.

Figure A.2. A cartoon showing a hydrologic zone intersecting the plane of a tomogram. Fow is not confined to the plane of the tomogram, but the tomogram gives information about variability within the bydrologic feature. 
Figure A.3. Ray paths through a network showing how the number of conductors intersected by a ray can be counted.

Figure B.1. Drawdown curves for systems of partial dimension (after Barker, 98 1988).

Figure B.2. Physical systems where flow between wells A and B would be one-dimensional, two-dimensional, and of partial dimension respectively.

Figure B.3. A random fractal mesh and the response curves for a well test on 99 this mesh. 


\section{LIST OF TABLES}

Page

$\begin{array}{ll}\text { Table 2.1. Geophysical Fracture Zones } & 12\end{array}$

$\begin{array}{lll}\text { Table 2.2. Hydraulic Transmissivity Distribution } & 18\end{array}$

Table 6.1. Annealed Borehole Sections and Head Values 52

Table 6.2. Annealing results at the final iteration $=3749 \quad 72$

Table 6.3. Inflow to the D-Holes Calculated for Ten Different 72

Configurations of the Model

Table 6.4. Open Hole Inflows to $\mathrm{N}$ - and W-Holes 73

Table 6.5. Predictions of D-Hole Inflow Based on Annealing and Measured 74 $\mathrm{N}$ - and $\mathrm{W}$-Hole Inflows

$\begin{array}{lll}\text { Table 6.6. } & \text { Prediction Error } & 75\end{array}$

Table 6.7. Partition of the Expected Flow in the D-Holes by Zones 75

Table B.1. Slope of Drawdown Curves for Networks of Different Fractal $\quad$ i00 Dimension 


\section{ACKNOWLEDGMENTS}

This work was supported by the Repository Technology and Transporation Division of the Office of Civilian Nuclear Waste Management of the U.S. Department of Energy under contract DEACO3-76SF00098. We are grateful for their support. We also wish to thank the Stripa Project for accommodating and supporting our efforts. In particular, we would like to thank Ned Patera, Mike Ferrigan, Olle Olsson, John Black, Bengt Stillborg, Paul Gnirk, David Holmes, Calin Cosma, Jurgin Pihl, John Gale, Bill Dershowitz, Alari rierben, Gunnar Gustafson, David Hodg. kinson, and Stratis Vomvoris for their help. discussions and encouragement. We would like to thank Paul Witherspoon, Joe Wang, David Hodgkinson and Veikko Taivassalo for reviewing this document. Last but not least, we thank our families for Lneir patience during our many, seemingly endless sojourns abroad. 


\subsection{INTRODUCTION}

\subsection{Background}

Investigations related to the geologic storage of nuclear waste have been ongoing at the Stripa Mine in Sweden for more than ten years. The latest of these investigations is called Phase II and is sponsored by OECD Nuclear Energy Association (NEA) as an intemational cooperative effort managed by the Swedish Nuclear Fuel and Waste Management Company (SKB).

The Stripa Phase III project includes the Site Characterization and Validation (SCV) experiment, which is designed to test current abilities to characterize fractured rock before it is used for nuclear waste storage. The effort is centered on a block of rock $150 \mathrm{~m}$ long $\times 100 \mathrm{~m}$ wide $\times 50 \mathrm{~m}$ deep in size at a depth of about $330 \mathrm{~m}$. The block lies beiween previous experimental sites, the Macropermeability/Bu ffer Mass Test site and the 3-D Migration site (Figure 1.1.). The SCV work is divided into two cycles of three stages each: data-gathering, prediction, and validation. The first stage of work has included drilling of 6 boreholes (N2,N3,N4, W1, W2, and V3) and measurements of geology, fracture characteristics, stress, single borehole geophysical logging, radar, seismics, and hydrogeology (Olsson et al., 1988a). Olsson et al. give background information describing the site and the results of the first cycle of data-gathering and prediction.

Lawrence Berkeley Laboratory (LBL) is contracted by the U.S. Department of Energy to paricipate in the hydrologic modeling of the SCV site. To that end, LBL provides three types of studies for the Stripa Project:

1. Hydrologic modeling support to other Stripa Project investigators. This may take the fom of parameter siudies used to help design the experimental program.

2. Calculation of flow and transport based on fracture network modeling.

3. Limited data collection and analysis when data which we find necessary to our modeling approach is not collected by the Stripa Project.

This is the first report of our efforts in fracture network modeling. The calculations presented here are based almost entirely on the information documented in Olsson et al. (1988a). Other information came from our efforts under 3. and through personal communication with the SCV principal investigators.

\subsection{Goals}

Hydrologic prediction of groundwater flow and transport is part of the work scope for the Stripa Phase III Project (SKB, 1987). Current plans call for two prediction cycles. The first cycle is focussed on the prediction of inflow into a clustered group of boreholes (the D-holes) which are drilled on the periphery of the future "Validation Driff". The D-holes are drilled such that five of them lie on the periphery of the Validation Drift and one lies in the center (Figures 1.2, 1.3, 1.4, and 1.5). Thus the measurement of flow into the D-holes is called the Simulated Drift Experiment. In the second cycle, the Validation Drift is excavated and inflow into the drift will be predicted. In both cases, actual measurements of inflow will be compared to the predictions. 


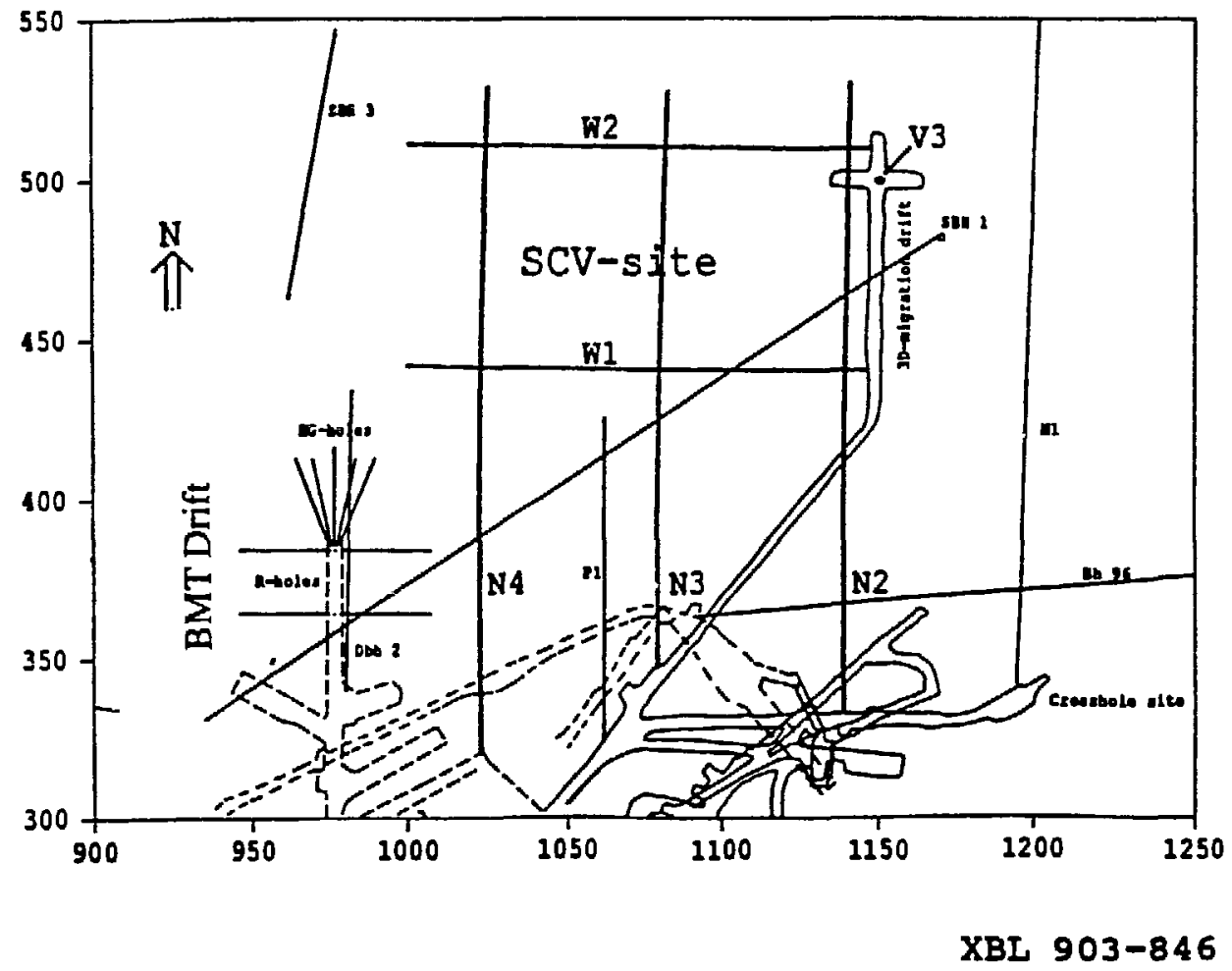

Figure 1.1. Plan view of the SCV site. 


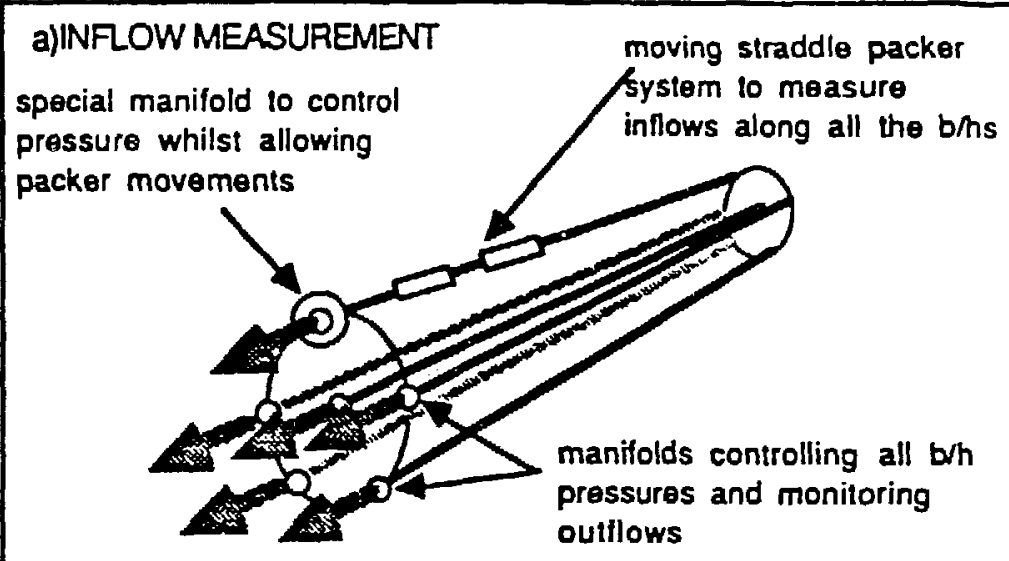

b) INFLOW DATA

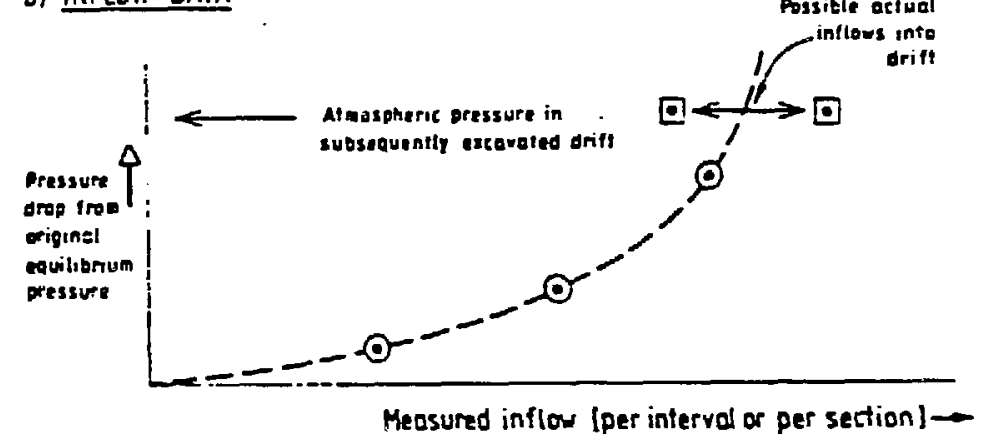

c) EXCAVATED DRIFT

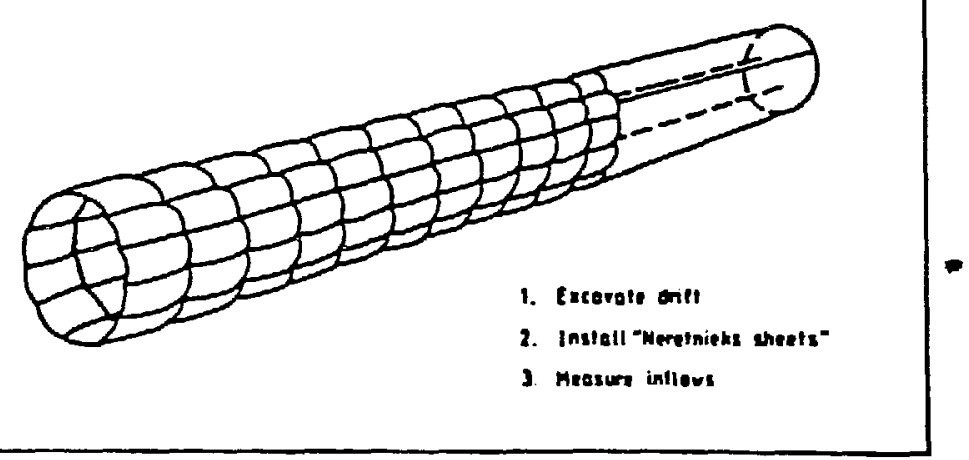

Figure 1.2. Outline of the investigation programme known as the "Simulated Drift Experiment" including eventual drift excavation. 


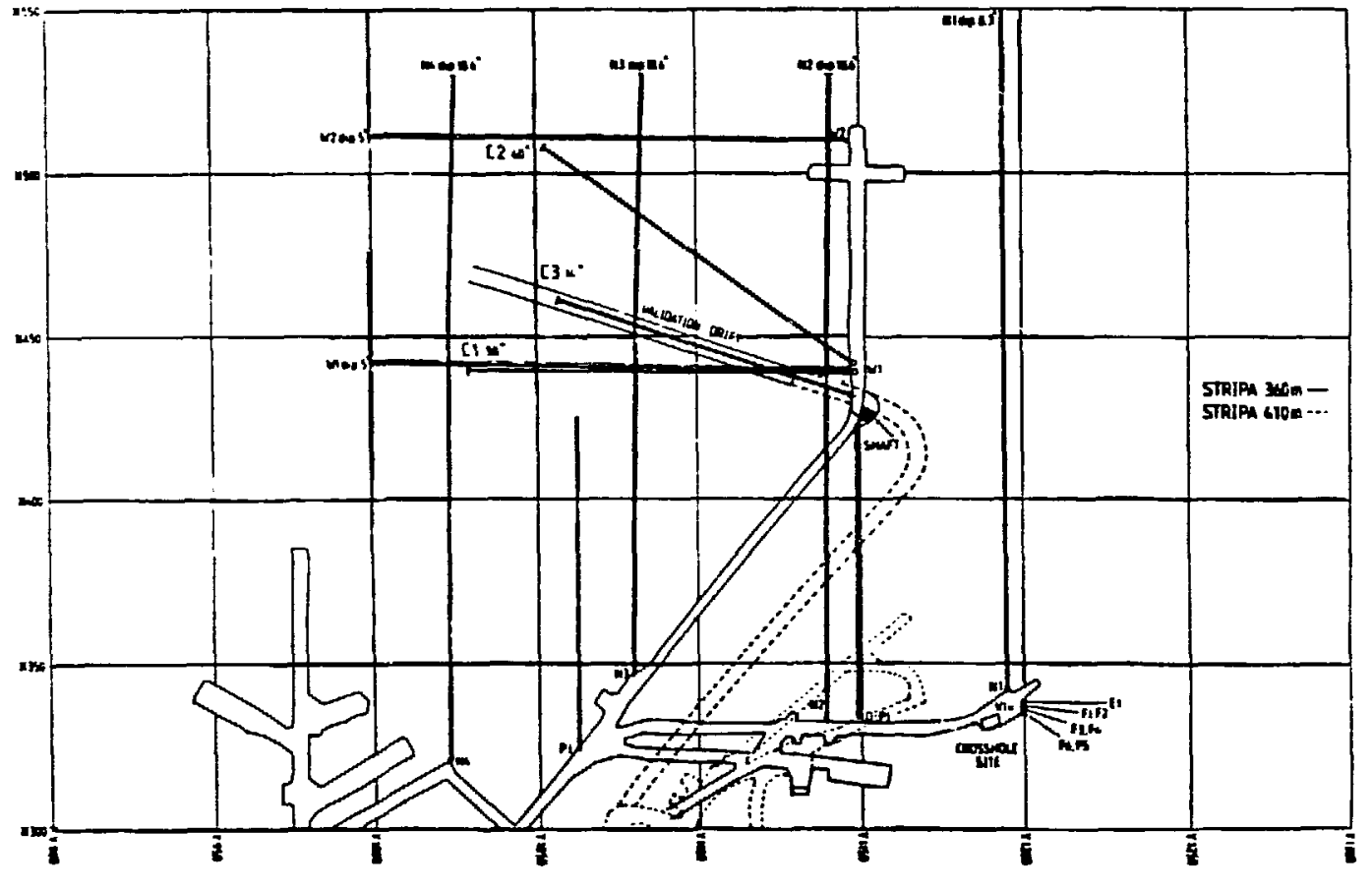

XBI $903-847$

Figure 1.3. Plan view of the SCV site showing the location of the C-holes and the Validation Drift. 


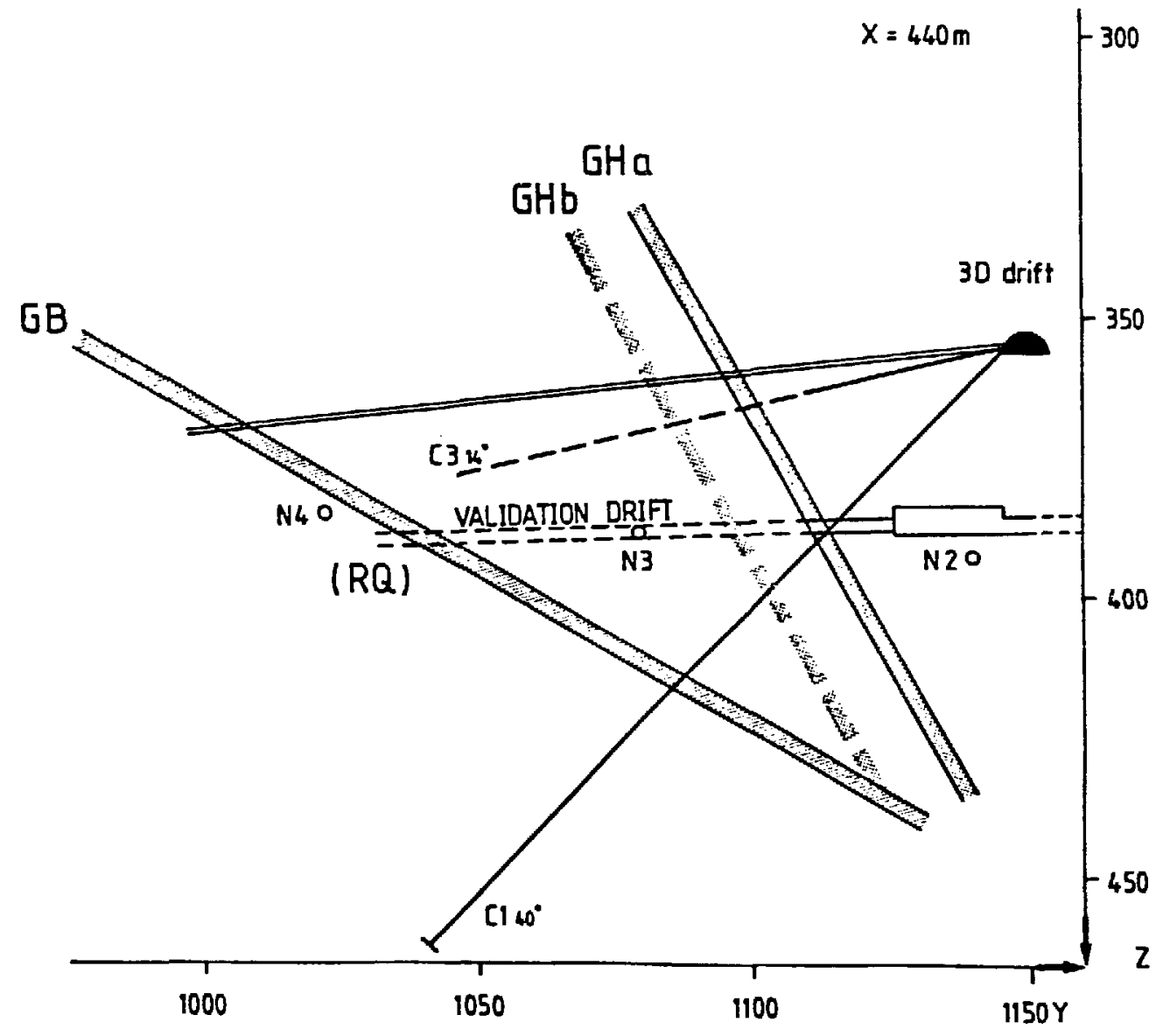

XBL 903-848

Figure 1.4. Cross-section of the SCV site looking North showing the C-holes and the Validation Drift. GHa. GHb, GB are fracture zones identified with geophysics. 


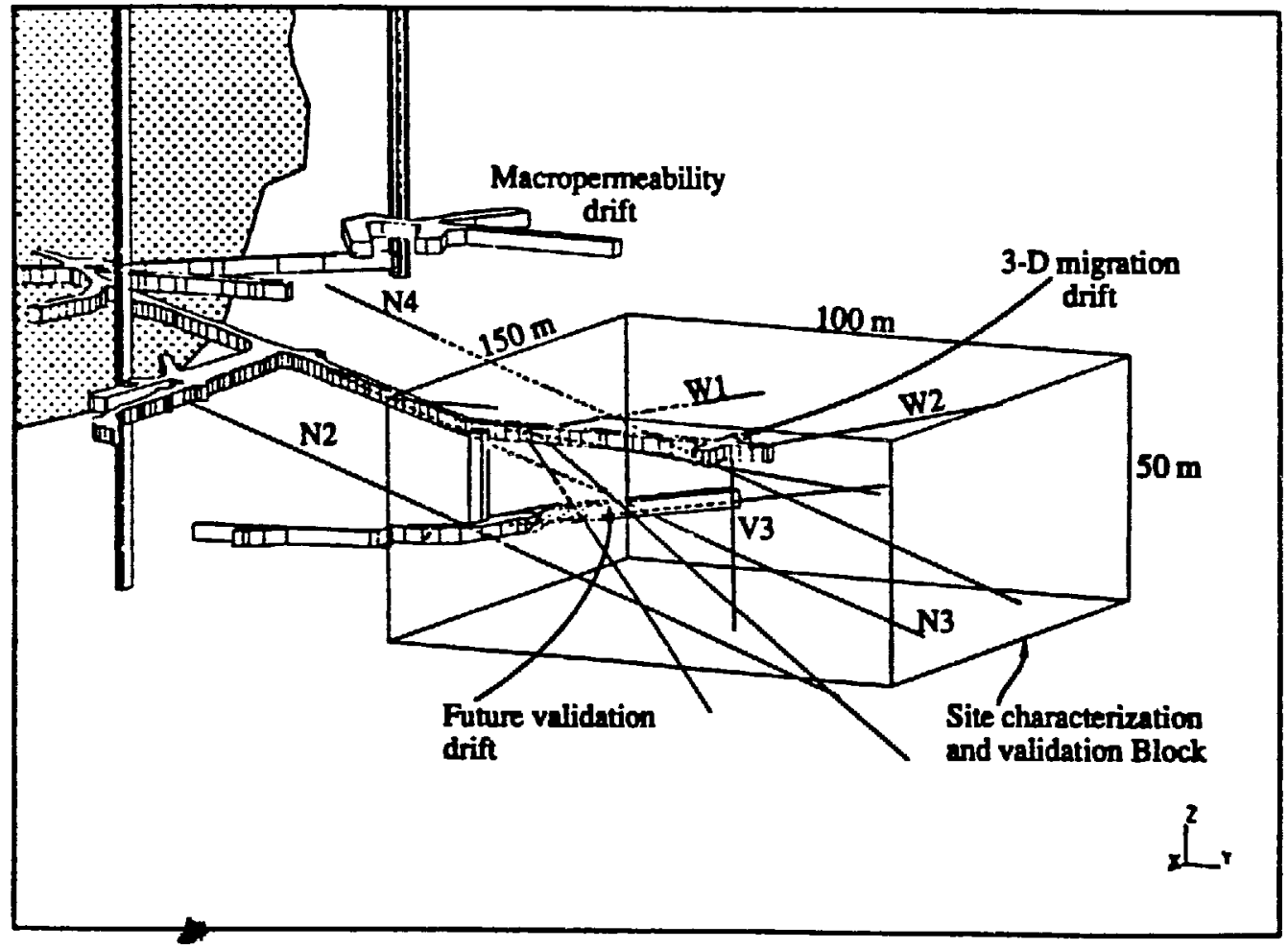

XBL $903-849$

Figure 1.5. Perspective view of the SCV block. Dotted area in the upper left is the mined out stopes (after J. Gale). 
This report covers LBL's preliminary prediction of flow into the D-holes. Care should be taken in interpreting the results given in this report As explained below, the approach that LBL has designed for developing a fracture hydrology model requires cross-hole hydrologic data. Cross-hole tests are planned for Stage 3 but were unavailable in Stage 1. As such, we have inferred from available data what a cross-hole test might show and used this synthetic data to make a preliminary calculation of the inflow into the D-holes. At a later date we will use the actual cross-hole tests to re-calculate inflow into the D-holes. Then using all the Stage 3 data we will calculate flow into the Validation Drift itself. The report mainly demonstrates the use of our methodology and the simulated results should be considered preliminary.

The following preliminary calculations are presented here:

1. Total flow into the six D-holes and measures of the uncertainty associated with this calculation

2. The distribution of this flow along the boreholes and measures of the uncertainty associated with this calculation

3. An estimate of the variability of flow between the six D-boreholes.

These quantities will be compared to the measured quantities as part of the "validation" of the flow model.

Chapters 1 through 5 of this report document background, approach, tools and the model construction. Chapter 6 gives the results, 7 the conclusions and 8 comments on future work. The reader wishing to go directly to results can skip to Chapter 6.

\subsection{Validation}

In the U.S. nuclear waste storage program, model validation has been taken to mean the establishment of the soundness of the models and the legitimacy of specific applications being made of those models. What actually constitutes validation is not a trivial matter. Our approach to validation has two components.

The first component is the calculation of prediction error. That is, we use the model to make a series of predictions. For each prediction we obtain a prediction error by comparing the calculated result to the measured result. The root mean square of these errors is called the prediction error (see Section 5.1 below). Thus, we validate the model by estimating how well the model makes predictions. For example, we could use the model to predict the inflow into ten different boreholes for which the inflow had been measured. By comparing the measurements of inflow to the predicted values, we can calculate a prediction error. Now, we can use the model to predict the flow into an eleventh hole for which the inflow is not known. The prediction error then provides an estimate of how good this prediction is, i.e. how valid the model is.

This approach is straight forward. The more numerous and diverse types of predictions that can be included in the estimate of prediction error, the more stringently "validated" 'the model can be. Although the model may work well to predict flow under one type of boundary condition, it may not predict well for a different type of boundary conditions. However, if it predicts well for two sets, then it is more likely to predict well under the third, even better for three, etc., etc.

A limitation of this approach to validation is that one is unlikely to have a sufficiently extensive set of insitu tests to compare models against. Thus, one rarely has a good 
statistical sample with which to estimate prediction error. Further, we often must extend the use of the models to classes of physical conditions, phenomena or time scales which we have not been able to test in the field. For example, one may wish to use a model which was "validated" "for drift inflow to predict large scale regional flow, or we may want to use the flow model as the basis for a transport prediction. Here the prediction error we can calculate does not apply to the problem at hand. Although, there is an expectation that validating a model for a given flow case is a first step in validating a model for other flow conditions or transport, a model validated for inflow is not necessarily a valid model for radically different boundary conditions or different processes.

This brings us to the second component of validation. This approach is commonly called "peer review" or "confidence building". In this type of validation, the modeling approach is scrutinized. What assumptions were made? Do they make sense? What is the evidence supporting these assumptions? What data were used? What is the sensitivity of the calculation to poor assumptions or inaccuracies in data? Which are the parameters that control the result?

This type of valioation is an iterative and a subjective process. Some of the assumptions cannot be checked easily for the simple reason that it would take infinite time to test all possible cases: i.e., one can prove a model is wrong, but you cannot prove it is right. We can only ask if the model is consistent with observations of all types that were made on the rock mass. Is the model consistent with the geologic observations, the geophysical measurements, the hydraulic measurements, etc.? For example, although the primary prediction is aimed at flux, we can also check to see if the calculated head distribution are consistent with the observed head distribution.

The approach to fracture flow modeling presented here is mindful of both kinds of validation. In Section 5 we explain in detail how prediction error is calculated and we use all the available appropriate data to do this. Below, we present a brief history of our approach to fracture flow modeling which tells how we try to incorporate as much of the physical observations as possible into the model so that we try to insure that the model is consistent with observations a priori. Finally, the physical assumptions made in the numerical code are spelled out in Section 3 and as actual calculations are presented below in Section 7 where we point out what parameters have the most control over the answer that we get.

\subsection{Approach to Fracture Flow Modeling}

The role of groundwater flow models is to make hydraulic predictions. Thus, in developing a model for fracture hydrology, we have concentrated on developing a model based on hydraulic behavior. This may sound trivial, but most fracture hydrology models are based on fracture geometry rather than hydraulic behavior. Going directly from the behavior to the model implies the use of inverse techniques and this is not a simple thing to do when dealing with fracture networks. First, we briefly review the types of accessible information for developing a fracture hydrology model. Then, we will briefly discuss our previous experience in modeling and the new approach that we are following with hydraulic, geophysical, and geologic data.

Data available for fracture hydrology models might include:

1. Fracture trace maps from drift walls or surface exposures giving fracture location, orientation, trace length, coatings, roughness etc. 
2. Fracture logs from boreholes giving fracture location, orientation, coatings, roughness etc.

3. Bore-hole geophysical logs

4. Single-hole packer tests

5. Cross-hole and reflection geophysics

6. Cross-hole hydraulic tests

The fundamental problem in modeling fracture hydrology is that the medium does not necessarily behave as a continuum. Parts of the rock have no hydraulic communication with other parts. In the case where the matrix rock can be considered impermeable, this fundamental behavior is govemed by the geometry of the fracture network. If a connected cluster of conductive fractures links point $A$ and point $B$, there can be flow between the two points. If not, then there is no flow. This heterogereous connectivity is very commonly observed in fractured rock including the Stripa rock. Our first attempts at building a fracture hydrology model were focussed on reproducing, a least in a statistical sense, the geometry of the fracture network. This involved determining a statistical rule for locating fractures, determining their orientation, size and conductivity. Then the interconnections between the fractures could be found and flow pattems calculated.

Such an interpretation was made of the data at the Fanay-Augères mine in France (Billaux et al. 1989). Data used included categories 1, 2 and 4 listed above. We assumed the fractures were disc-shaped and uniformly permeable in their plane. In order to represent the clustering of fractures, the arrangement of fractures in space was modeled using a determined by a complex Poisson process called the Parent-Daughter process. Data on trace lengths, orientation and fracture frequency were used to predict a pattem of fractures in a $100 \mathrm{~m}$ cube.

Conclusions from this effort were very striking. First, we found that the use of one- and two-dimensional data (i.e. borehole and trace-plane data) to infer unique threedimensional fracture geometry is an impossible task. Many different three-dimensional geometries can account for the same observations of one- and two-dimensional data. Second, no matter how the three-dimensional geometry was determined, if all the fractures present were hydrologically active, then the medium would have behaved like an equivalent continuum. However, cross-hole hydrologic and tracer test results showed that there were zones in the rock which simply did not respond to hydrologic stress from other zones. Therefore, the medium was definitely not behaving like an equivalent continuum and our analysis produced far too many fractures to account for the lack of connectivity known to exist.

In order to make this geometric approach successful, the three-dimensional geometry must be constrained. More importantly, a way must be found to account for the parts of the fracture system which do not conduct water. As to the three-dimensional geometry, we suspect that in many cases, one can show that fractures are relatively pervasive and the real problem is finding what parts of the system actually conduct.

Fanay-Augères offered one other key fact in this regard. Two drifts were mapped in this mine: one wet, one dry. For both drifts the fracture geometry seemed to indicate highly connected fracture networks. However, in the wet drift, a major fault ran through the block of rock surrounding this portion of the drift. It seems that the hydrology of the site is controlled by major features i.e., fracture zones. This observation is cerainly not confined to Fanay-Augères. As Olsson et al. (1988a) succinctly state, $94 \%$ of the hydraulic transmissivity is found in $4 \%$ of the tested rock in the SCV block. 
Similar evidence exits at the Underground Research Laboratory (URL) in Canada and other sites. This provides strong evidence for the role of fracture zones in controlling the hydrology. The hydrologic role of fracture zones also can explain the non-uniform cross-hole hydraulic responses such as observed in the SCV block. For example, the W2 hole evidenty intersects a large number of fracture zones. While the W2 hole at Stripa was being drilled, the observed heads in the entire SCV site dropped significantly and a similar response is observed each time W2 is opened or closed. However, the drilling or opening of other holes had little effect (Carlsten et al., 1988).

These facts imply that the nydrology of the SCV site is controlled by a finite number of major conductors that form a three-dimensional network of unknown configuration. In fact, it seems very likely that there are a few major features that dominate the entire hydrology of the site.

In conclusion, our experience so far has indicated that focussing on the details of fracture geometry statistics is equivalent to "not seeing the forest for the trees". If fracture zones control the hydrology, then efforts should first be aimed directly at locating and characterizing fracture zones. Further, one should concentrate on determining the hydrologic characteristics of the zones. We expect that zones are not continuous and that the permeability structure within the zones is complex. Therefore, we propose to build a model which concentrates on identifying the location of zones and the nature of permeability within the zones. In modeling the hydrology of the SCV site, therefore, we choose to model only the major hydrologic features. We assume that these features are associated with the fracture zones. A fracture zone is then assumed to consist of a two- dimensional network of hydrologic conductors.

To locate the zones, we rely heavily on the recent advances in geophysics that have allowed fractures zones to be "seen" inside the rock (Section 2.1). Then, based on the geophysical information we build a hydrologic conceptual model of the the rock which we call a template (2.2). The template should contain all the likely major conductors as is the basis for fluid flow calculation described in Section 3. We infer as much as possible about the qualitative hydrologic attributes of the zones through geology and geomechanics (2.3). Finally, we throw away conductors in a manner that conditions the model to observed well test behavior. In other words, within the template we identify pattems of conductance that can explain the observed hydraulic behavior. To do this we use simulated annealing to arrange the conductances such that they explain observed distributions of head, observed fluxes, or observed tracer test results (Section 4). This is a summary of the model building approach that was applied to the Stripa data. One other related approach, called the grid model, is under development and is briefly described in Appendix A. Section 6 then describes the synthetic data used in the annealing process; and gives the annealing results and the predictions of D-hole inflow. Another possible approach is described in Appendix B. Here, we could identify the fractal dimension of flow using Barker's (1988) analysis and relate this to the connectivity, and the connectivity to the percentage of conductances present in the template. This has not yet been done but is described in Appendix B. 


\subsection{THE FRACTURE ZONE MODEL}

\subsection{Identification of Fracture Zones with Geophysics}

Radar and seismic (acoustic) techniques were used at the SCV site to locate fracture zones. Hydrologic features such as fracture zones represent zones of increased porosity and water content and have different mechanical properties than the matrix rock and these property contrasts should be discemible with geophysics. Geophysical techniques may also find features which are not hydraulically important so it is important to use the geophysical information in conjunction with a geologic and hydrologic investigation.

Radar signals are sensitive to changes in dielectric constant. $E$ and electrical conductivity, $\sigma$ (Sen, 1981). Radar velocity is a function of $c / \sqrt{\mu / \varepsilon}$, where $c$ is the electromagnetic wave velocity. The amplitude is a function of $(\sigma / 2) \sqrt{\mu / \varepsilon}$, where $\mu$ is the electrical permeativity. The values of $c$ and $\mu$ are always constant, so a slowness tomogram maps contrasts in $\sqrt{\varepsilon}$ and an attenuation tomogram maps contrasts in $\sigma / \sqrt{\varepsilon}$.

Both the electrical conductivity, $\sigma$, and dielectric constant, $\varepsilon$, increase with the water content. So, the slowness tomogram should give the most direct correlation with fluid paths in the medium because it depends only on contrasts in $\varepsilon$. However, $\sigma$ varies over orders of magnitude whereas $\varepsilon$ only varies by a few percent. As a result, the attenuation tomograms are subject to fewer errors and the image is more clear.

The velocity of acoustic waves in rock are sensitive to changes in mock density, and mechanical properties such as bulk modulus. These are affected by porosity and water content as well as fracture stiffness. The attenuation of acoustic waves is a complex phenomena. Basically, there are two components to attenuation: intrinsic attenuation which is frictional energy loss, and scattering which is due to reflections and refractions at inhomogeneities. Fracture zones increase both types of attenuation of acoustic waves. So, a highly fractured region of the rock is expected to attenuate the seismic waves and also slow them dowr.

For both radar and seismics, both cross-hole tomographic and reflection techniques were used at Stripa. The tomographic technique uses the direct pulse, or first arrival information. The reflection analysis uses the later arrivals. Thus reflection data provide additional independent information about the fracture zones. Single-hole reflection data are used to locate reflectors in the same manner as common surface reflection surveys. It is possible to determine where the reflectors intersect the borehole and their orientation relative to the boreholes. This information provides a locus of possible reflectors which is a cone passing through this intersection between the boreholes and the reflectors. Use of a directional antenna will make it easier to determine the actual orientation of the feature. A cross-hole reflection technique which is similar to the single-hole method was also used.

An integrated analysis of the radar data was performed by Olsson et al. $(1988,1989)$ which resulted in the identification of major features in the the SCV site. The features were assumed to be planar fracture zones. First, a feature was identified in the tomograms. Then an estimate was made of the location of the intersections between the boreholes and the feature by identifying the feature as a reflector in the single-hole 
reflection analysis. This gives two possible orientatiors for the feature, one for each side of the cone intersecting the tomographic plane. If the feature is visible in both the N2-N3-N4 plane and the W1-W2 plane, then there is a further three-dimensional check on the geometry. Then the crosshole reflection data are also checked to see if a unique orientation can be choser ${ }_{i}$ for the zone.

Such analysis was performed separately for both the radar and the seismic data. Then all the data was integrated to obtain four major fracture zones: GA, GB, GH (subdivided into $\mathrm{GHa}$ and $\mathrm{GHb}$ ) and $\mathrm{GI}$ and a minor zone, $\mathrm{GC}$ as discussed in Olsson, et al. (1989). (n.b.: The " $G$ " in these names stands for geophysics. In the discussion following, we drop the " $G$ " when referring to hydrologic zones. Occasionally, we refer back to zones which were preliminarily identified only with radar data; these are preceded by a " $R$ ". The " $R$ " zones are not always located in the same place as the " $G$ " zones of the same name where both radar and seismic data was integrated into the description. However, the hydrologic zones are always in the same location as the " $G$ " zones.) Tavle 2.1 summarizes these results with a qualitative classification of the strength of the anomalies associated with the zones made in three classes: S, Strong; M, Medium; and W, Weak. Table 2.1 also includes zone B' which is discussed below.

Table 2.1. Geophysical Fracture Zones

\begin{tabular}{|cccccc|}
\hline Zone & Radar & Seismics & $\begin{array}{c}\text { Geoph } \\
\text { Logging }\end{array}$ & $\begin{array}{c}\text { Core } \\
\text { Logging }\end{array}$ & Hydrologic \\
\hline \hline A & S & S & M & M & W \\
B & S & S & M & M & M \\
B & S & S & M & W & S \\
C & M & S & W & W & W \\
H & S & S & M & S & S \\
I & M & W & M & M & S \\
\hline
\end{tabular}

\subsection{The Hydrologic Conceptual Model}

Figures 2.1a through e (after Olsson et al., 1988) give a summary of the borehole data acquired for the $\mathrm{N}$ - and $\mathrm{W}$-holes. The geophysical features identified by Olsson et. al. (1989) are shown on the plots as shaded horizontal bands. Under the hydraulic conductivity column on the right, we have blackened in those conductivities Treater than $10^{-8}$ $\mathrm{m} / \mathrm{s}$ in order to make the higher conductivity measurements stand out. If the geophysical features are taken as hydrologic zones with a width of about 10 meters (denoted by the "I" bars on the right hand sides of Figure 2.1), they account for about $60 \%$ of the measured hydraulic transmissivity measured in the boreholes (Table 1.2). Almost all of the remaining $40 \%$ of the transmissivity is accounted for in three zones: near 80 meters in borehole W2, 152 meters in N2 and from 80 to 90 meters borehole N4. There are strong radar and seismic anomalies at each of these locations as well as anomalies in the geophysical and core logs. 
W 1

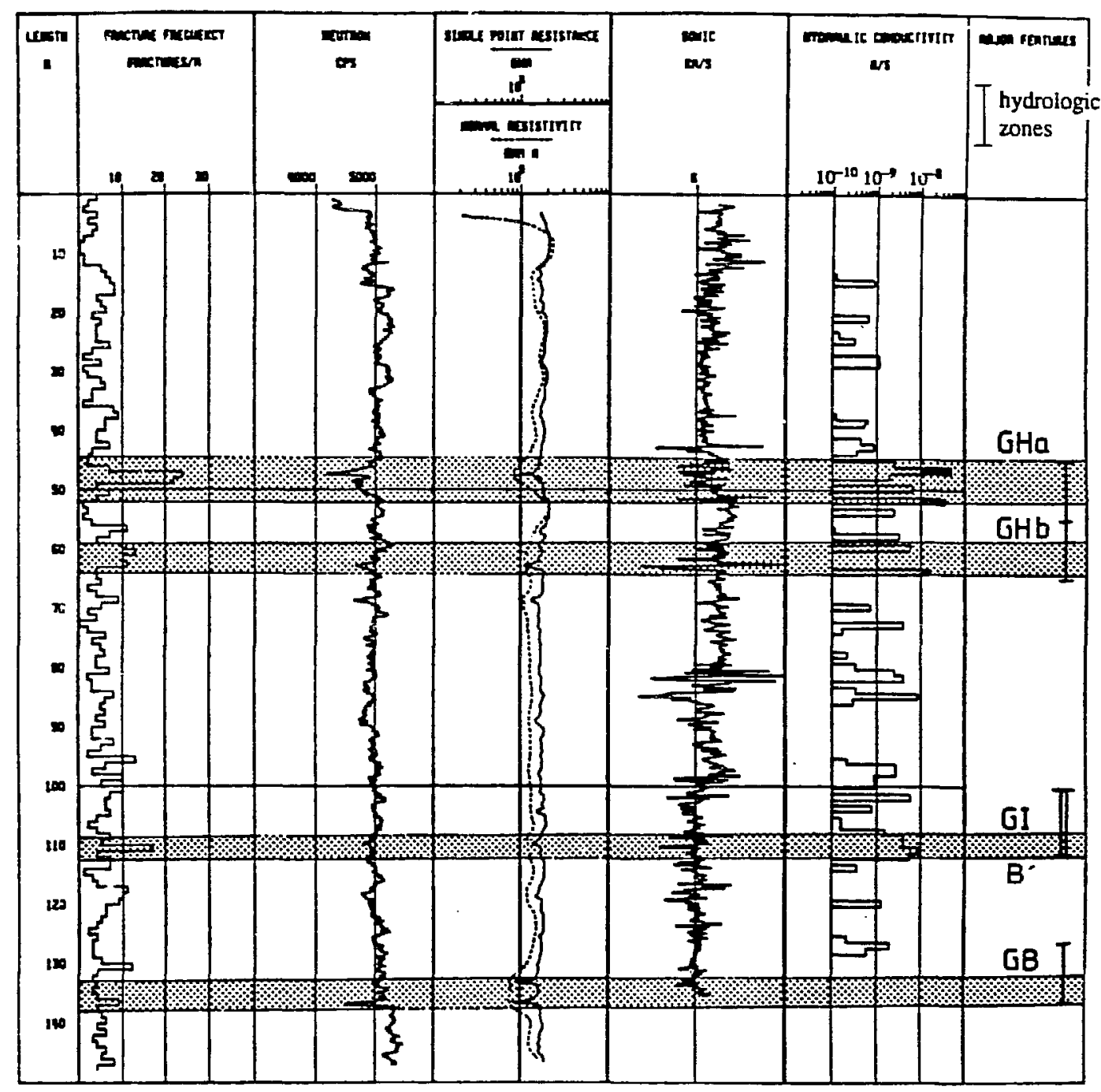

XBL $903-850$

Figure 2.1 a. Summary data sheet for W1. Hydraulic conductivities greater than $10^{-8} \mathrm{~m} / \mathrm{s}$ have been blackened in. Geophysical features are shown as shaded horizontal bands. Hydrologic zones are marked with "I" bars in the right hand column. 
W 2

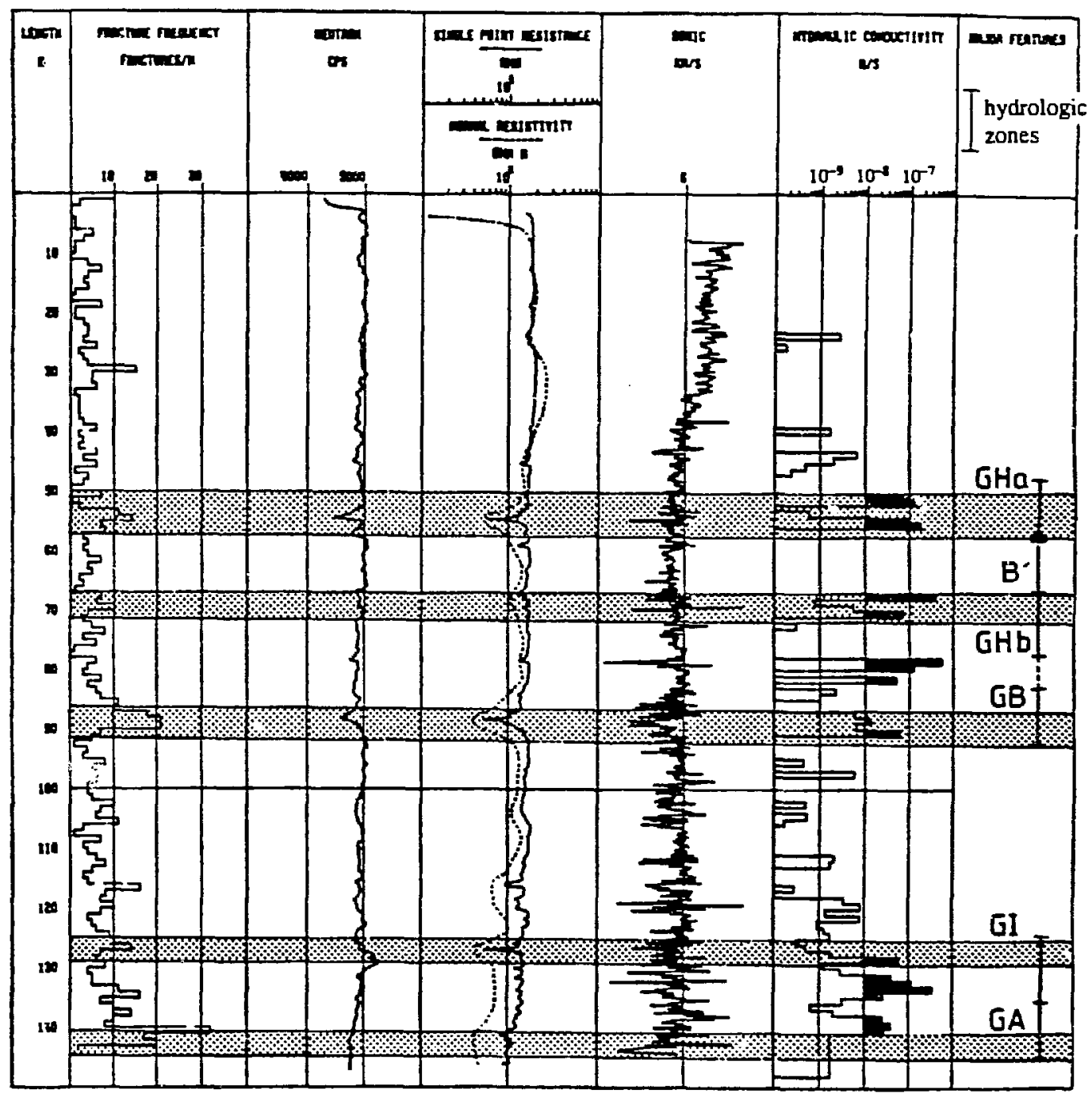

XBI 903-85I

Figure 2.1b. Summary data sheet for W2. Hydraulic conductivities greater than $10^{-8} \mathrm{~m} / \mathrm{s}$ have been blackened in. Geophysical features are shown as shaded horizontal bands. Hydrologic zones are marked with " $I$ " bars in the right hand column. 
N 2

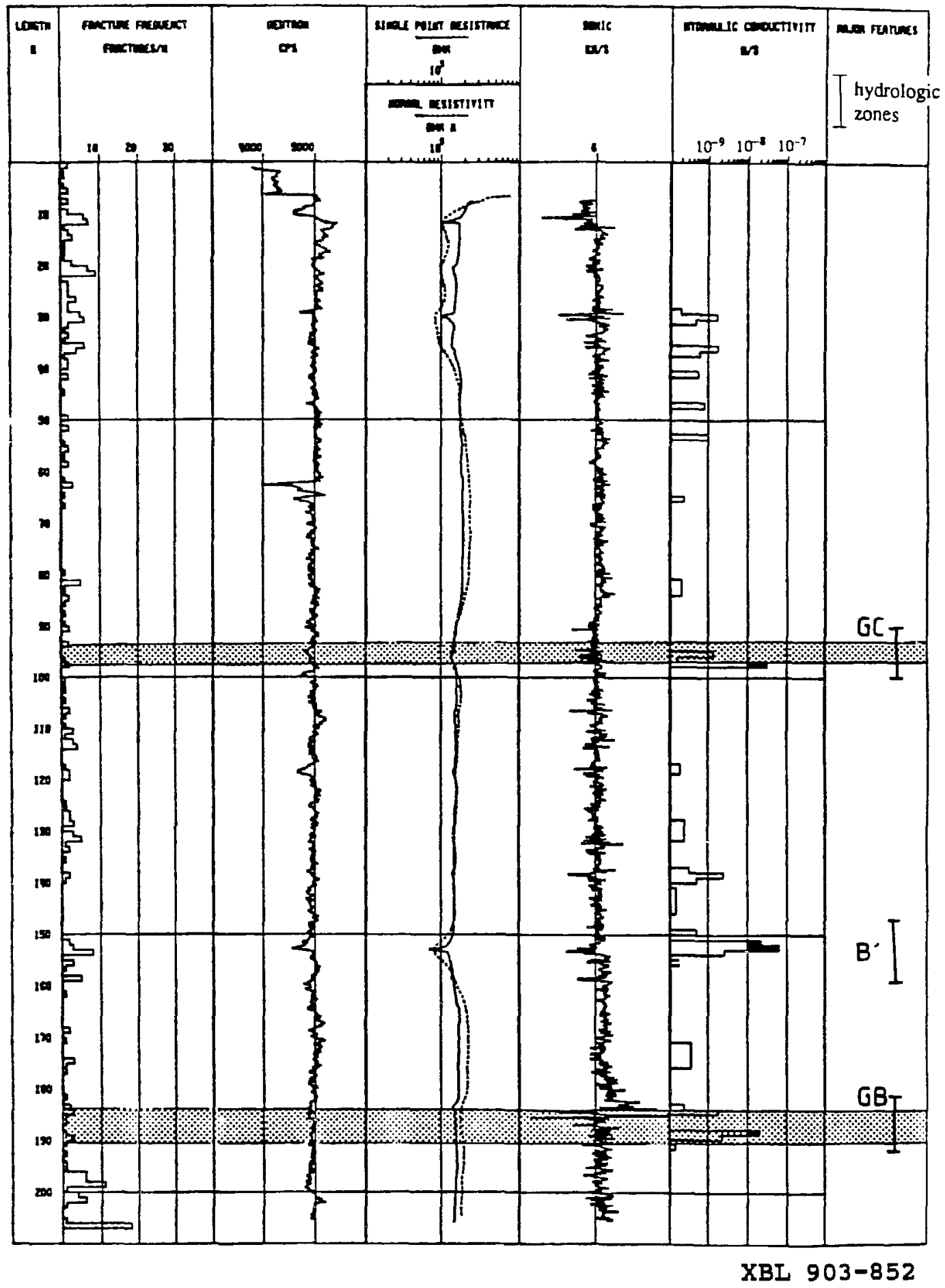

Figure 2.1c. Summary data sheet for N2. Hydraulic conductivities greater than $10^{-8} \mathrm{~m} / \mathrm{s}$ have been blackened in. Geophysical features are shown as shaded horizontal bands. Hydrologic zones are marked with "I" bars in the right hand column. 
N3

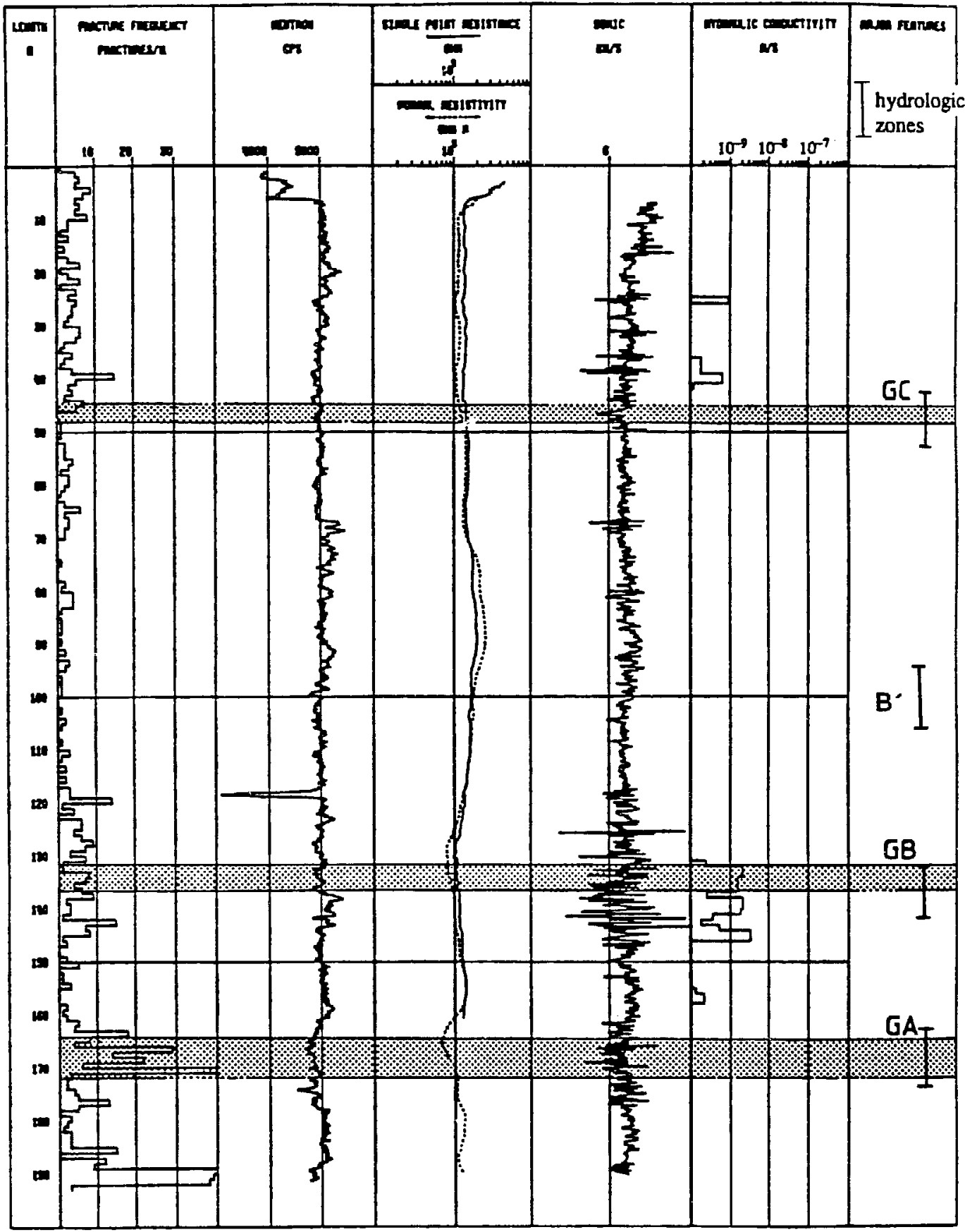

XBI, $903-853$

Figure 2.Id. Summary data sheet for N3. Hydraulic conductivities greater than $10^{-8} \mathrm{~m} / \mathrm{s}$ have been blackened in. Geophysical features are shown as shaded horizontal bands. Hydrologic zones are marked with "I" bars in the right hand column. 
N 4

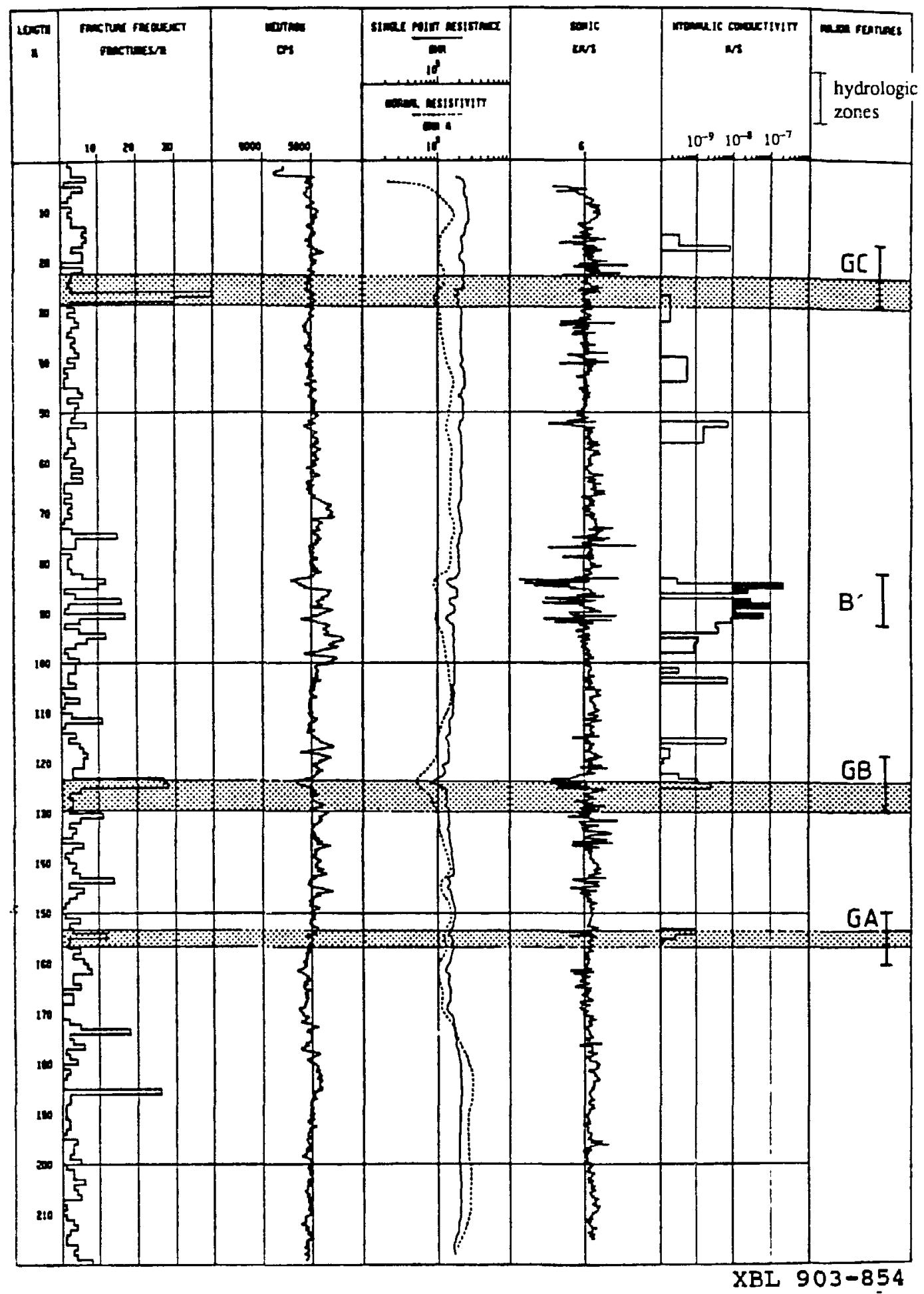

Figure 2.1e. Summary data sheet for N4. Hydraulic conductivities greater than $10^{-8} \mathrm{~m} / \mathrm{s}$ have been blackened in. Geophysical features are shown as shaded horizontal bands. Hydrologic zones are marked with "I" bars in the right hand column. 
Table 2.2. Hydraulic Transmissivity Distribution

\begin{tabular}{|lrrrrr|rr|}
\hline \multicolumn{7}{|c|}{ Hydraulic Transmissivity $\left(10^{-9}\right.$} & $\left.\mathrm{m}^{2} / \mathrm{s}\right)$ \\
\hline Zone & W1 & W2 & N2 & N3 & N4 & Total & \% of total \\
\hline \hline A & - & 99 & - & 0 & 1 & 100 & 3.2 \\
B & 0 & 88 & 20 & 12 & 5 & 125 & 4.0 \\
B & - & - & 80 & 0 & 450 & 530 & 17.1 \\
C & - & - & 36 & 0 & 0 & 36 & 1.1 \\
H & 120 & 950 & - & - & - & 1070 & 34.5 \\
I & 25 & 510 & - & - & - & 535 & 17.2 \\
(HB) & - & 670 & - & - & - & 670 & 21.6 \\
Sum & 145 & 2317 & 136 & 12 & 456 & 3066 & 98.7 \\
\hline \multicolumn{2}{l}{ Total transmissivity } & & & & 3100 & 100 \\
\hline
\end{tabular}

- Transmissivity between zones $\mathrm{H}$ and $\mathrm{B}$ in borehole W2

Initially, we tried to account for these hydrologic features by altering the orientations of the five major zones (A, B, C, H and I) an amount consistent with the precision of the geophysical analysis. After determining that no significant improvement was possible, another zone, B', was added to explain previously unaccounted for hydrologic anomalies in N4 and N2. Figure 2.2 shows a perspective plot where zones $B$ and $C$ are represented as dots located on planes. In this figure we are looking along the $B$ and $C$ planes so that the zones appear as dots clustered along a line. In this perspective, one can see that the hydrologic anomalies in $\mathrm{N} 4$ and $\mathrm{N} 2$ lie on a plane roughly half way between zone $B$ and zone $C$. For this reason, we chose $B$ ' to be a plane between $B$ and $C$ and parallel to the $A, B$, and $C$ zones. The addition of $B$ ' increases the percentage of transmissivity accounted for to about $78 \%$. The resulting hydrologic zone model is shown in Figure 2.3. in a perspective view from the North-Wesi.

The fact that no similar hydrologic anomaly where B' would intersect N3 (between N4 and $\mathrm{N}^{2}$ ) is not a problem for this model. This can easily be accounted for if $\mathrm{B}^{\prime}$ is impermeable in the vicinity of N3.

The B' zone also fits in well with some of the geophysical results, but because it did not fit well with all the results, it was not included in the final geophysical model. The radar results show a very strong low velocity zone in the section N4-N3 slowness tomograms corresponding to the feature at 90 meters down borehole N4 (Figure 2.4, from Olsson et al., 1989) as well as single hole radar reflectors on either side. On Figure 2.4 our B' feature is located at the dark area near N4 labeled "RB' ( $R$ for radar). This is because the original rajar interpretation put zone $B$ in this location. (Later, the integration of radar and seismic results put zone $G B$ ( $G$ for geophysics) up to just below where zone RA is on Figure 2.4.) This tomogram shows the B' (or RB) anomaly begins to peter out about 20 meters from the borehole. Also, several strong reflectors are observed near the region 152 meters down borehole N2. On the other hand, the B' feature is not apparent in any of the attenuation tomograms or the section N2-N4 slowness tomograms, which is why it was not included in the final model. The reason for this absence of B' in the attenuation tomograms is not apparent. However, the N4-N3 tomogram where B' does show up is thought to be more accurate than the N2-N4 tomogram where it does not (O. Olsson, personal communication). 


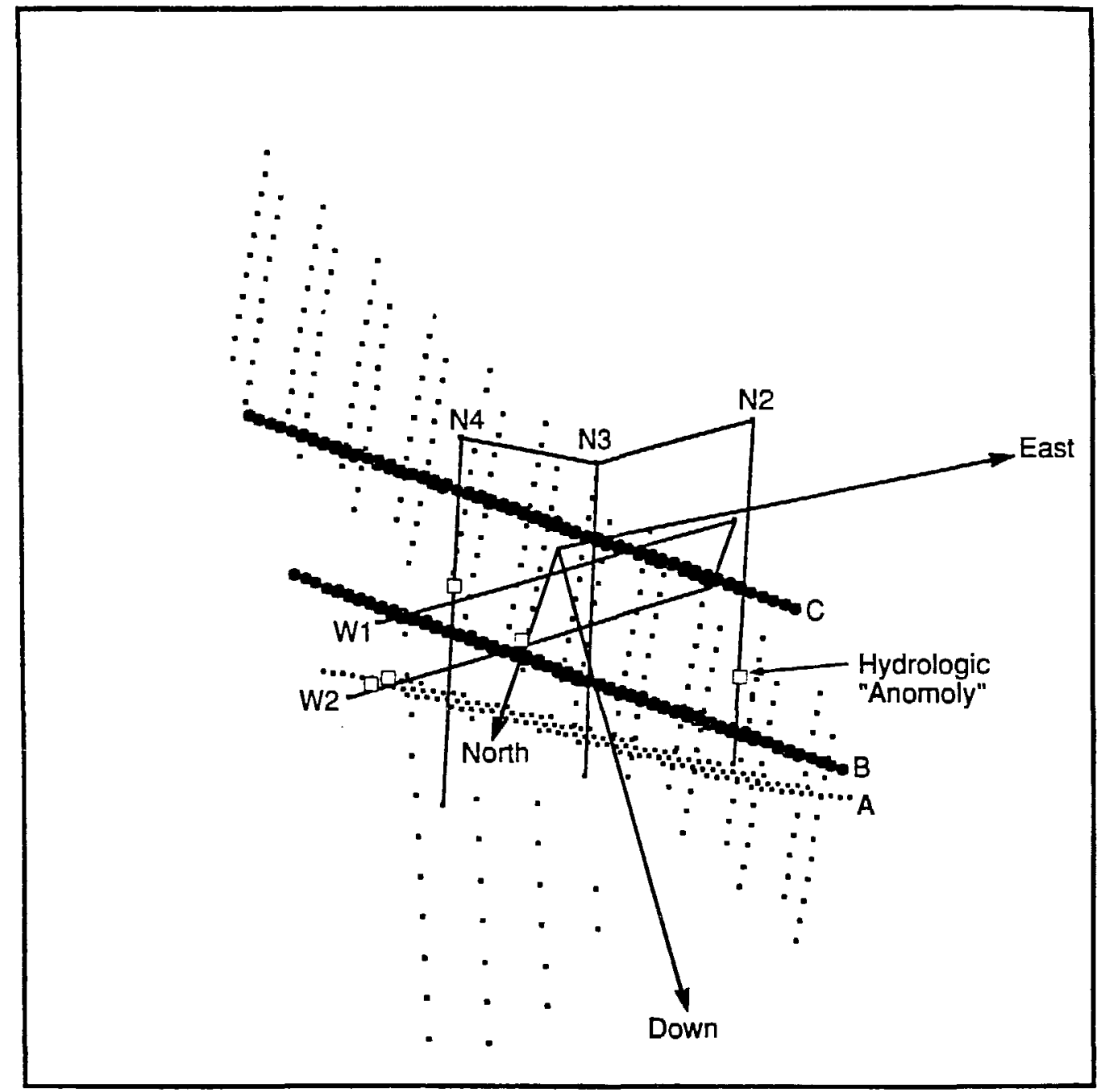

XBL 891.6143

Figure 2.2. A perspective view of the SCV block looking up to the North-East showing zones $B$ and $C$ and two hydrologic anomalies that lie between these zones in a parallel plane. 


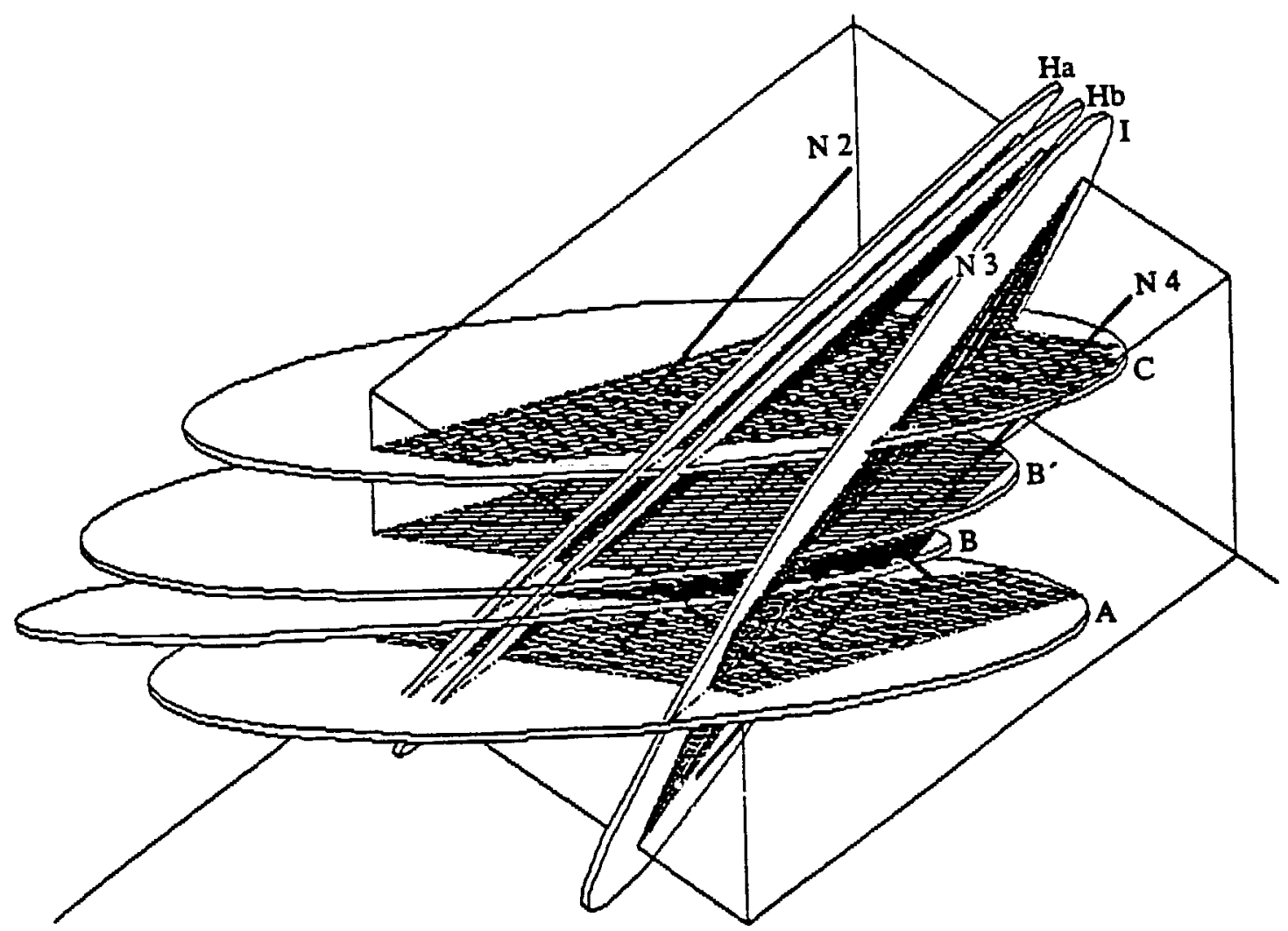

XBL 896-2396

Figure 2.3. The hydrologic zone model shown in perspective from the Norh-West looking down. Zones A, B, B', C, Ha, Hb and I are shown. Gridding on the planes represents the hydraulic conductors of the template used for annealing. 

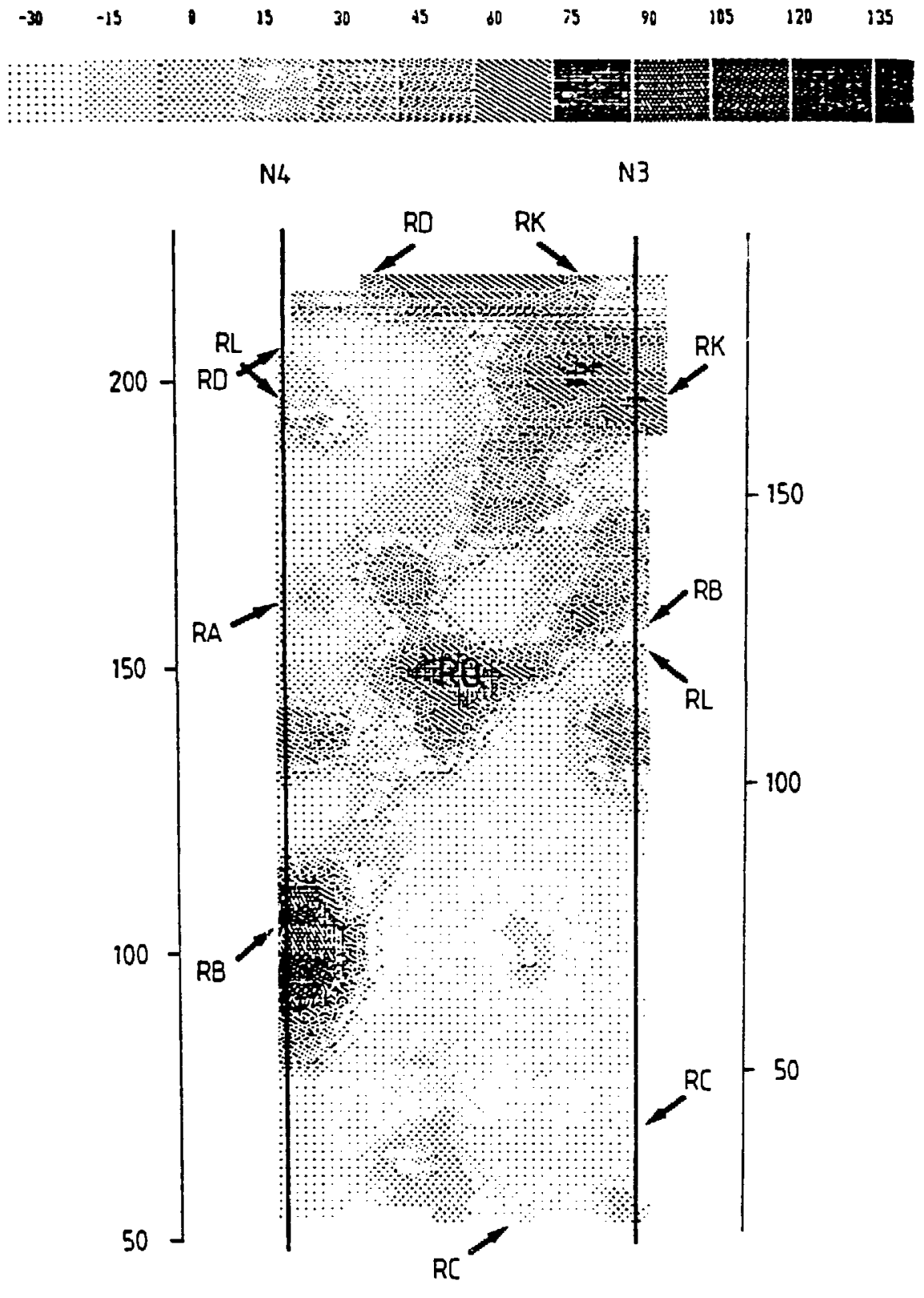

XBI 896-2397

Figure 2.4. Residual radar slowness tomogram for the borehole section N3-N4 made with a center frequency of $22 \mathrm{MHz}$. 
The B' feature may be related to the RQ feature shown on Figure 2.2. It appears that B' may intersect the South edge of the $R Q$ feature. The feature GB then skirts the North edge of $R Q$ and produces the largest hydrologic anomaly in N3. It may be that $R Q$ is a step between en echelon fracture zones represented by $B^{\prime}$ and GB. This could also explain the lack of hydrologic activity in N3 at B'. However, it does not explain why $B^{\prime}$ is again the largest anomaly in N2 unless there is another step in the opposite direction.

One remaining hydrologic anomaly in W2 between zones $\mathrm{H}$ and $\mathrm{B}$ (called " $\mathrm{HB}$ " in Table 1.2) accounts for $21.7 \%$ of the transmissivity. If we allocate this transmissivity party to $\mathrm{H}$ and partly to $\mathrm{B}$, then we can account for $98.7 \%$ of the observed transmissivity with a zone model. This makes a certain amount of sense when we consider that the transmissivity measured in the boreholes is not strictly additive. Figure 2.5 illustrates that successive borehole to ${ }^{-} t e$ actually sampling the some of the same transn -ivity. It is easy to imagine wla. the high conductivity found between zones $H$ and $\mathrm{B}$ is $\mathrm{W} 2$ is due to a few conductive features that are related to $\mathrm{H}$ and $\mathrm{B}$ and possibly related to the intersection of $\mathrm{H}$ and $\mathrm{B}$.

The wnes in the hydrologic model are represented as disc-shaped planes. As we do not expect the zones to be unifo mly permeable, the zones are discretized into flow channels. Any type of discretization could be used. We base the choice of grid with the suppor of geomechanical investigations of the shear zones explained below. Then, as explained in Chapter 4, we use annealing to find a configuration of active grid elements which can reproduce the observed hydrologic behavior of the system.

\subsection{Geomechanica! interpretation of the Shear Zones}

Slickenside striations in the two sets of fracture zones indicate that they have undergone shear deformation. Utilizing a database consisting of 3100 logged fractures from borehole core and 900 fractures mapped on the walls of underground drifts, the character of these fracture zones have been analyzed. This data along with numerical fracture mechanics modeling suggest that the higher fluid conductivities in the zones compared with the surrounding rock may be due to a combination of higher fracture densities, and stresses in the zones that promote dilatancy in fractures with certain orientations. This would also result in anisotropic flow in the zones, with the primary flow direction in the direction of the dilatant fractures.

The $\mathbf{H}$ and I fracture zones strike roughly north-south and dip steeply to the east, and the A, B, B', and C zones strike northeast and dip 35 to $40^{\circ}$ to the southeast (Olsson et al., 1988). Core logs from these boreholes show that the zones vary in width from 2 to $15 \mathrm{~m}$, and have a higher fracture density than the surrounding ground (fracture density in the zones varies from 10 to 30 fractures $/ \mathrm{m}$, compared with 1 to 20 fractures $/ \mathrm{m}$ in the ground surrounding the zones). The borehole data base contains information on 3100 fractures of known orientation. The five boreholes (N2, N3, N4, W1, W2) intersect the fracture zones in over 20 locations. Scanline surveys were taken in the drifts surrounding the SCV site, which contain photographs, orientations, and associated information for 900 mapped fractures. In addition, a few detailed wall maps were made along some of the underground drifts. This information is used in the context of the overall geological framework of the area surrounding the Stripa mine, and stress measurements indicating the present stress state (Chan et al., 1981; Carlsten, 1985; Olsson et al., 1988).

Based on an analysis of this data, there is evidence that the fracture zones in the SCV block have undergone extensive shear deformation. Slickensided fractures in the zones indicate that these zones contain faults. Also, where these zones intersect underground 


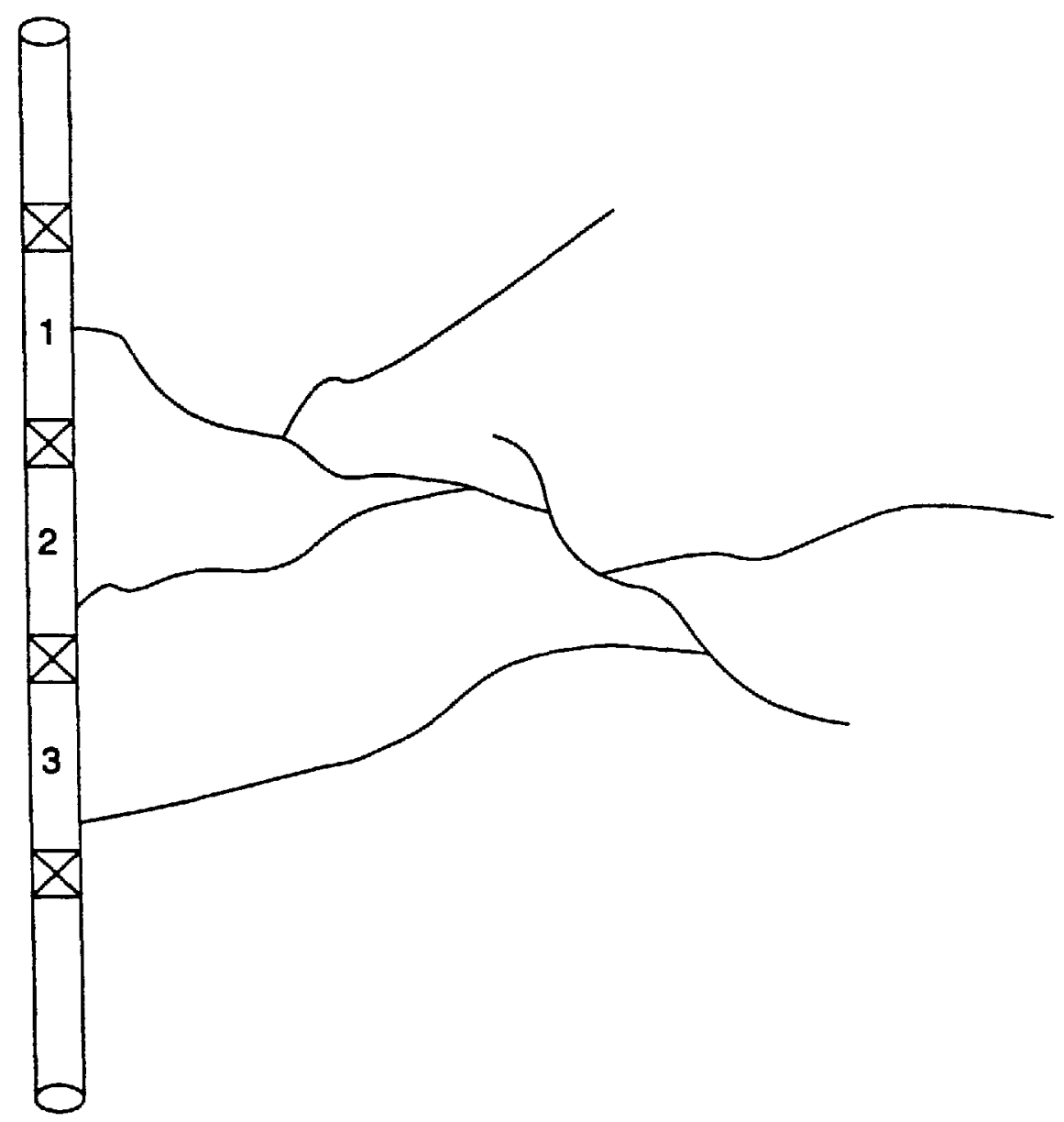

XBI 903-855

Figure 2.5. Hypothetical testing zones in a fractured rock showing that the transmissivity measured in the three zones will overlap such that the transmissivity measured separately will add up to more transmissivity than the true total. 
drifts, cracks that splay from slickensided fractures can be observed. We infer that the zones identified by radar and seismic tomography are mostly fault zones. Certain pattems of fracturing can be expected in fault zones in brittle rock (see Section 2.3.2 below). and some of these pattems have been observed in the SCV block. This data, along with numerical modeling suggest that the higher fluid conductivities in the zones compared with the surrounding rock may be due to a combination of higher fracture densities, and stresses in the zones that promote dilatancy in fractures with cerain orientations.

Laboratory tests of intact rock under direct shear by Tchalenko (1970) and others indicate that initially a set of en echelon fractures form, finally linking up into a throughgoing fault. Shear fractures formed in this way can show a remarkable resemblance to faults mapped in the field (Tchalenko, 1970; Sibson, 1986). On the other hand, the field studies of Segall and Pollard (1983), Martel et al. (1988), and others indicate that faults can form from slip along pre-existing joints. As slip increases along these pre-existing fractures, secondary fractures form and link the slipping fractures to form throughgoing faults.

In both of these scenarios, a high density of fracturing develops adjacent to the throughgoing slip surfaces. These associated fractures can be subjected to stress states much different than fractures in the surrounding rock. For instance, in-situ stresses in the ground are normally compressive in all three principal direction. However, even under compressive in-situ stresses, slip along fractures can locally induce tensional stresses (Rodgers, 1980; Segall and Pollard, 1980; Sibson, 1986). Fractures that open in these extensional regions can have hydraulic conductivities much greater than fractures in surrounding areas which are under compressive stresses in all directions. Mineralized areas commonly occur in regions such as these (Sibson, 1981). Also, extensional fractures in shear zones and faults will have a preferred orientation, and this can result in an anisotropic flow regime in the shear zone. Thus the orientation and density of fractures in a rock mass is not sufficient to determine flow properties. It is also necessary to consider the presence of shear zones or faults, and the overall in-situ stress state.

\subsubsection{Fractures in the SCV Block}

Before looking at the fracture zones themselves, we give a general overview of the pattem of fracturing in the SCV block. The granite hosting the fracture zones at the SCV site is fractured itself, albeit not as extensively as the fracture zones. Over 3000 fractures are intersected by the five boreholes in the SCV block. A statistical analysis of the orientations and locations of these fractures is given in Olsson et al. (1988). When fracture data from all five boreholes are combined, three main fracture orientation clusters are observed. Two of these roughly coincide with the orientations for the fracture zones shown in Figure 2.3, and the third cluster group is sub-horizontal. The subhorizontal fractures represent a small percentage of the total number of logged fractures, but this is partly due to the low plunge of the boreholes drilled through the SCV block. Next, we focus on the fracture zones.

The NE-striking, low dipping fracture zones includes $A, B, B$, and $C$. We focus on zone $B^{\prime}$, since it represents a highly conductive feature in the SCV block. Poles to the fractures in zone $B^{\prime}$ where it intersects borehole N4 (depths 80 to $95 \mathrm{~m}$ ) are shown in Figure 2.6. The dark square in Figure 2.6 represents the best estimate for the orientation of this zone, based on where it intersects boreholes and as described above. Some of the fractures in this zone are slickensided, and these fractures are marked with hollow squares in Figure 2.6. Figure 2.6 shows that most of the slickensided fractures and 


\section{Zone GB'}

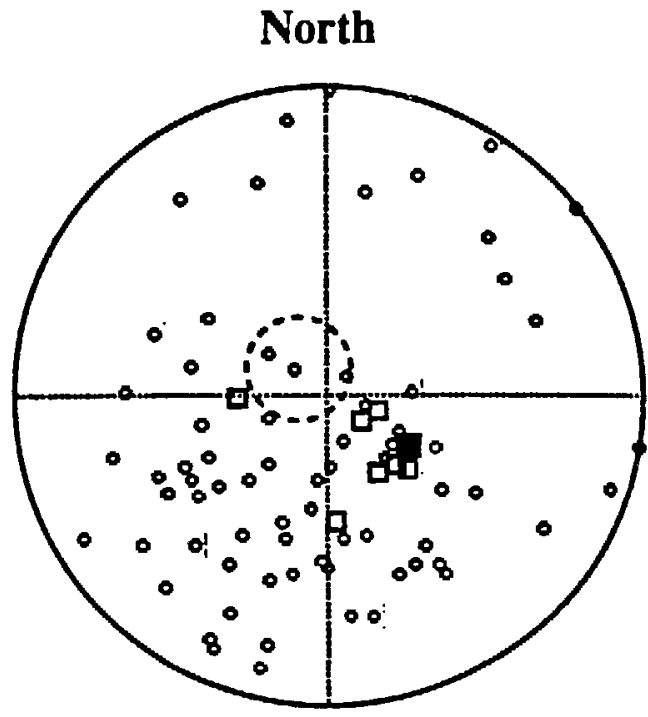

XBL $903-857$

Figure 2.6. Poles to the fractures in zone GB' (upper hemisphere) where it intersects borehole N4 (depths $80-95 \mathrm{~m}$ ).
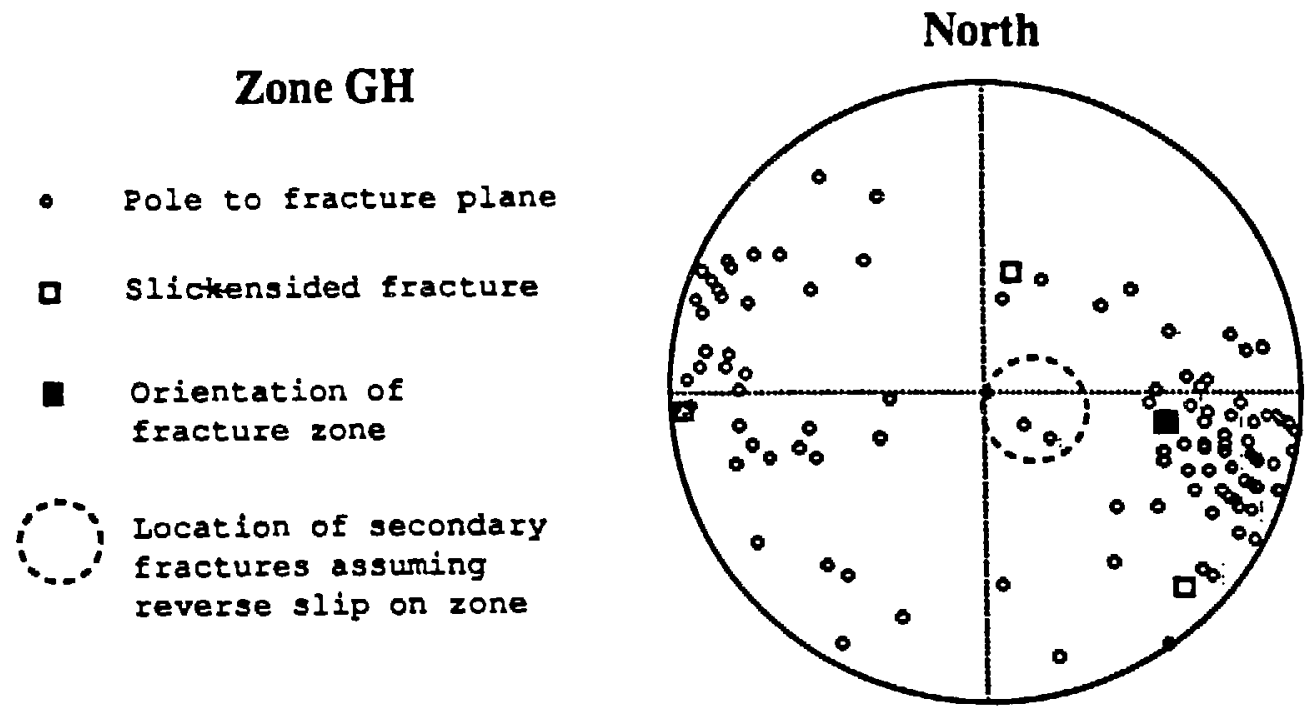

XBL $903-856$

Figure 2.7. Poles to the fractures in zone $\mathrm{GH}$ (upper hemisphere where it intersects borehole W1 (depths $48-63 \mathrm{~m}$ ). 
many of the non-slickensided fractures are sub-parallel to the orientation of the zone. We infer that the slickensided fractures represent major faults that parallel the zone as 3 whole. Steeply dipping striae on slickensided fractures that are sub-parallel to the zone indicate a dip-slip type of motion for the northeast striking, low dipping zones. Sub-horizontal cracks that splay from some of these slickensided fractures can be seen on the drift walls and indicate a reverse dip-slip motion (Petit, 1987). This sense of motion is consistent with stress measurements in the SCV site, indicating a present maximum horizontal compression oriented northwest (Chan et al., 1981; Olsson et al., 1988).

Many, if not most of the fractures logged where zone B' intersects borehole N4 may not be associated with the shear zone, but may instead represent "background" fractures in the host rock (as described in the previous section). Certainly the fractures that are sub-parallel to the zone are more likely to be related to the zone. Also, secondary extensile fractures may be associated with slip across the zone. Secondary extensile fractures are apparent in the driftwalls, but are difficult to identify from borehole logs. These dilatant fractures should have a narrow range of orientations, related to the orientation of the zone, the direction of slip in the zone, and the orientation of the far field stresses (e.g., Petit, 1987). For the northeast-striking, low dipping zones under reverse dip-slip motion, these fractures should plot within the circle shown in Figure 2.6. Thus fractures within this circle may be extensile fractures that are associated with reverse-slip on this zone. Also, fractures with this orientation may be contributing more to the overall conductivity of the zone than the fractures that are sub-parallel to the zone. For instance, Teufel (1987) shows that due to the accumulation of gouge, the permeability of fractures under shear in the laboratory decreases with increasing shear displacement. Thus the slickenside fractures may not be contributing substantially to the conductivity of the zone. Teufel (1987) notes, however, that the rock immediately adjacent to the sheared fracture may be highly conductive due to extensile microfracturing formed from the shearing.

Fractures within the circle in Figure 2.6 are sub-horizontal. Based on this, we infer that sub-horizontal fractures would be preferred pathways for flow within the NE-striking zones in the SCV block. However, in Figure 2.6 only a few fractures occur with this orientation. This may be partiy due to the bias against sub-horizontal fractures appearing in a borehole that plunges only $18.6^{\circ}$. Sub-horizontal fractures have been mapped in the driftwalls surrouriding the SCV block, and some of these fractures have been identified as secondary fractures due to slip in the NE-striking zones.

The N-S striking, steeply dipping zones includes $\mathrm{H}$ (Ha plus $\mathrm{Hb}$ ), and I. We focus on the $\mathrm{H}$ zone, since it is the most conductive feature in the SCV block. Poles to the fractures in zone $H$ where it intersects borehole Wl (depths 48 to $63 \mathrm{~m}$ ) are shown in Figure 2.7. Steeply dipping striae on slickensided fractures, along with shallowly-dipping splays suggest reverse dip-slip movement along zone $H$. Secondary fractures due to reverse slip on this zone should strike N-S and dip $10-40^{\circ}$ to the east, as shown in the circle in Figure 2.7 . Because boreholes $W 1$ and $W 2$ dip only $5^{\circ}$, there will be a bias in the boreholes against fractures with this orientation. However, shallowly dipping fractures associated with zone $\mathrm{H}$ are seen in the driftwalls. We infer that shallowly dipping (to the east), N-S striking fractures would be preferred pathways for flow in these zones.

\subsubsection{Geomechanical Modeling of the Shear Zones}

The possible high conductivity of secondary fractures in the zones, compared with slickensided fractures in the zones and fractures outside the zones is supported by the 
results of the numerical example shown in Figure 2.8. We have considered a simplified two dimensional elastic model under far field compressive horizontal and verical stresses $\sigma_{1}$ and $\sigma_{2}$. According to Chan et al. (1981) and others, the maximum principal in-situ stress is horizontal and has a magnitude of approximately $24 \mathrm{MPa}$ on tie $360 \mathrm{~m}$ level. This stress is oriented northwest and is therefore perpendicular to the NE-striking zones. The minimum principal stress is vertical and has a magnitude of approximately $9 \mathrm{MPa}$ on the $360 \mathrm{~m}$ level. In Figure 2.8a, nine randomly distributed fractures are subjected to these stresses. For simplicity it is assumed that the fractures do not cross each other, and each of these fractures have been given a length of one meter. In Figure $2.8 \mathrm{~b}$ we look at the same system of fractures, except now two long fractures representing the throughgoing slickensided fractures are added that dip $40^{\circ}$. The long fractures each have a length of $16 \mathrm{~m}$ and are separated by $7 \mathrm{~m}$. Thus in Figure $2.8 \mathrm{~b}$ the nine fractures are subjected to the stress state within one of the NE-striking, low dipping zones i.e., the A, B, B' and C zones.

Due to the application of the in-situ stresses, the modes I and II crack-tip stress intensity factors, $K_{l}$ and $K_{I J}$, respectively, have been calculated at each of the crack tips utilizing a numerical algorithm based on the work of Kachanov (1987). This algorithm has been modified to account for cracks that are closed by the compressive stresses but can still shear (frictionless surfaces are assumed for closed cracks). The $K_{I}$ and $K_{I I}$ for each crack is presented in the table below Figure 2.8. Each crack has two crack tips, and in Figure 2.8 we only show the average of the two crack tips. $K_{I}$ is an indication of the opening of the crack, and $K_{l l}$ is an indication of the shearing of the crack (Lawn and Wilshaw, 1975). $K_{I /}$ can have both negative and positive signs indicating shear in one direction or the other, while $K_{l}$ can be either positive or zero. $K_{I}=0$ indicates that the crack is closed.

Figures $2.8 \mathrm{a}$ and $4 \mathrm{~b}$ demonstrate the importance of shear zones in localizing flow in the rock mass. In Figure 2.8a, each of the fractures are under different amounts of shear but they are all closed $\left(K_{I}=0\right)$. In Figure $2.8 \mathrm{~b}$, however, due to the localization of shear from the long slickensided fractures, fractures with certain orientations have a positive $K_{I}$, and are therefore open. These fractures are sub-horizontal, which agrees with the expected orientations as shown in Figures 2.5 and 2.6.

Figure 2.8 also gives the stress intensity factors for the long fractures, and they remain closed. The sign of $K_{I I}$ for the long fractures indicates a reverse dip-slip motion, as the field data suggests for the NE-striking zones. Thus this numerical example suppors the idea that the conductivity is greater in dilatant fractures in the zones rather than the main throughgoing fractures that are sub-parallel to the zone.

Figure 2.8 also points out a technical difference between a fault zone and a fracture zone. A fracture zone may contain a high density of fractures, but under compressive far field stresses, the fractures will be closed, as in Figure 2.8a. A fault zone, on the other hand, by containing dilatant fractures, can be more conductive, even if the density of fractures is lower.

\subsection{Conclusions}

Seismic and radar tomography of the SCV underground test area in Stripa, Sweden have detected two major sets of fracture zones. We have evidence that they are fault zones under reverse dip-slip motion. Associated with slip in the zones, secondary fracturing has been observed. For the NE-striking. low dipping zones, the secondary fractures are sub-horizontal, and for the N-S striking, steeply dipping zones, the secondary fractures strike $N-S$ and dip $10-40^{\circ}$ to the east. Numerical modeling indicates that 


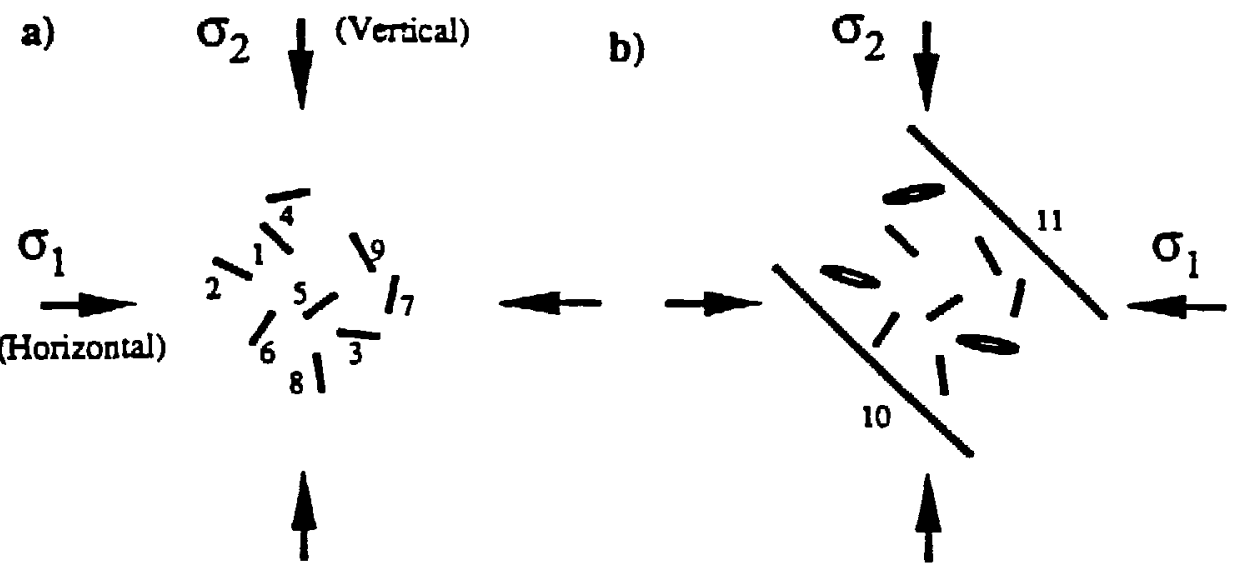

a)

\begin{tabular}{lllr}
\hline$\#$ & $\mathrm{~K}_{1}$ & $(\mathrm{MPa} \sqrt{\mathrm{m}})$ & $\mathrm{K}_{\mathrm{II}}$ \\
1 & 0 & & -7.8 \\
2 & 0 & -4.4 \\
3 & 0 & 3.7 \\
4 & 0 & 5.4 \\
5 & 0 & 8.1 \\
6 & 0 & 5.9 \\
7 & 0 & 2.3 \\
8 & 0 & -4.4 \\
9 & 0 & -7.5 \\
10 & 0 & - \\
11 & 0 & -
\end{tabular}

b)

\begin{tabular}{cllr}
$*$ & $\mathrm{~K}_{1}$ & $(\mathrm{MPa} \sqrt{\mathrm{m}})$ & \multicolumn{1}{c}{$\mathrm{K}_{\mathbf{I}}$} \\
1 & 0 & & -5.3 \\
2 & 0.92 & -4.5 \\
3 & 0.55 & 0.4 \\
4 & 0.05 & 7.8 \\
5 & 0 & 12.9 \\
6 & 0 & 15.5 \\
7 & 0 & 12.4 \\
8 & 0 & 5.3 \\
9 & 0 & -0.6 \\
10 & 0 & -38.6 \\
11 & 0 & -38.6
\end{tabular}

XBL $896-2393$

Figure 2.8. (a) Nine randomly distributed fractures subjected to the maximum horizontal and vertical stresses on the $360 \mathrm{~m}$ level; (b) Two long fractures representing the slickensided fractures in the NE-striking zones are added. The table on the figure lists average Modes I and II stress intensity factors for each crack for the two cases. 
3.0. THE NUMERICAL MODEL

\subsection{The CHANGE Model}

The channel network generator CHANGE (Billaux, et al., 1988) is used to construct a finite element mesh for the hydrologic conceptual model. CHANGE can generate a stochastic network of conductors or any regular grid of conductors. In the finite element analysis, each element is assumed to represent a one-dimensional conductor, i.e. a pipe. For the purpose of calculating flow, we do not need to describe the shape of the conductor. We only need to know the location of the endpoints and the conductance of the element which has the units of permeability times cross-sectional area: $\mathrm{m}^{3} / \mathrm{s}$. An element is not necessarily meant to precisely represent a specific channel. It may represent the equivalent conductance of several channels combined.

\subsection{TRINET}

TRINET (Karasaki, 1988) is used to calculate the hydrologic response of the zone model to the simulated well test. TRLNET is a finite element code for simulating fluid flow and advective and dispersive solute transport in 3-dimensional networks of onedimensional conductors. The code incorporates a mixed Lagrangian and Eulerian scheme with adaptive gridding for transport calculations. The model avoids numerical dispersion by creating new Eulerian grid points instead of interpolating the advected profile back to the fixed Eulerian grid.

The code first solves the flow field using a simple Galerkin finite element method. The flow can be either steady-state or transient, where the time derivative is treated in a usual finite difference manner. From the pressure distribution at a given time the velocity distribution in the fracture network is calculated. Since linear shape functions are used to solve the flow field, the velocity is uniform within a given element. Elements are sub-divided as necessary for transient calculations. The advection-dispersion equation for mass concentration is then solved by decoupling the equation into two stages. In this way it is possible to solve the advection term independently of the diffusion term and minimize the numerical dispersion.

First, the advection equation is solved by using the method of characteristics. The concentration profile at the end of the calculation in the previous time step is, in effect, the initial-value distribution for the new advection problem. This profile is advected explicitly in the Lagrangian manner according to the velocity in each element. The advected front is placed on the Eulerian grid and new nodal points are generated to preserve the exact shape of the front. At every time step the element catalog is revised and the nodal points are renumbered to keep the band-width minimized. Because it is necessary to preserve the geometry of the fracture network itself, the original nodal points must be kept intact. The method of single-step backward particle tracking or reverse streaklines is used to obtain the concentration values for these fixed nodal points.

The new concentration profile at the end of the advection stage is now the initial-value for the dispersion calculation in the second stage. The dispersion is treated in the usual finite element manner.

In the present study, only the flow is simulated using TRDNET. As data become 
available, however, TRINET will be used to simulate tracer transport. In this case, we need to know more about the structure of the conductance elements. For the purpose of flow, it does not matter whether we have a high conductivity channel with a small cross-sectional area or vice versa. For transport, the fluid velocity through the high conductivity channel with a small cross-sectional area will be larger than for the opposite case. This means, not surprisingly, that if we construct a model that predicts flow, we do not have enough information to predict transport. Independent information would have to be used to relate flux to velocity in each element.

\subsection{Scale of Analysis ("Resolution")}

In modeling the hydrology at the SCV site, we opted to use a channel network model as was described earlier. Thus, the system will be represented by a finite number of elements. It is now necessary to determine the scale of analysis, i.e., how finely we will discretize each zone. The scale of analysis is constrained by the size of the physical memory of the computer. This effectively sets the maximum number of conductors that can be modeled which in tum determines the maximum resolution. With the current configuration of the computer at LBL, the maximum number of nodes that is feasible for annealing process is about 10,000 . This is roughly equivalent to having a 10 meter grid spacing on each fracture zone.

Another factor that affects the scale of analysis is the density of the monitoring points. The minimum spacing of the conductors should be less than that of the monitoring points. One would like to have more than a single conductor between two monitoring points to reconstruct the geometry of the fracture zone. This effectively sets the minimum size of the mesh. It is in general desirable to have as many monitoring points as possible to accurately characterize a site. In the case of SCV site, the average spacing of monitoring points is on the order of a few to several tens of meters. The grid spacing needs to be smaller than the distance between monitoring points, which again gives approximately 10 meters as the appropriate grid spacing.

Finally, the purpose of our simulation is to resolve an equivalent hydrologic geometry of the fracture zones and not individual fractures. It is known that the drawdown effect due to open W2 is felt all across the site to R-holes at the ventilation drift. The scale of the fracture zones, therefore, is estimated to be on the order of several tens of meters to a few hundred meters in extent. From the above point of view, a grid spacing of about 10 meters is again reasonable. In onder to divide the plane by an even number, the pacing that was actually used placed a gird of $8.8 \mathrm{~m}$ squares on each plane.

\subsection{Treatment of the D-Holes as One Big Hole}

The current resolution of the analysis is on the order of 10 meters, but the five D-holes are only about 0.1 meters in diameter and 1.2 meters apart. In predicting the inflow into the D-holes, therefore, they have to be treated as one big hole.

Because the background permeability is neglected, the predicted inflow into the drift will be zero except where the zones intersect the drift. This may be a good assumption based on the fact that the background permeability is a very small percentage of the zone permeability. One can find analogous cases in any one of the $\mathrm{N}$ and $\mathrm{W}$ holes, where nearly all of the transmissivity is localized in a few zones.

\subsection{Connecting the Zones to the Boreholes}

As the boreholes in general are about 0.1 meters in diameter, it is extremely unlikely 
that any of the boreholes will intersect a channel in the zones. To insure a hydraulic connection between the zones and the wells, small "fins" were added to the boreholes at the points where they intersect the zones. These fins are made of two orthogonal discs 6 meters in diameter. The construction of the fins allowed the intersections between the fin discs to be channels which in turn intersect zone channels thus becoming connections to the borehole. The fins and zones are shown in Figure 3.1. 


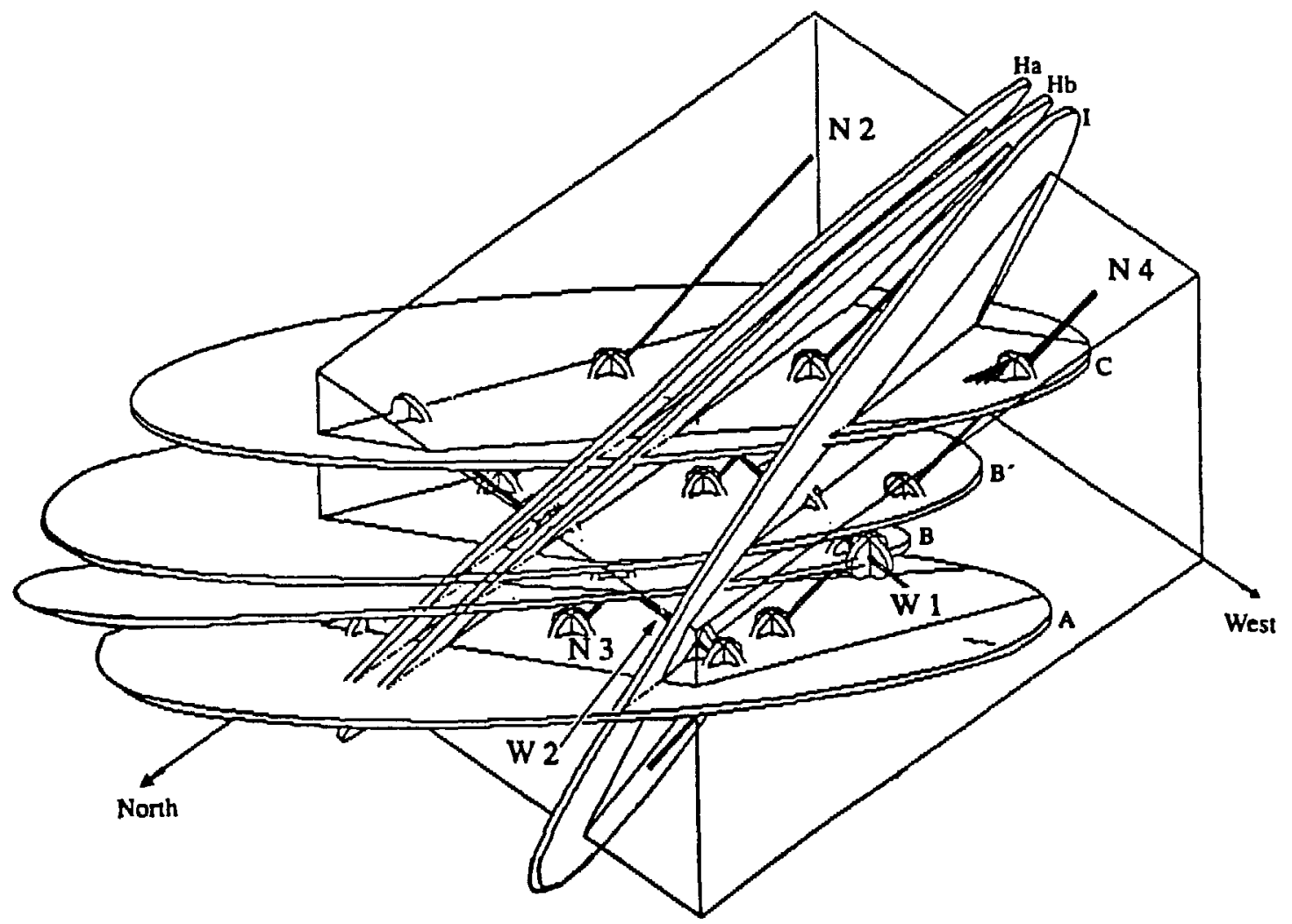

XBL $903-858$

Figure 3.1. The hydrologic zone model with the fins that connect the zones to the boreholes. 


\subsection{SIMULATED ANNEALING}

LBL has been developing an inversion technique called "Simulated Annealing" which can be used to construct a system which is functionally equivalent to the observed system: i.e., a model which simulates the same behavior as the observations we have. We describe here how to use annealing to find an equivalent fracture network model. The fracture network model is "annealed" by continually modifying the base model, or "template" such that the modified systems behave more and more like the observed system.

Hydrologic inversion models developed in the past, such as the conjugate gradient method, or maximum likelihood method (Carrera and Neuman, 1986) were focussed on detemining the conductivity values when the pattem of conductors is known or when everything is well interconnected as in the porous medium case. Annealing could theoretically be used to do this type of inversion, but might be relatively inefficient in this role. On the other hand, these porous medium techniques work poorly when one needs to completely tum off the conductivity of a portion of the region. Thus they are not the technique of choice for fracture systems when we wish to determine how the conductive features are connected.

Annealing is specifically designed to determine an appropriate pattem of conductors among a set of possible configurations. In fracture hydrology, we think that the pattem of conductors is responsible for the first order behavior. In other words, it is most important to know how the system is connected. Annealing is designed to find connections equivalent to those in the field. This is achieved by methodically searching pattems to see which ones behave like the ones observed in the field. Simulated annealing is actually the statistical technique which controls the acceptance or rejection of trial modifications. The job of simulated annealing is to find near optimal solutions of complicated function with many possible solutions and local minima.

The set of possible configurations is based on a template or base model which specifies all of the possible connectors. We are pursuing two different approaches to defining the template. The first approach has been described above. We make the assumption that the behavior is controlled by fracture zones, so we only allow connectors to exist within the zones. This approach has the advantage that it is efficient because it uses the information gained from geophysics and geology a priori. The second approach is to use a three-dimensional regular grid of conductors as the initial template. This approach is described in Appendix $A$ and has the advantage that the hydrologic responses drive the result more directly, but the allocation of conductors may be inefficient.

For either approach, the resolved pattem is not guaranteed to be the same as the one in field, and for this reason we call it an equivalent system. The equivalent systems are non-unique. This means we can find a range of systems which behave the same way the real system behaves. The range of systems can then be used to make a range of predictions.

Once an equivalent pattem has been determined, we can compare the behavior of the model to that of the observations. In some cases, it may be that pattem alone does not allow one to create a sufficiently accurate model. One could refine the template in the 
vicinity of the poor match in order to provide for greater contrasts in conductance. However, for very large contrasts one might wish to use annealing to discem the variability of conductance among elements. This has been observed in one case (Davey et al, 1990).

The process of annealing is summarized as follows. In situ well tests are simulated on the model using TRINET. We then define the "energy," $Q_{C}$ of our current model configuration, (referred to as $C$ ), as the squared sum of the differences between the measured and simulated heads, taken at a set of locations at discrete time intervals. Next we change the model by switching a randomly chosen channel "on" (i.e. conducting) if is it is "off' (i.e. non conducting) or vice versa and then repeat the well test simulation. If the changed model gives simulated well test data closer to the real measured values, i.e. $Q_{C}$ is decreased, then the changed model is kept. However, if $Q_{C}$ is increased by the change, then the change will be kept with a certain probability, $P$, which is a function of a weighting factor, T, (called the "temperature"), and $Q_{C}$. The temperaure is decreased as the number of iterations increases to make it more and more unlikely that an unfavorable change will be accepted. At first, a high value of $T$ allows the algorithm to jump up out of local minima and continue searching for a better region of the function. Later, lowering the temperature tends to confine the search for a minima, so the algorithm can converge.

\subsection{Annealing Theory}

This optimization technique employs an analogy between optimizing a function with many local minima and the process of annealing a metal to reach its lowest energy state. Thus, simulated annealing can be viewed as a process of minimizing an energy function over a set of possible configurations of elements.

Simulated annealing has been defined in analogy with the thermodynamic process of cooling or annealing of a metal (Kilpatrick, et al., 1983; Tarantola, 1987). A system composed of atoms of a metal in thermal equilibrium at a temperature $K$, has a range of possible energies, depending on the configuration. Thermodynamically, low energy states are more likely, but at any temperature, there is still some chance of being in a high energy state. Metropolis et al. (1953) used this conception of energy states to simulate changes in a thermodynamic system.

To use simulated annealing on a general problem, one needs a set of possible configurations, a way of randomly changing the configurations, a function one would like to minimize, and an annealing schedule of temperature changes (Press, et al., 1986).

We can define the set of all possible configurations using our base model: $M$, i.e. the template which is the set of all possible pipes or channels. The channels have two possible states: they are either off or on. The set of all possible configurations is the sei of all combinations of off and on pipes. Let $C=\left\{C_{\mathrm{m}}, \mathrm{m}=1 \ldots M\right)$ denote a configuration of on and off pipes, where $C_{\mathrm{m}}$ is a binary random variable associated with each pipe.

We now change the system. First, we use some probability function to randomly select a pipe. If the pipe is on, we tum it off, and if the pipe is off, we tum it on. In this way we find a new configuration which is in the the neighbortood of $C$ where the neighborhood of $C$ is all configurations one step away from $C$. Let $C$ be the configuration at iteration $n$ and $G_{C}$ be its neighborhood. When we anneal the system, we randomly select a configuration $C^{\prime}$ from $G_{C}$ at each iteration $n$, and compare the two energy functions. 
The energy functions we use are a measure of the difference between the observed and the simulated system response. We consider energy functions of the form:

$$
Q=\sum_{j}\left|f\left(o_{j}\right)-f\left(s_{j}\right)\right|^{m}
$$

where

$$
\begin{aligned}
\mathbf{o}_{\mathrm{j}} & =\mathbf{a} \text { vector of observed responses } \\
\mathbf{s}_{\mathbf{j}} & =\mathbf{a} \text { vector of simulated responses } \\
\mathbf{m} & =\mathbf{a} \text { constant } \text { and }
\end{aligned}
$$

$f(\cdot)$ is a real monotone function.

The observed measurements could be hydrologic, geological, or geophysical.

The energy function, scaled by the temperature, is used to decide whether the system should make a transition to a new configuration. The temperature is lowered as the algorithm progresses, to make it increasingly unlikely that a transition to a higher energy state will occur.

We assume a probability distribution on the configurations can be expressed as a Gibb's Distribution:

$$
\mathrm{P}(C)=\frac{1}{k} \mathrm{e}^{-\left[\frac{\mathrm{Q}(C)}{\mathrm{T}}\right]} .
$$

Where $k$ is a nommalizing constant which assures that the sum of the probabilities of all possible configurations is unity. We assume that this constant exists, but it is very difficult to evaluate because we must know the energy for every possible configuration to compute $k$. So we can not compute the absolute probability of any given configuration because we do not know $k$. However, we can compute the relative probability of any given configurations. For instance, we could say that a configuration would be twice as probable as another.

Further, we know that if the probability function is a Gibb's distribution then this is equivalent to modeling $C$, the current configuration as a Markov Random Field. A Markov Random Field exists on a neighborhood system, $G$ if the probability defined meets two conditions. The first condition is that the probability of selecting any configuration in the system is greater than zero. The second is that the probability of making a transition from $C$ to $C^{\prime}$ depends on $C, C^{\prime}$ and whether $C^{\prime}$ is in the neighborhood of $C$. Past history, such as the configuration we selected before $C$, does not tell us anything about the probability of moving from $C$ to $C^{\prime}$. So, the probability of moving from one configuration to another can change with the iteration, but does not depend on which configurations have been examined in the past. This means we can examine a series of configurations without remembering how we moved from one to the next and we can still compute the relative probability of each configuration.

At each iteration $\mathrm{k}$, given $C, \mathrm{G}_{C}$, the neighborhood, and $\mathrm{T}$, the temperature, we can find a matrix of transition probabilities. The probability we will move from configuration $C$ to $C^{\prime}$, given our current configuration $C$ is equal to the probability that we select $C^{\circ}$ to compare with $C$, multiplied by the probability that the system would make the transition to a given $C^{\prime}$. That is: 


$$
\mathrm{P}\left\{C \rightarrow C^{\prime}|C|=\mid \begin{array}{lr}
0 & \text { if } C^{\prime} \notin \mathrm{G}_{C} \\
\mathrm{P}\left(C^{\prime} \mid C\right) \cdot 1 & \begin{array}{rl}
\text { if } C^{\prime} \in \mathrm{G}_{C}, \mathrm{C}^{\prime} \neq \mathrm{C} \\
\mathrm{Q}\left(C^{\prime}\right)-\mathrm{Q}(C) \leq 0
\end{array} \\
\mathrm{P}\left(C^{\prime} \mid \mathrm{C}\right) \cdot e^{-\left[\frac{Q(C)-Q(\mathrm{C})}{\mathrm{T}}\right]} & \begin{array}{r}
\text { if } C^{\prime} \in G_{C} C^{\prime} \neq \mathrm{C} \\
G(C)-Q(C)>0
\end{array}
\end{array}\right.
$$

and the probability of not accepting the change to $C^{\prime}$ is:

$$
\begin{gathered}
\mathrm{P}\left(C \rightarrow C^{\prime} \mid C\right)=\mathrm{P}(C \rightarrow C) \\
=1-\sum_{\left\{C^{\prime}: Q\left(C^{\prime}\right) \leq Q(C)\right]} \mathrm{P}\left(C^{\prime} \mid C\right)-\sum_{\left[C^{\prime}: Q\left(C^{\prime}\right)>Q(C)\right]} \mathrm{P}\left(C^{\prime} \mid C\right) \cdot e^{-\left[\frac{Q(C)-Q(C)}{\mathrm{T}}\right]}
\end{gathered}
$$

It remains to discuss the temperature schedule. The schedule is used to lower the temperature as annealing progresses. Physically, this means that as annealing progresses we are less and less likely to keep changes which increase the energy of the system. At this time, there is a theory which relates the temperature schedule to the convergence properties of annealing. This theory (Hajek, 1988) shows that a temperature schedule which is inversely proportional to the log of the iteration number will converge in probability to a set of minimum energv states.

However, a temperature schedule of this type is prohibitively slow. Further, we do not necessarily want to find the minimum energy configuration. We are only interested in finding several good solutions, not in certain convergence in probability to a set of minimum energy states. Therefore, use of Hajek's temperature schedule is over constraining for our purpose.

The temperature schedule we use here is only justified heuristically: it works. We have followed the suggestion of Press, et al. (1979) and decreased the temperature after a number of iterations which is sufficient to result in accepting $\mathbf{5 0}$ changes. Each interval of the schedule with constant temperature is called a step. At the end of each iteration, $k$, the temperature, $T_{k}$, is decreased using a geometric series,

$$
\mathrm{T}_{k+1}=\mathrm{T}_{\mathbf{k}} \mathbf{t}^{k}
$$

where

$$
0<\mathrm{t}<1 \text {. }
$$

The initial temperature is chosen such that it is of the same order of magnitude as the energy difference between the first two configuration. This is done in an attempt to scale the energy difference between successive configurations to something between zero and one. Other choices of temperature schedule are possible and these are currently the topic of research.

\subsection{Bayesian Analysis}

In the case where we have a series of similar in situ tests available, but the prediction we wish to make is for a dissimilar test, i.e. one that is far away from all the other tests, 
or one that has distinctly different flow conditions, we use the Bayesian approach or method of regularization to incorporate prior physical information into the model. This approach is based on the fact that quantitative information such as the range of possible responses might be available even if there is no actual measurement. Using this information, we can constrain the model to give a response within this range. In this case, we might have some general information about the area, such as a range a certain response should fall in. This information can be used in the annealing process, by adding a step which makes it more likely that configurations which have a calculated response value in this range will be accepted. The values predicted by the final models may be used to update your estimate for this response.

For example, suppose we want to predict the flow to a borehole in the model at a place far from the present boreholes. We have as prior information that steady state drawdown values have been observed to be between 10 and 20 . That is

$$
h \in[10,20]
$$

We then anneal using the energy function based on the known borehole responses. However, every time we change the configuration we do the following:

1. Add the new borehole to the configuration and calculate the drawdown, $h$ at that borehole.

2. Compute a new energy function $Q^{\circ}$ as follows:

$$
\begin{array}{cl}
0 & \text { if steady state value }=h \in[10,20] \\
Q^{\prime}(C)=[h-10]^{2} & \text { if } h<10 \\
{[h-20]^{2}} & \text { if } h>20
\end{array}
$$

3. Add $Q^{\prime}$ to the energy calculated for the known responses, $Q$

4. If $\mathrm{Q}^{\prime}\left(C^{\prime}\right)+\mathrm{Q}^{\prime}\left(C^{\prime}\right)$ is an improvement over $\mathrm{Q}(C)+\mathrm{Q}^{\prime}(C)$, keep $C^{\circ}$ If not, use usual annealing probability to keep or reject $C^{\prime}$

5. Remove new borehole and continue

\subsection{The Annealing Computer Algorithm}

The finite element code, TRINET, simulates steady or transient flow in a fracture network. The code is used to solve for the head distribution as a function of time in the fracture mesh created by the mesh generator in response to a simulated well test. For example, the head measurements from transient constant flux well tests could be simulated with TRINET. Now, the head versus time curves from the simulated well tests are examined and the values of transmissivity and storativity assigned to the elements are scaled in order to achieve as good a match as possible to the observed draw down curves. This corresponds to shifting the well test data to match the Theis type curve (Freeze and Cherry, 1979). We then define the "energy," Q (C) of our current model configuration as the squared sum of the differences between the observed and calclilated head values at different locations (j) and specified times (i):

$$
Q(C)=\sum_{m} \sum_{i}\left[H_{o b s}(i, j)-H_{\text {sim }}(i, j, C)\right]^{2}
$$

In the case presented here, we do not have a transient test, only a single steady state example. So the energy function is just computed at the points where head is 
monitored:

$$
Q(C)=\sum_{j}\left[H_{o b s}(j)-H_{\mathrm{sim}}(j, C)\right]^{2}
$$

The computer algorithm can be written in 4 steps.

1. Randomly select a beginning configuration from the set $\Omega$. Calculate $Q(C)$.

2. Randomly select a pipe from $\mathbf{M}$. If the pipe is in $C$, delete it, if the pipe is not in $C$, add it. This is the new configuration, $C^{\prime \prime}$.

3. Calculate $\mathrm{Q}(C)$.

If $\mathrm{Q}(C)>\mathrm{Q}(C), C \rightarrow C^{\circ}$.

If $\mathrm{Q}(C)<\mathrm{Q}(C)$, use a random number and the transition probability to decide if $C \rightarrow C^{\circ}$ or $C \rightarrow C$. If $\mathrm{Q}(C)$ is accepted then $\mathrm{Q}(C)$ becomes $\mathrm{Q}(C)$ for the next iteration.

4. If the end of step $k$ of the annealing schedule has not been reached, return to 2 and continue. If the last iteration of step $k$ was reached, reduce $T_{k}$ to $T_{k+l}$, return to 2 and continue. If the last step $k$ of the annealing schedule was reached, stop if no change was made in the configuration after some large number of iterations.

\subsection{A Synthetic Example Case}

An example of annealing results is shown in Figure 4.1. Here we have created a synthetic fracture system (Figure 4.1a) and used it to create synthetic well test data. Then, we create a regular grid to use as a template (Figure 4.1b) and apply annealing to find a configuration which matches the synthetic well test data (Figure 4.1c). This example shows that the annealed result roughly reproduces the connection between the wells. Major gaps similar to those in the "real" system have analagous gaps in the annealed system.

A full suite of synthetic cases is being developed to study the effect of template geometry, temperature schedule, weighting functions etc (Davey et al, 1990). This suite should give us experience that can be applied to cases where the true geometry is really unknown. 

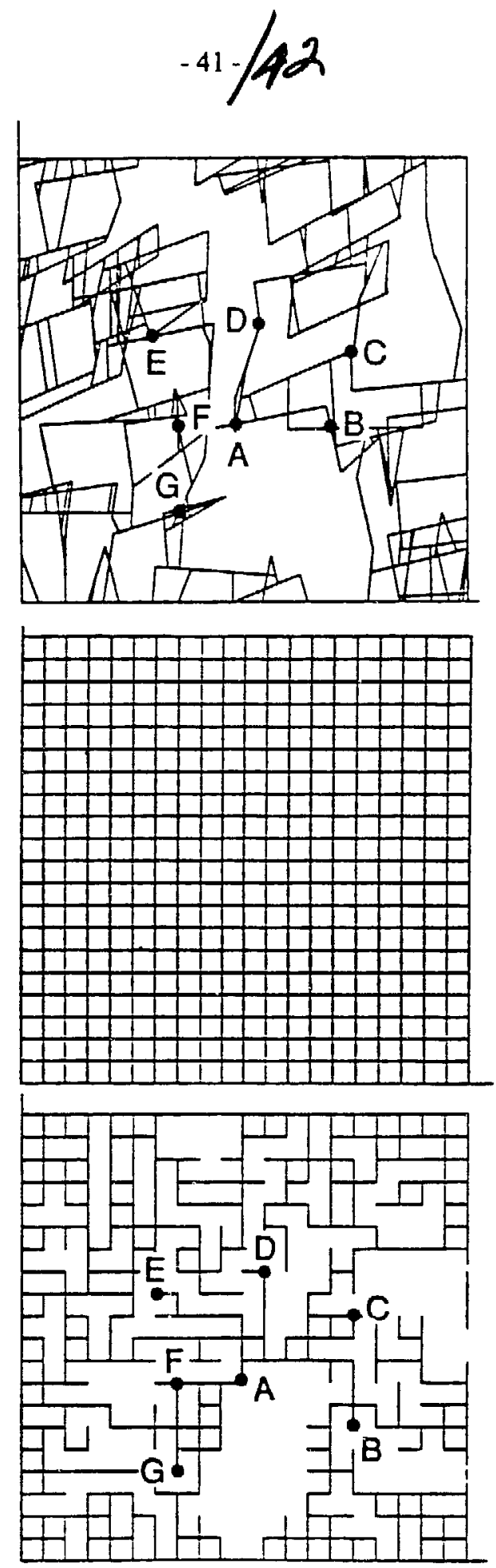

XBL $891-6142$

Figure 4.1. (a) A "real" fracture network where we have generated "real" data on well test in hole A and monitoring in holes B through G; (b) Template model; (c) The pattem of conductors resulting from annealing. 


\subsection{MEASURES OF UNCERTAINTY}

After annealing has been used to identify model configurations of conductors which match the observed insitu behavior, the models can be used to make predictions of other flow situations. In this example, we first apply boundary conditions to simulate the well tests used for annealing. When annealing is completed, we shut in these wells in the model, and create new boundary conditions appropriate for predicting some new flow conditions. We now wish to determine how good these predictions are. There are two approaches to this problem: Prediction error (or cross validation), and sensitivity and relative likelihood. These are discussed below.

\subsection{Prediction Error}

The first approach, and by far the best, is to use the annealed model to make a series of predictions similar to the one which is required, but for which the quantities predicted have actually been measured. In other words, we ignore some of the data and use the model to predict the data we ignore. Then, for each prediction we can calculate a prediction error and the root mean square prediction error is a direct measure of how well we can make similar predictions. This is the best way because all the uncertainties in the model itself are integrated into an error which can actually be measured.

For example, suppose we have 5 wells, and a cross-hole well test from another, sixth well to all 5 wells. This gives an observed response $\mathrm{H}_{\text {obs }}^{\mathrm{i}}$, at cach well, $\mathrm{i}$. We would like to know the prediction error associated with ssing our annealing model to predict the response at a seventh nearby well. For example, if we were using transient well test data in the general case we could do the following:

1. Leave the well test curve for well $i$ out of the energy function

2. Anneal $n$ times using the other 4 well test curves until the energy is sufficienty low.

3. For each end configuration, $C_{l}^{-\mathrm{i}} \cdots C_{\mathrm{n}}^{-\mathrm{i}}$, calculate a predicted well test curve for well $\mathrm{i}$. These head versus time curves are $\mathrm{H}_{1}^{-1} \cdots \mathrm{H}_{\mathrm{n}}^{-1}$

4. Calculate the prediction error for well $\mathrm{i}$ :

$$
P E^{2}(i)=\frac{\sum_{j=1}^{n}\left(H_{j}^{-i}-H_{o b s}^{i}\right)^{2}}{n}
$$

The total prediction error is:

$$
P E=\left(\frac{\sum_{i=1}^{5} P E(i)}{5}\right)^{1 / 2}
$$

PE is then an estimate of the error involved in using the annealing model to predict the response of any other well in the vicinity. The more and different kinds of responses we can use to calculate the prediction error, the more confidence we will have in the predictions that are made. If there are enough cases to allow us to calculate an empirical distribution of prediction errors, then we can put confidence limits on the prediction. 


\subsection{Sensitivity and Relative Likelihood}

Unfortunately, a good estimate of prediction error will require a good sample of in situ tests. As discussed above under section 1.3 on validation, this is not always available. When prediction error can not be estimated, we can separate the uncertainty into two components: uncertainty in the conceptual model and uncertainty in the parameters. The uncertainty in the conceptual model can be approached by trying different conceptual models. For example, we can compare the zone model to the grid model. However, there are a very large number of possible conceptual models and we must invoke a "peer review" to decide if we have looked at enough of these.

This leaves uncertainty in the parameters of the model. To approach this problem, we can perform studies which vary the parameters to see what effect they have on the prediction. In our case, we have essentially three kinds of parameters: boundary conditions, the value of the conductance assigned to each conductor, and finally the arrangement of conductors in the model. A study of the sensitivity to boundary conditions can be done by trying different scenarios such as a free surface boundary or adding the drifis to the model. As for the conductances, as long as we use models with constant conductance, any prediction of steady flow rate will be directly proportional to the conductance assigned to the elements, so sensitivity to this parameter is known a priori.

Finally, we can examine the uncertainty in the arrangement of the conductors. looking at a series of different arrangements, and for each one we can calculate the energy function. The lower the energy, the more likely is the arrangement. So, although we can not say what the probability of each arrangement is, we can determine the relative likelihood of any predictions that we make. If the energy function we use in the annealing process is a combination of energy functions for different types of observations, we may rank the final solutions differently for each type of response.

There are several ways which we could choose different configurations of conductors to compare. The simplest is to use a series of configurations defined at the end of an annealing process. These configurations are easily available, but they will probably be very similar to each other. Another way to find different configurations is to perform annealing several times, each time staning with a different initial configuration. For example, we could start with different initial arrangements all with the same percentage of elements present, or we could start with different percentages. For a complete treatment, these exercises should be performed. They are not included here because the available data is not complete enough to warrant this effort.

As these different configurations are developed, one would also want to examine them to see if they are alike geometrically. If models started from different initial cases all develop the same characteristics, we would want to examine these characteristics in light of the geologic and geophysical evidence. For example, the models may all show lack of connection between two points, or they may all show a preference for strike oriented channels versus dip oriented charinels.

Mathematically, the relative likelihood is calculated as follows. Assume we have an annealed model with end configurations:

$$
C_{\mathrm{i}} \mathrm{i}=1 \cdots \mathrm{n}
$$

For each configuration, we know the the energies

$$
Q\left(C_{\mathrm{i}}\right) \mathrm{i}=1 \cdots \mathrm{n}
$$


and the final temperature $T$. So we can compute the relative likelihoods of the $i$ configurations:

$$
\mathrm{e}^{\frac{-Q\left(C_{1}\right)}{\mathrm{T}}} \cdots \mathrm{e}^{\frac{-Q\left(C_{0}\right)}{\mathrm{T}}}
$$

and rank the estimates from most likely to least likely using the relative likelihoods. 


\subsection{PREDICTION OF INFLOW TO THE D-HOLES}

\subsection{Development of a Synthetic Cross-hole Test}

At this stage in the hydraulic investigation of the SCV site, there are no formal, well controlled cross-hole well tests available. In order to gain experience with the annealing technique and produce a preliminary estimate of the flow into the D-holes, a synthetic cross-hole test was produced based on a variety of data available for the SCV site. This data consisted of ad hoc cross-hole tests performed by British Geologic Survey (BGS) and the record of heads in the boreholes.

David Holmes of BGS conducted three ad-hoc cross-hole tests by opening W2 and monitoring sections in N3, N4, and W1. Figure 6.1 (D. Holmes in Olsson et al., 1989 shows how the boreholes responded. Some zones did not respond and some responded very quickly. The test durations were too short to achieve steady-state conditions. At the same time, the transient data, shown in Figures 6.2a, b, and c (D. Holmes, personal communication), was at best qualitative because the tests were conducted while the system was in a recovery period due to other perturbations. Also, the test was started without waiting for the packer inflation effect to diminish or for the zone pressure to build up to the environmental head. Therefore, the data is a complex combination of all three effects. We did not attempt to analyze these effects and did not use the transient data directly in the quantitative annealing procedure. However, qualitatively these tests offer valuable information of the major hydrologic features. In comparison to the single hole tests, the scale of these cross-hole tests is much more representative of the overall size of the SCV site and the large hydrologic features.

Based on the transient results plus the record of hydraulic heads in the holes, a synthetic steady state test was fabricated. The steady flowrate from W2 was extrapolated to estimate the steady-state flow rate (Figure 6.3 ) of 10 liters/min. The corresponding estimation of steady state head in N3, N4, and W1 was found by extrapolating the head values in those holes during the period when W2 was opened for prolonged period of time (Figure 6.4). Wherever possible, these heads were assigned to specific zones in the boreholes based on the responses observed during the ad hoc test. In other words, during the ad hoc tests, the observation holes were divided into several sections. Some sections did not respond to opening $W 2$, whereas others responded very rapidly. In these cases, the responding zone was assigned the observed head.

The annealing case is summarized in Figure 6.5 and in Table 6.0b. Figure 6.5 shows the $\mathrm{N}$ - and W-boreholes, plus the "fins" that are used to connect the boreholes to the hydrologic zones. Each fin represents the intersection of a zone with the borehole. The black fins are those where we have determined a value of head to use in the calculation of annealing energy based on the ad hoc cross hole tests plus the head record. The gray fins in W2 are held at zero head to simulate the opening of W2. The white fins are those for which we have no record of response to opening W2. In these cases, we could not be sure that there was no response under steady conditions. We only knew that no response was observed under the limited testing period. Therefore, the value of head at these fins is calculated but not used in the annealing process. When a zone does not respond, the annealing algorithm will disconnect the zone, so uncertainty about lack of response is a significant loss of information. 


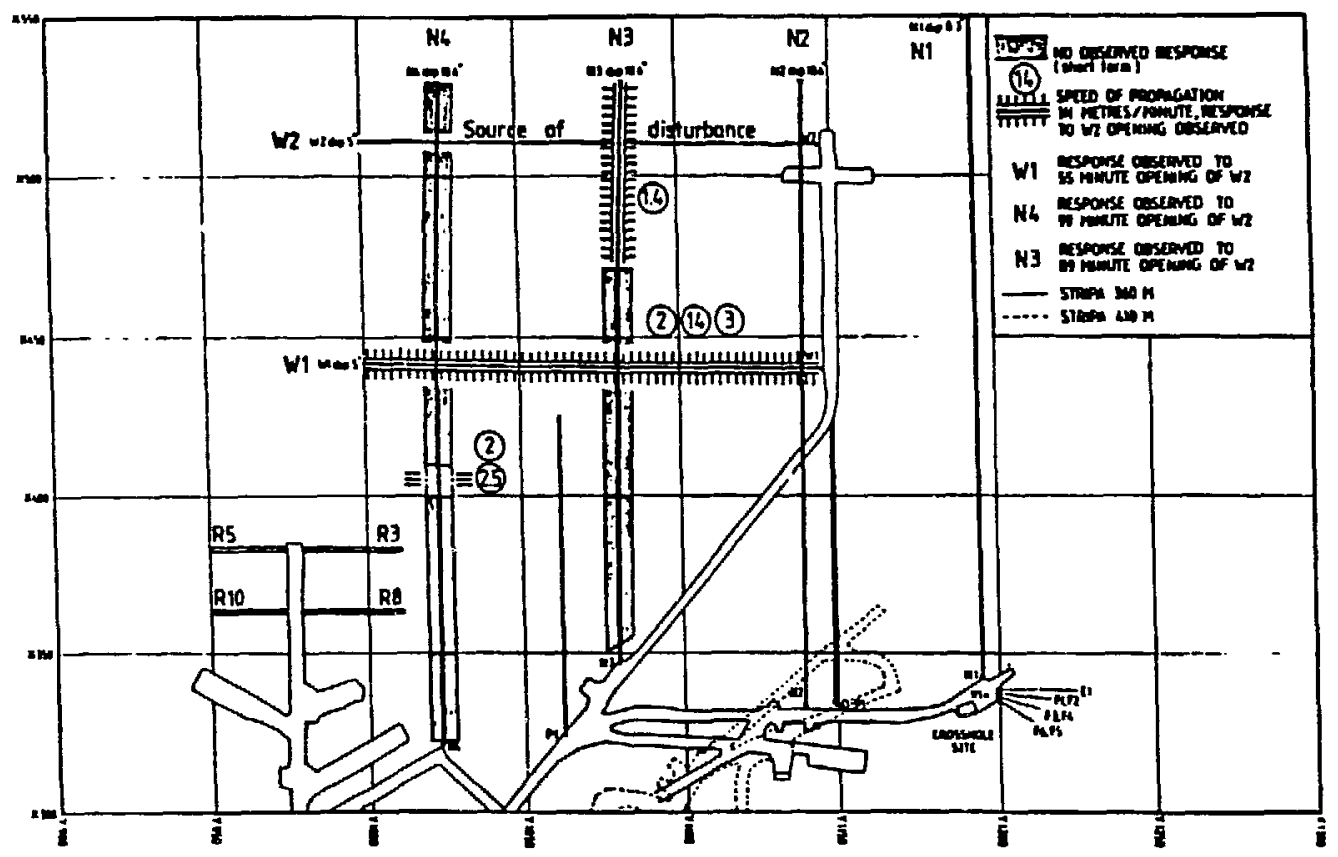

Figure 6.1. Responses in the SCV block caused by opening W2. Three different tests performed, sequentially monitoring the responses in N4, N3 and W1. In the case of $\mathrm{N} 4$ and $\mathrm{N} 3$, packers were placed in the holes so that different responses could be seen in different parts of the well. In the case of W1, the hole was undivided (after Holmes, in Olsson, 1988). 
(a)

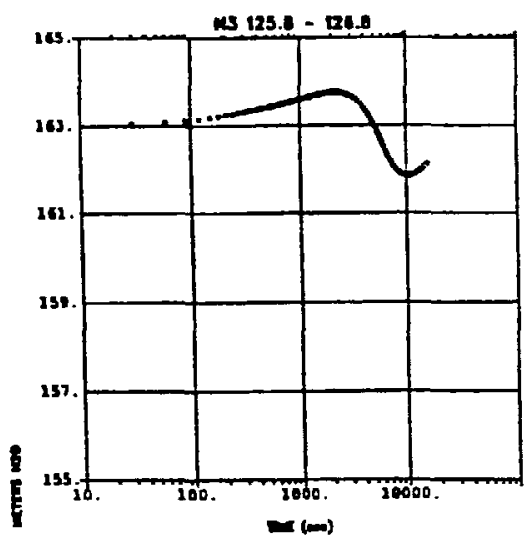

(b)

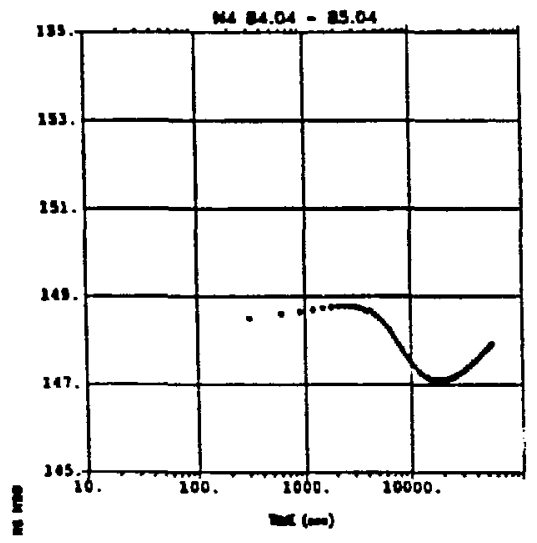

(c)

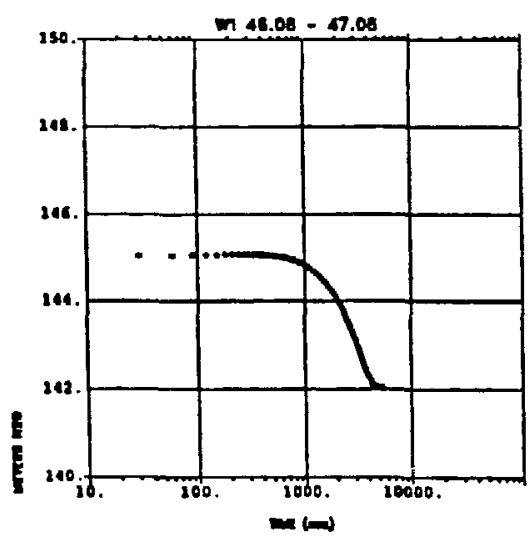

Figure 6.2. (a) Response in N3 to opening W2. (b) Response in N4 to opening W2. (c) Response in W1 to opening W2. 


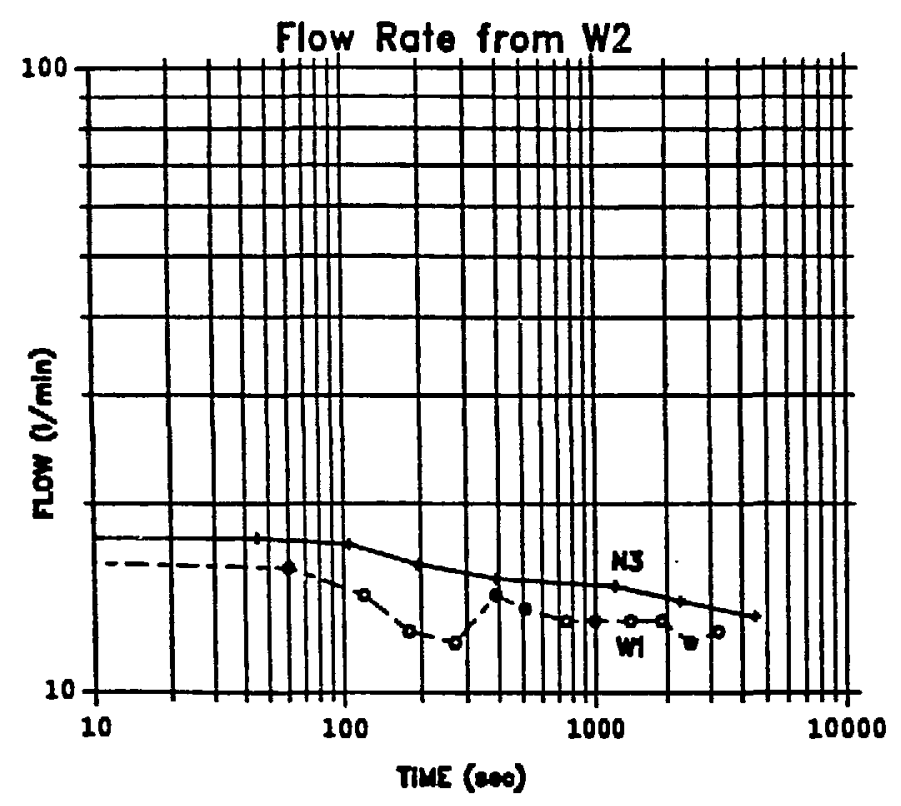

Figure 6.3. Flowrate in W2 recorded during two periods: when N3 was monitored and when W1 was monitored.

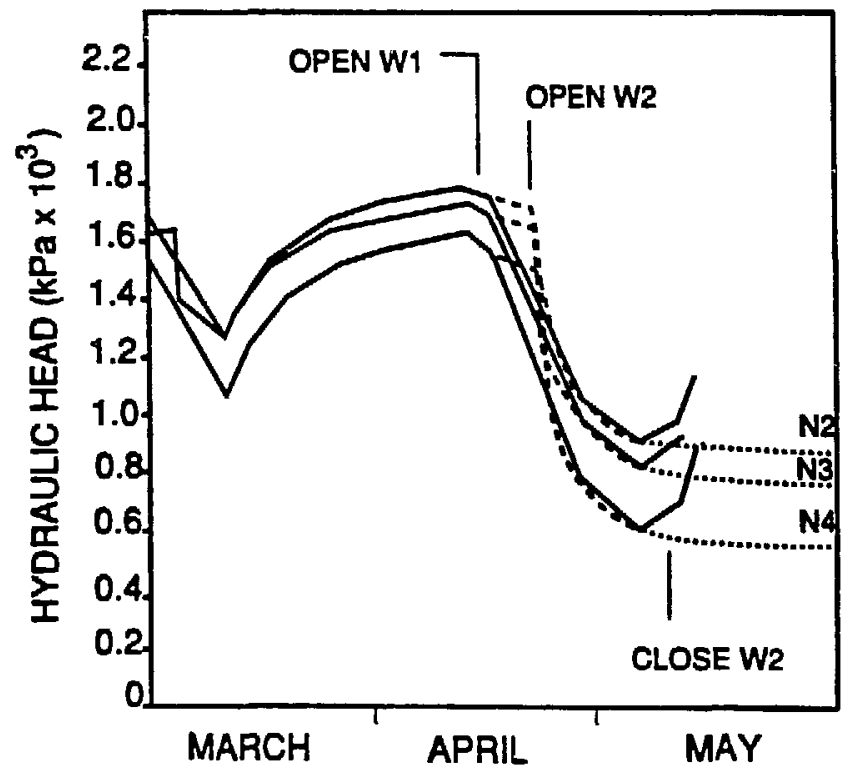

Figure 6.4. Responses in N2, N3, and N4 (entire hole) to opening W2 (from the head records, 1987). 


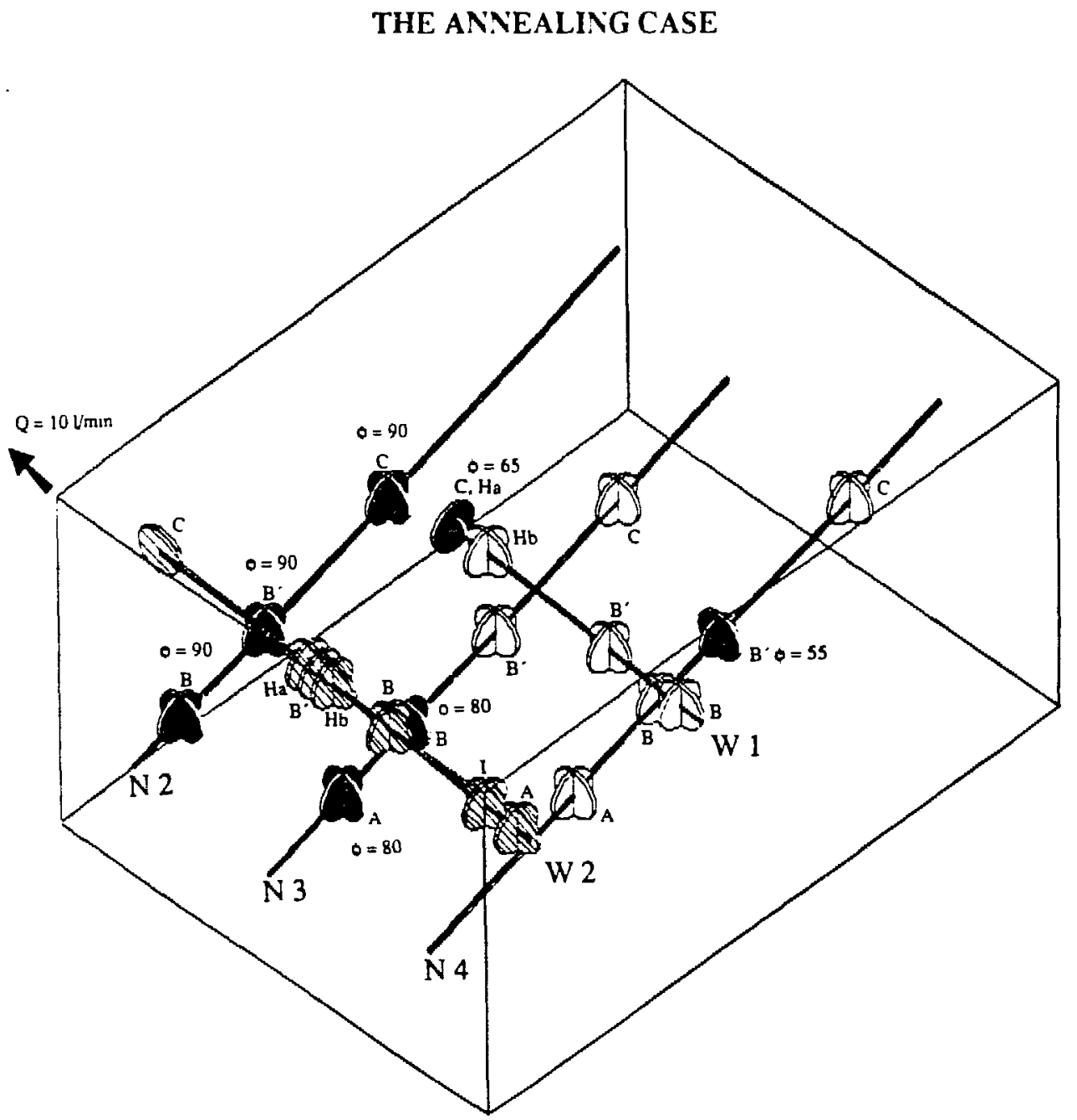

"Observed" heads (m) based on synthetic steady flow from W2, used in calculating the energy for annealing.

Value of head not "observed", predicted by annealing.

Open hole, $\phi=0$

Figure 6.5. Summary of the synthetic annealing case. 
Table 6.1. Annealed Borehole Sections and Head Values

\begin{tabular}{|c|c|c|c|c|}
\hline Hole & Depth (m) & Zones & Head $(m)$ & Flow $(1 / \mathrm{min})$ \\
\hline N2 & $0-207$ & $\mathrm{~B}, \mathrm{~B}^{\circ}, \mathrm{C}$ & 90 & 0 \\
\hline N3 & $131-189$ & $\mathrm{~A}, \mathrm{~B}$ & 80 & 0 \\
\hline N4 & $87-100$ & $\mathrm{~B}^{-}$ & 55 & 0 \\
\hline Wl & $43-51$ & $\mathrm{H}_{\mathrm{a}}, \mathrm{C}$ & 65 & 0 \\
\hline W2 & $0-147$ & $A, B, B^{\prime}, C, H_{a}, H_{b}, I$ & 0 & 10 \\
\hline
\end{tabular}

\subsection{Boundary Conditions and the Starting Configuration}

There are two possible choices for setting the boundary conditions. A regional mine scale hydrologic model has been constructed (ref. J. Gale in Olsson, 1989). This model could be used to pick out the hydraulic heads at the boundaries of the SCV block and assign these to the zone model. This option was rejected because changes in the properties of the zones model would have a large effect on the boundary heads. Thus, the mine model would have to be directly coupled to the zone model in order to predict the heads on the boundary. This option is not practical in conjunction with annealing.

An altemative is to base the choice of boundary conditions on head observations in the boreholes. The SCV block is situated in a large zone of depressed heads which represent the steady drainage created by the mine. Therefore, whatever perturbation there are in the SCV block must be superimposed on this existing condition. Shut-in heads throughout and around the SCV block are surprisingly consistent and centered about $200 \mathrm{~m}$ (Figure 6.6). Therefore we choose the altemative of making the boundary conditions constant head equal to $200 \mathrm{~m}$. Although we do not expect the boundary conditions to be uniform around the edges of the zones we have too little data to have any more resolution than setting the boundary conditions to one estimated figure. However, these boundary conditions do represent a degree of approximation consistent with the rest of the assumptions we have made so far.

Using $200 \mathrm{~m}$ head boundary conditions applied directly on the edges of the block and opening W2 to atmospheric pressure results in the model calculating heads in the other $\mathrm{N}$ - and $\mathrm{W}$-holes which are too high compared to the actual heads that were observed when W2 was opened (Figure 6.7). Further, we know that pressure perturbations in the SCV block are felt much farther away than the boundary of the SCV block. We conclude that having fixed head boundaries coinciding with the boundary of the SCV block is unrealistic because these boundaries are hydraulically too close to the $\mathrm{N}$ - and W-holes to allow for the observed head drops.

In order to get a better representation of the hydraulic conditions in the block, we connected each zone to boundaries set at $200 \mathrm{~m}$ head far away from the block. The connection was made through channels shown in Figure $6.8 \mathrm{a}-\mathrm{g}$ which had conductance equal to that of the channels in the block. The distance of $1000 \mathrm{~m}$ was chosen so that the simulated heads in $\mathrm{N}$ - and $\mathrm{W}$-holes are, on average, close to the observed heads. This distance is larger than the distance to the water table, but using $1000 \mathrm{~m}$ is numerically equivalent to setting the permeability of the rock surrounding the SCV block to be much lower than that of the zones. So, we are effectively controlling the head drop to the boundary of the block, but we are not concemed with what actually causes the head drop outside of the block. 


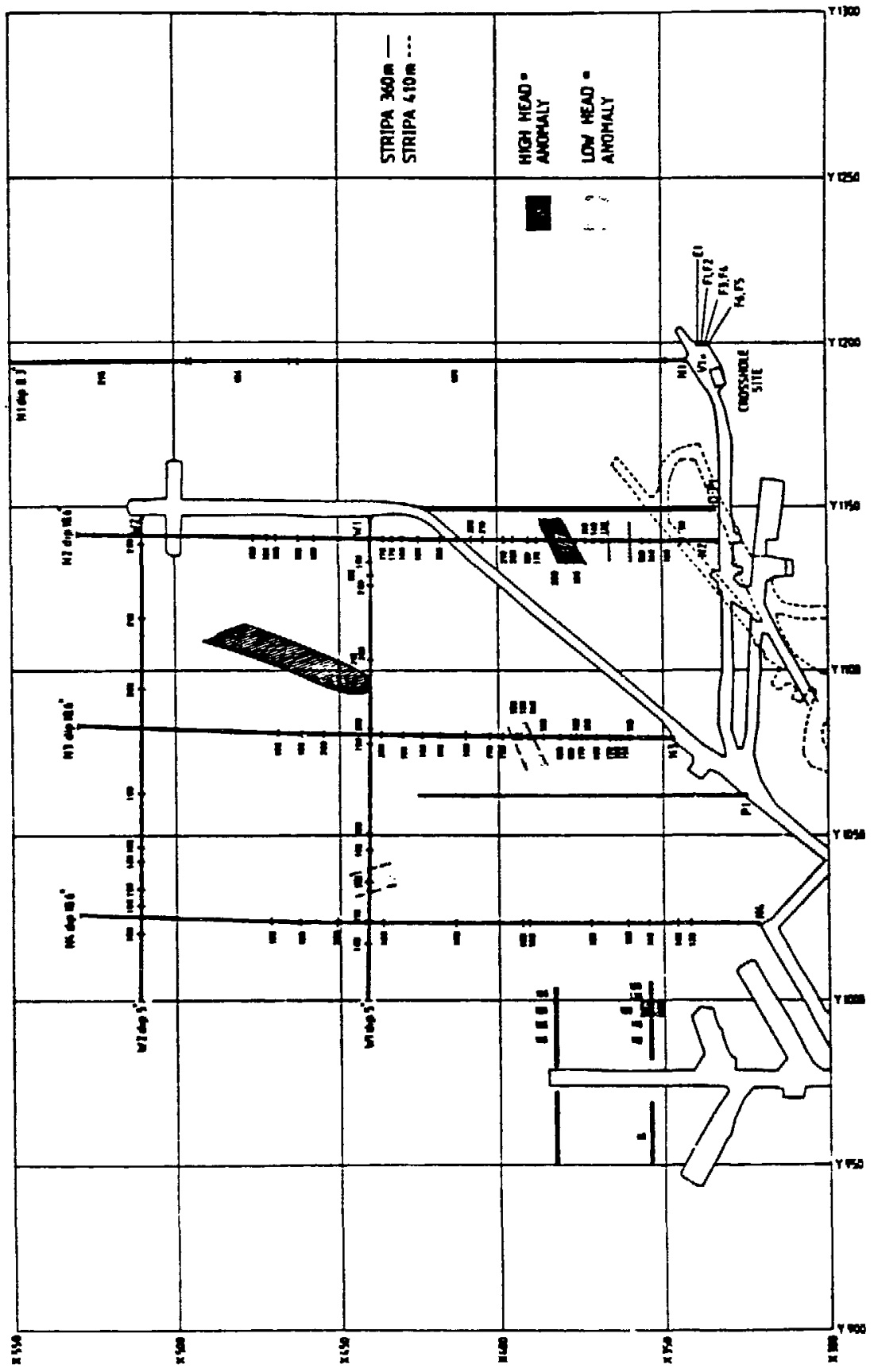

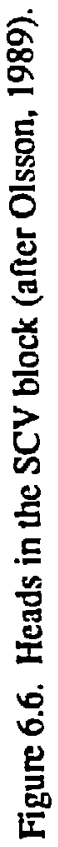




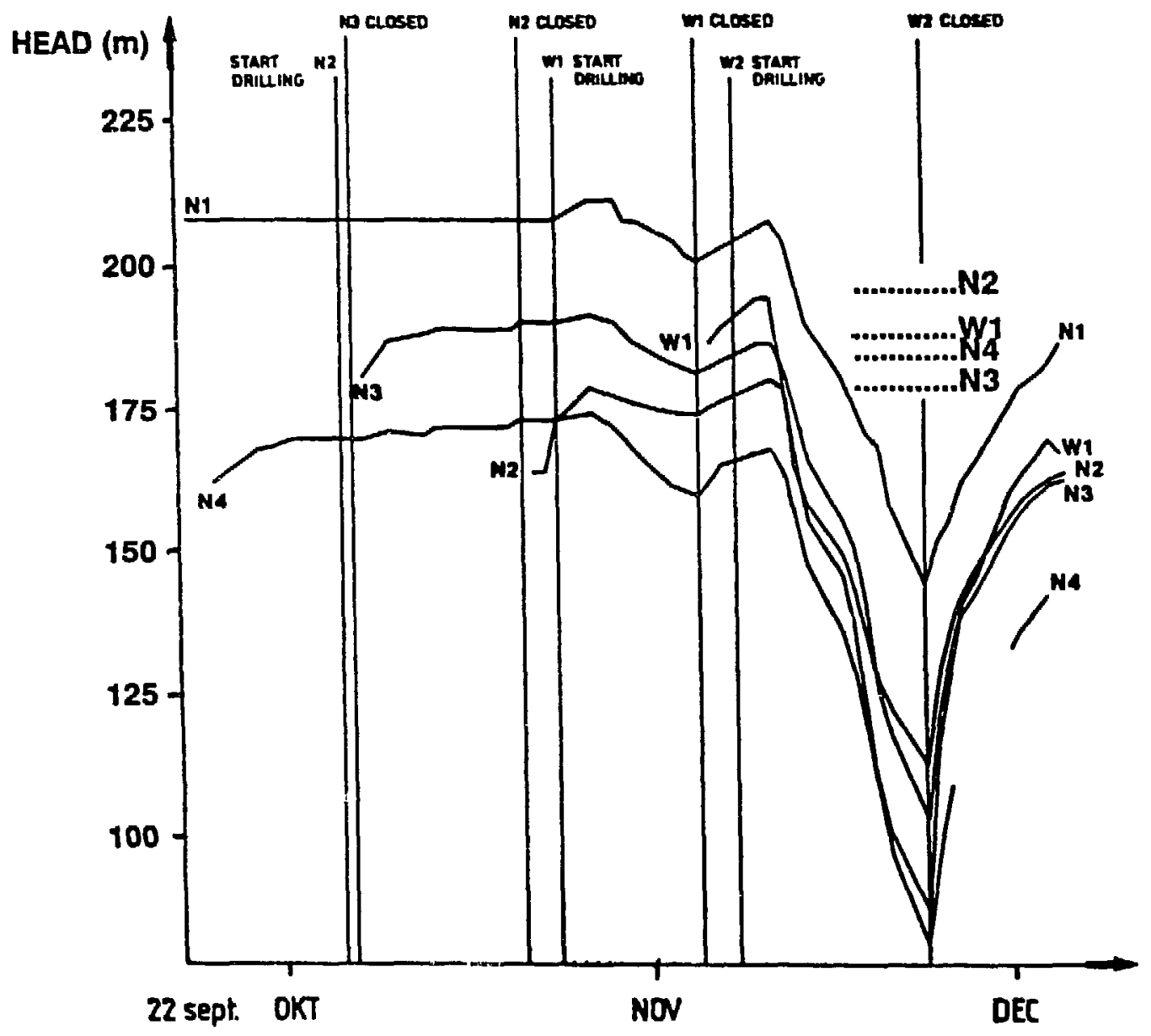

Figure 6.7. Heads that result in the $\mathrm{N}$ - and $\mathrm{W}$-holes when $\mathrm{W} 2$ is opened and head $=200 \mathrm{~m}$ boundary conditions are applied directly to the SCV block boundaries. 


\section{Extended Boundaries, Zone A}

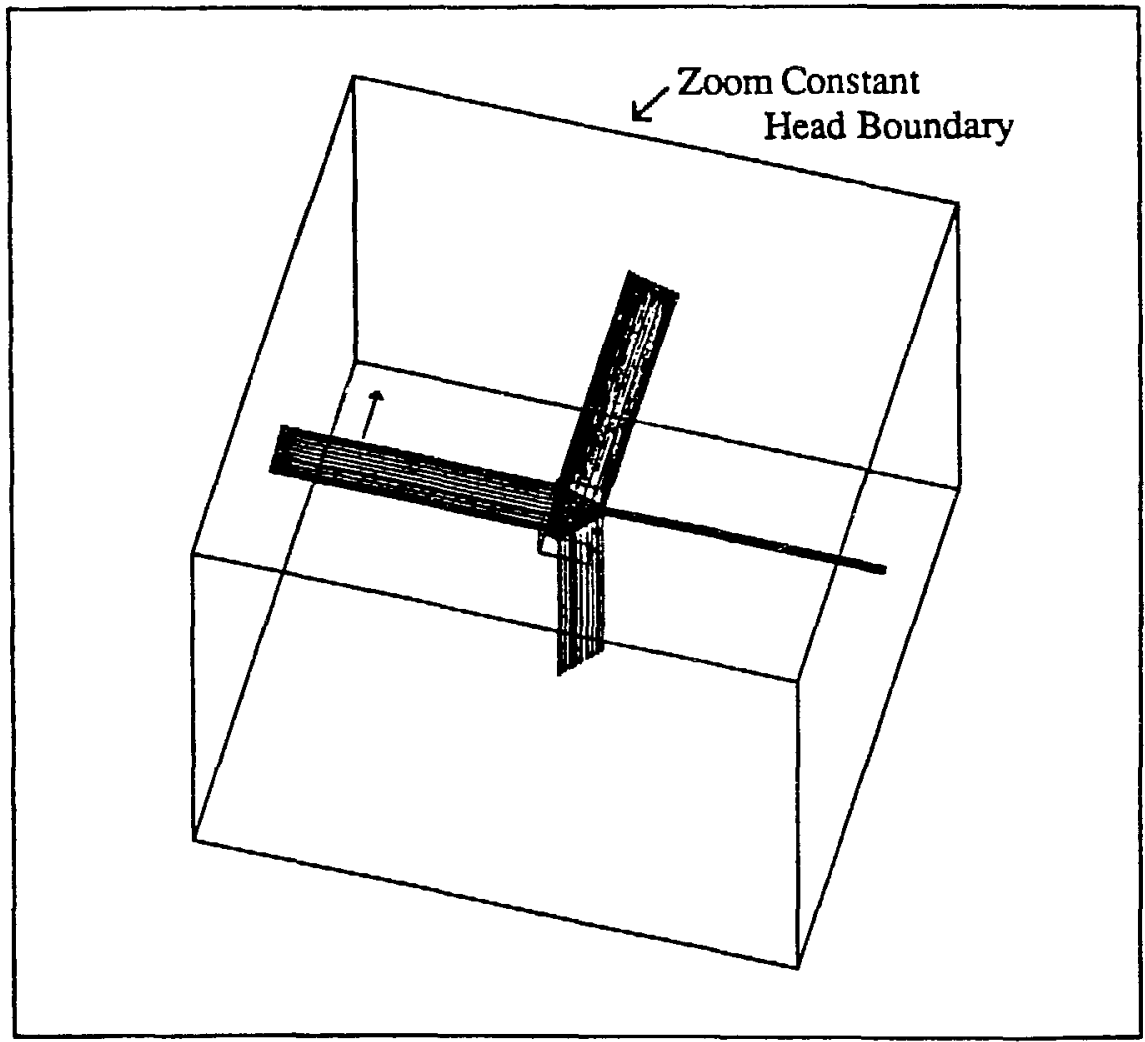

Figure 6.8a. Perspective plot showing how the zone $A$ is connected to the boundaries of the model which are held at $200 \mathrm{~m}$ head. 
Extended Boundaries, Zone B

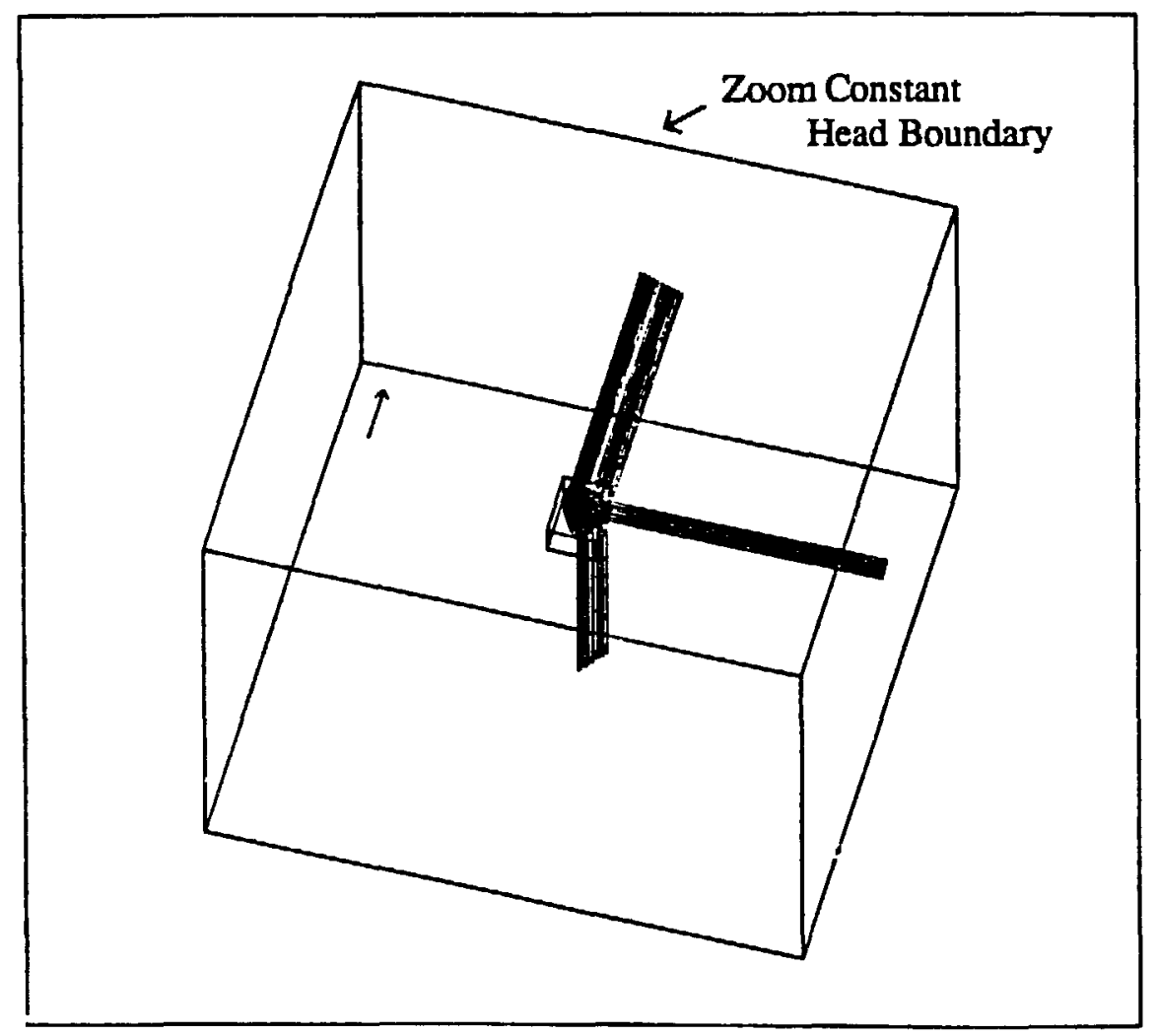

Figure $6.8 \mathrm{~b}$. Perspective plot showing how the zone $\mathrm{B}$ is connected to the boundaries of the model which are held at $200 \mathrm{~m}$ head. 


\section{Extended Boundaries, Zone $B^{\prime}$}

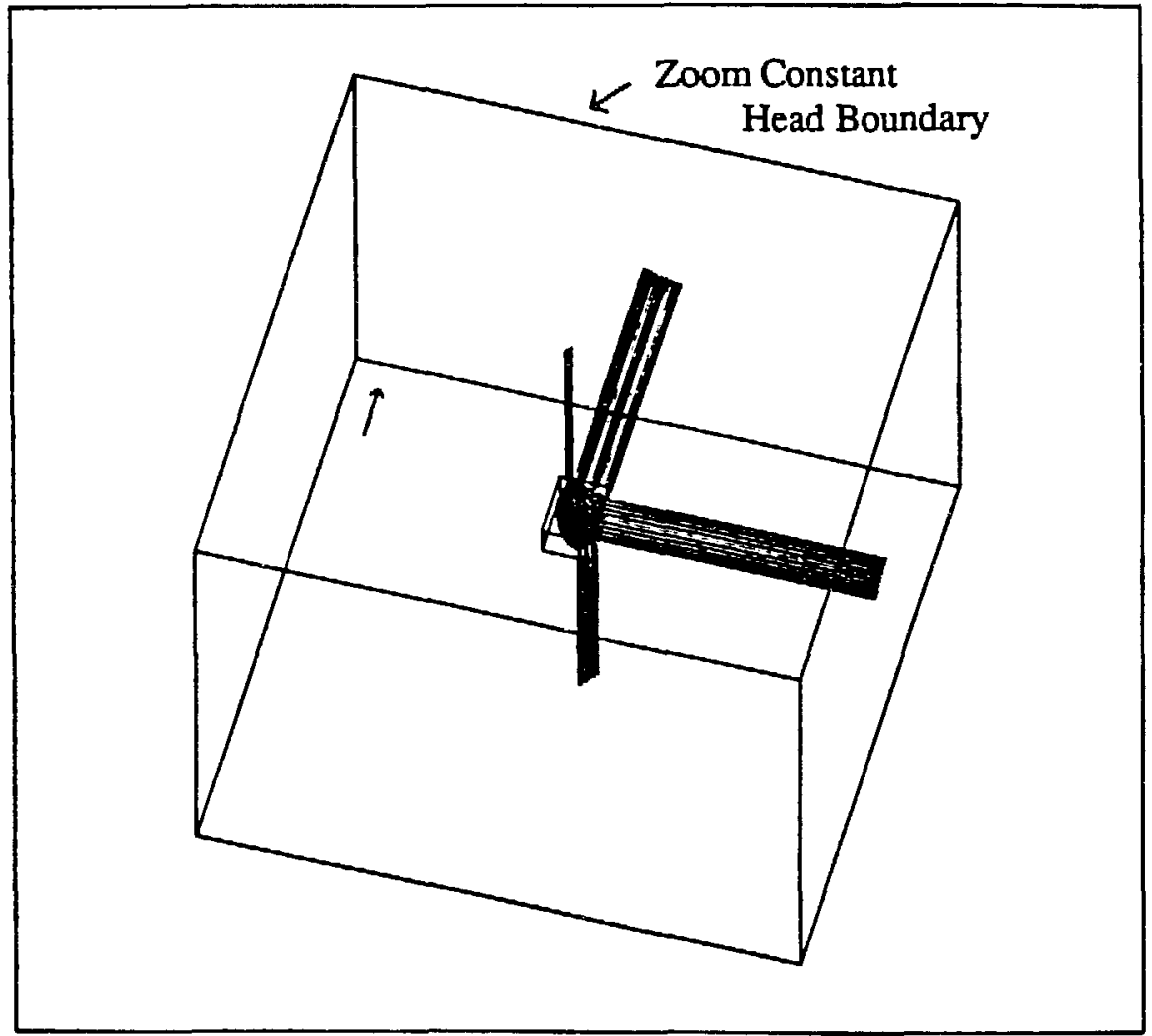

Figure 6.8c. Perspective plot showing how the zone $\mathrm{B}^{\prime}$ is connected to the boundaries of the model which are held at $200 \mathrm{~m}$ head. 


\section{Extended Boundaries, Zone C}

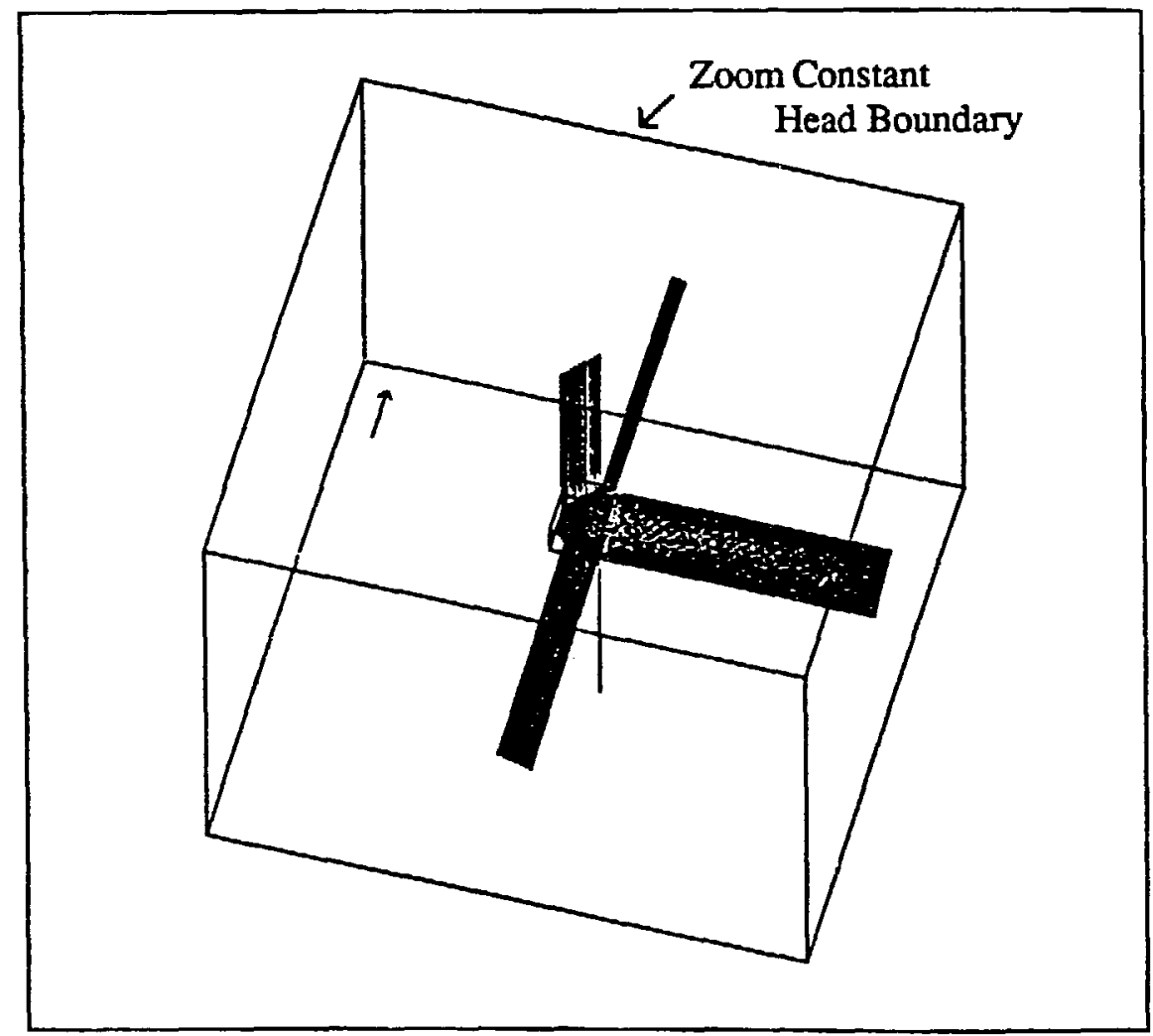

Figure 6.8d. Perspective plot showing how the zone $\mathrm{C}$ is connected to the boundaries of the model which are held at $200 \mathrm{~m}$ head. 


\section{Extended Boundaries, Zone Ha}

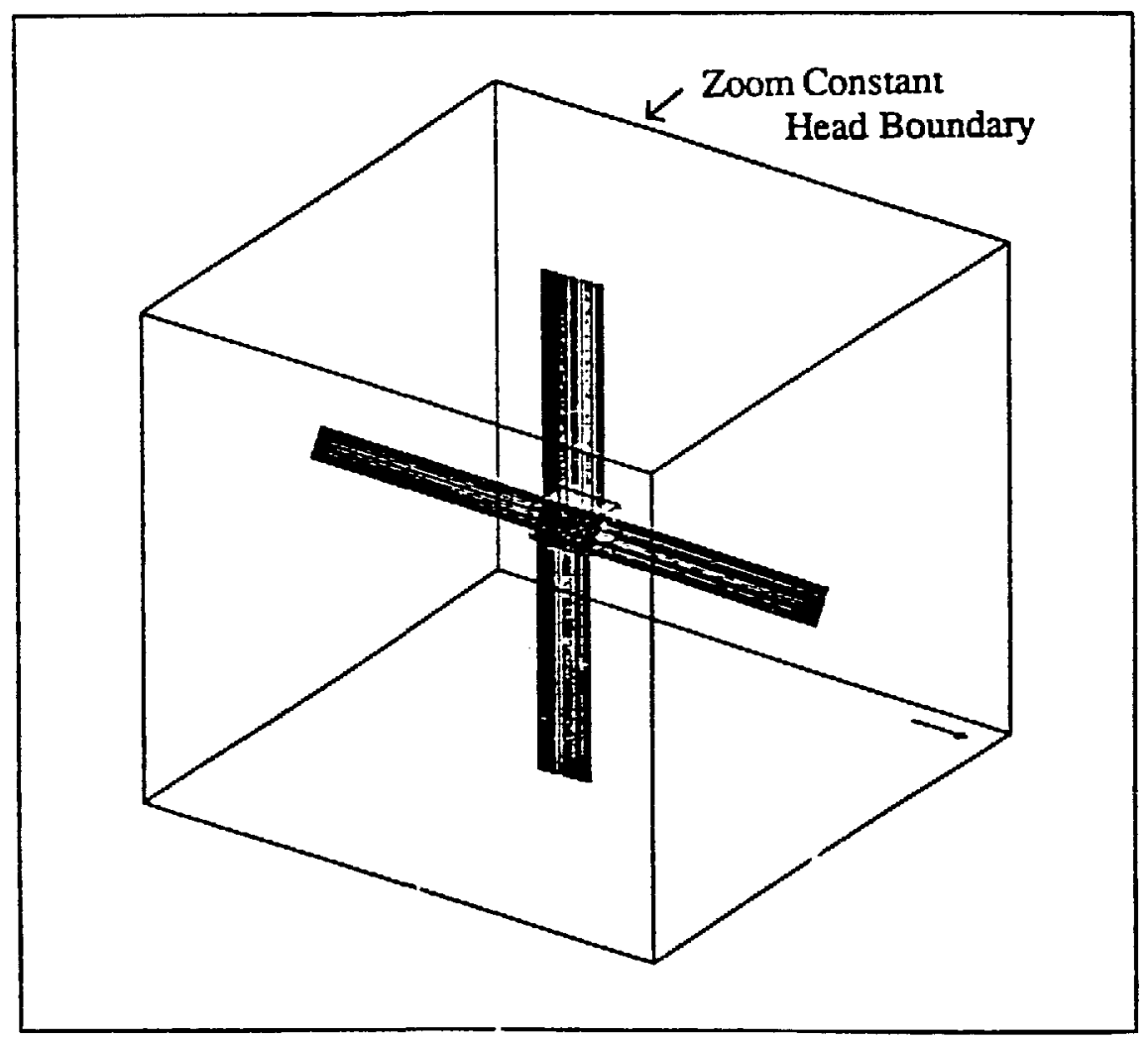

Figure 6.8e. Perspective plot showing how the zone Ha is connected to the boundaries of the model which are held at $200 \mathrm{~m}$ head. 


\section{Extended Boundaries, Zone $\mathrm{Hb}$}

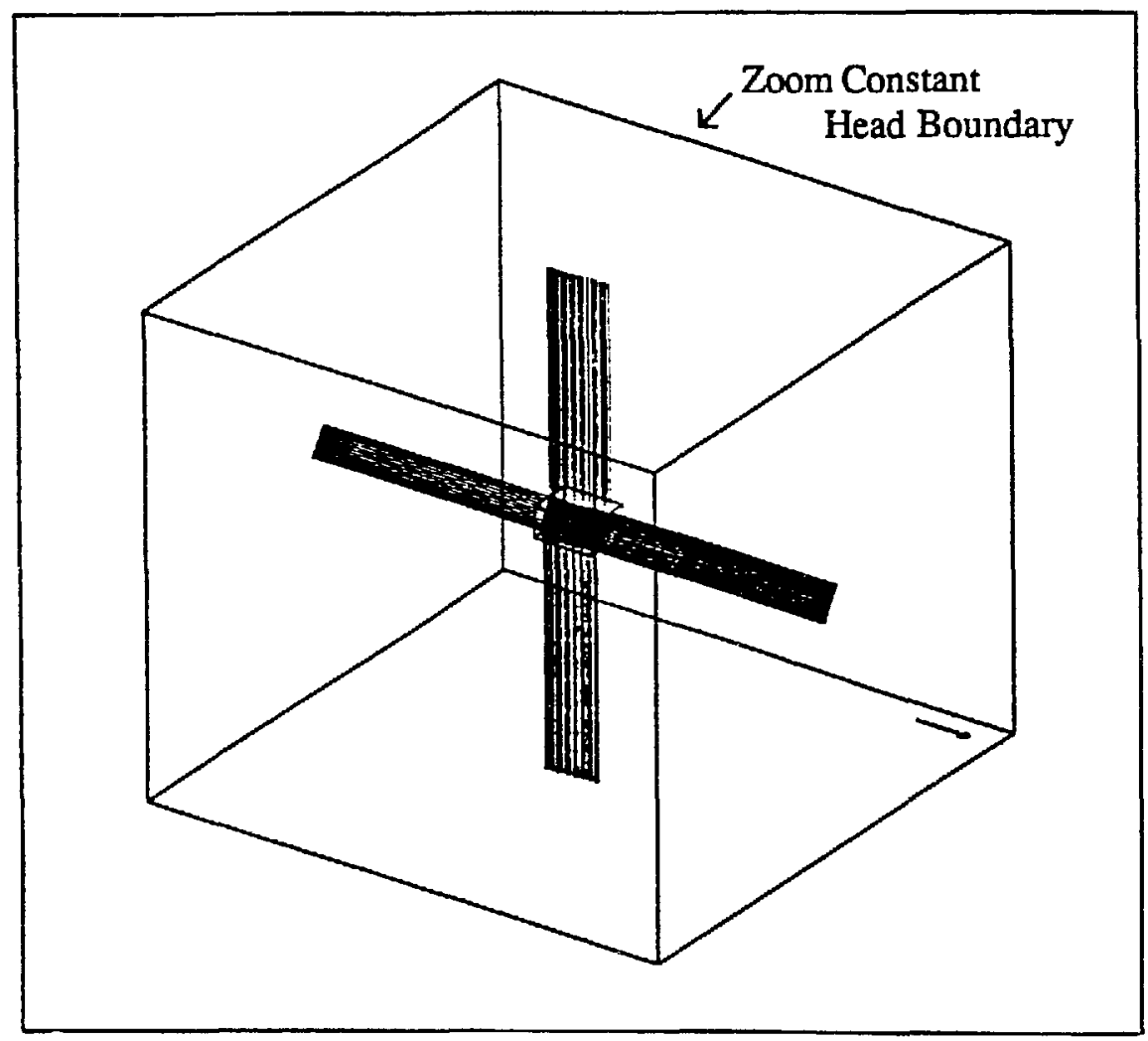

Figure 6.8f. Perspective plot showing how the zone $\mathrm{Hb}$ is connected to the boundaries of the model which are held at $200 \mathrm{~m}$ head. 


\section{Extended Boundaries, Zone I}

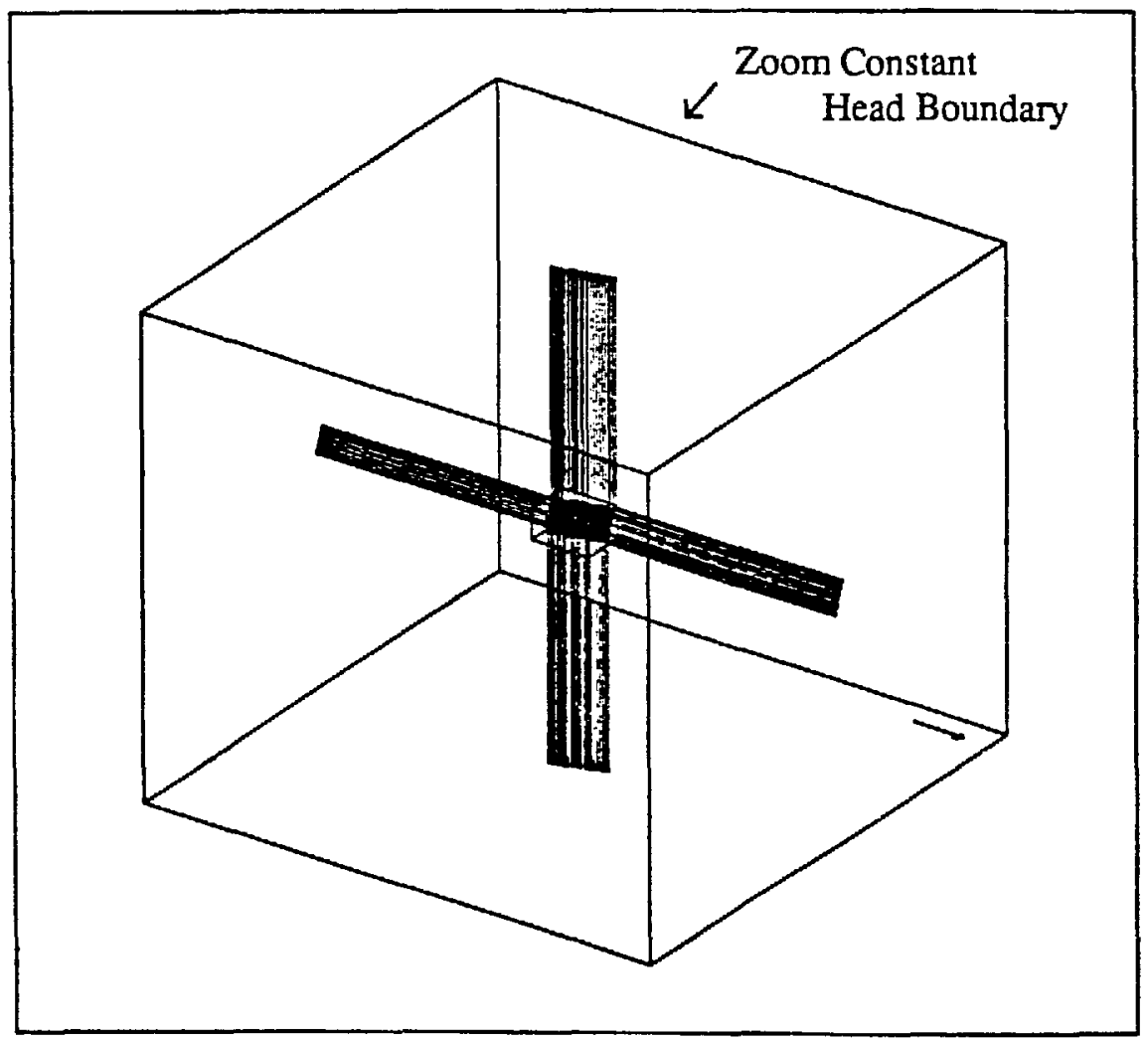

Figure 6.8g. Perspective plot showing how the zone I is connected to the boundaries of the model which are held at $200 \mathrm{~m}$ head. 
One remaining question is whether to include the drifts themselves in the model in order to incorporate their effect on the heads in the block. We decided against this as a first approximation because the heads in the SCV block very near the drifts are close to $200 \mathrm{~m}$. Thus we would have to surround the drifts with a low permeability zone or they would cause a much larger head drop in the model than is observed. Surrounding the drifts with a low permeability zone would be almost equivalent to not including them, so we left them out.

A modification of the energy function was defined for this case because there is some uncertainty about the boundary conditions. We are not very confident of the the inner and outer boundary conditions because local heterogeneities near W2 will cause the system to behave as if the boundary at W2 is not zero head. If there is a zone of low permeability around the well, the effective head is higher than zero and vice versa. We know much less about the outer boundary condition. The relative head structure observed away from these uncertainties is considered to contain more information. So, the inner and outer boundary conditions were shifted up or down such that we could obtain the minimum relative distance to the observed values. In this way, we are putting all the annealing emphasis on the pattern of the head values, not on the absolute value of the heads.

As a staring configuration we randomly removed $20 \%$ of the conductors within the block in order to enhance the speed of convergence to a low energy. We expect that annealing will remove many of the conductors in the template in order to match the observed behavior. Making "holes" a priori improves the speed of convergence because if we start with the full grid, the annealing process will tum off every conductor it examines at the early stage anyway. In the present case we chose to do random preconditioning and an arbitrary percentage of 20 instead.

\subsection{The Energy Versus Iteration Curve}

A temperature schedule was chosen as:

$$
T_{k+1}=T_{k} 0.7^{k}
$$

with

$$
T_{0}=0.1
$$

and the temperature was changed every 20 successful iterations. The annealing program was initiated and continued running for 931 iterations during a period of one week. At that time an inefficient algorithm in the program was detected which was causing the annealing procedure to occasionally retain the previous configuration unnecessarily. This is evidenced in Figure 6.9 between iteration 1 and 931 by the regions on plot where there are two values of energy plotted for the same iteration. At the same time, we found that the head in N3 had been left out of the energy calculation. Hence, the process was stopped and restarted with a refreshed temperature schedule, the current minimum energy fracture configuration, the error corrected, and the fourh well included. Recall from the discussion of annealing theory that a restart is justified because these configurations are random Markov Fields.

The added well sharply increased the energy level at this point (Figure 6.9, iteration 932). With the process correctly functioning the procedure continued for another five days until the temperature schedule was exhausted and the procedure terminated normally at iteration number 1813 . The energy at this iteration was 1.74 . To see if we could get the energy closer to zero, the annealing program was restarted with an 


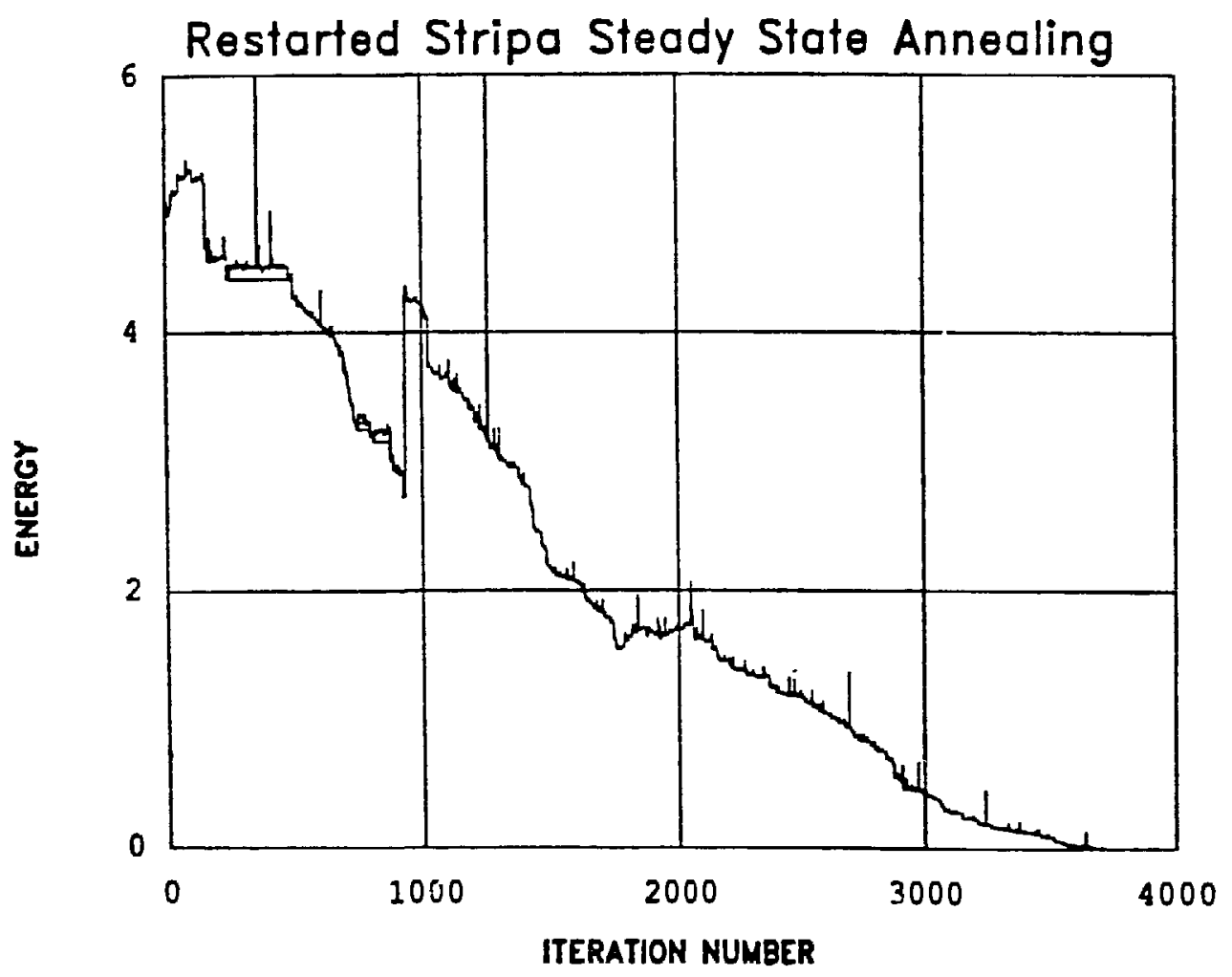

XBI 896-2395

Figure 6.9. Record of energy versus iteration for the SCV synthetic well test case. 
extended temperature schedule at the current configuration. Also, the annealing program was altered to increase the number of iterations at each temperature from 20 to 50. This change is reflected in the decreased slope of the receding energy function. The process was stopped at iteration number 3749 and energy equal to 0.005661 .

Figures 6.10a through $\mathrm{g}$ show the configuration of the channels in each zone. Each figure shows the complete set of possible channels, the initial configuration of channels at the start of annealing, and the configuration of channels at the last iteration. One can see that the percentage of elements did not change much from the initial configuration to the final one. Davey et al (1990) have studied this problem with synthetic examples to see if the starting percentage controls the final percentage. For the cases studied, their results show that annealing results are independent of the starting percentage. For the SCV case, we surmise that the annealing case did not contain enough information to warrant changing the percentage of elements. In other words, the chance of removing elements was equal to the chance of replacing elements because there was no information to indicate otherwise.

There are only slight indications that the annealing routine was beginning to find conductor pattems. On zone A, we see that connections between $\mathrm{N} 4$ and $\mathrm{N} 3$ have become a little sparser after annealing which reflects the fact that zone $A$ responded to $W 2$ in N3 but not in N4. Similar observations are that "holes" developed in zone B between $\mathrm{N} 2$ and $\mathrm{Ha}$, and $\mathrm{W} 2$ and $\mathrm{Hb}$, whereas the connection between $\mathrm{N} 3$ and I remained about the same. In B' we see W1 becoming more connected to W2, but not much other change. In zone $\mathrm{C}$, connection between $\mathrm{I}$ and $\mathrm{Hb}$ increased. However, none of these changes are very striking and we suspect again that we simply do not yet have enough information to discem the channel pattem.

Table 6.2 gives the heads that were calculated with the final configuration resulting from annealing. We see that the annealing routine has managed to match the observed heads very well. The other values of head can be compared to those measured when the D-holes are brought to near zero pressure. However, there is a great deal of uncertainty about the boundary conditions and these predictions are directly related to the applied boundary conditions.

\subsection{Calibration to W2 Flow (Shifting)}

At the end of the annealing process, we have determined several configurations of conductors within the zones all of which result in matching the observed head data extremely well. The match has been achieved solely by arranging the conductors. As all the channels have the same conductance, $k_{A}$, any value of $k_{A}$ will resuit in the same head distribution. So, at this point we must calibrate the model such that it will predict the correct value of flow from W2. To do this, we use the annealed model to calculate the flow from $\mathrm{W} 2, \mathrm{QW} 2 \mathrm{~A}$ with all conductances equal to $\mathbf{k}_{\mathrm{A}}$. Then we take the ratio of measured flow to calculated flow to find the conductance of all the channels, kW2 which would produce the correct amount of flow into W2 $(\mathrm{QW} 2 \mathrm{M})$.

$$
k W 2=k_{A} \cdot \frac{Q W 2_{M}}{Q W 2_{A}}
$$



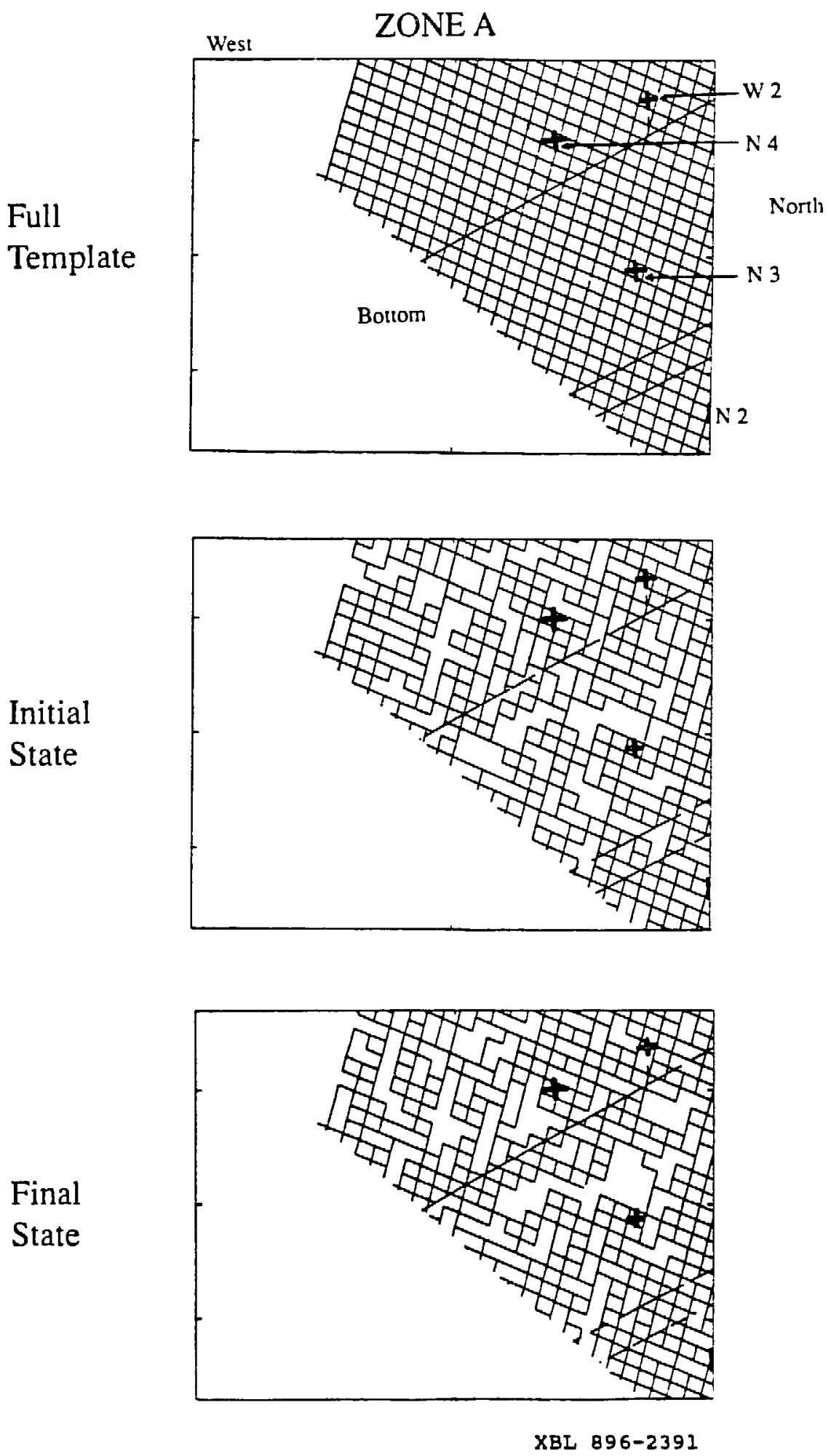

Figure 6.10a. Grid elements in zone A. The top is the full template, the middle is the initial configuration, and the bottom is the final configuration after annealing. 

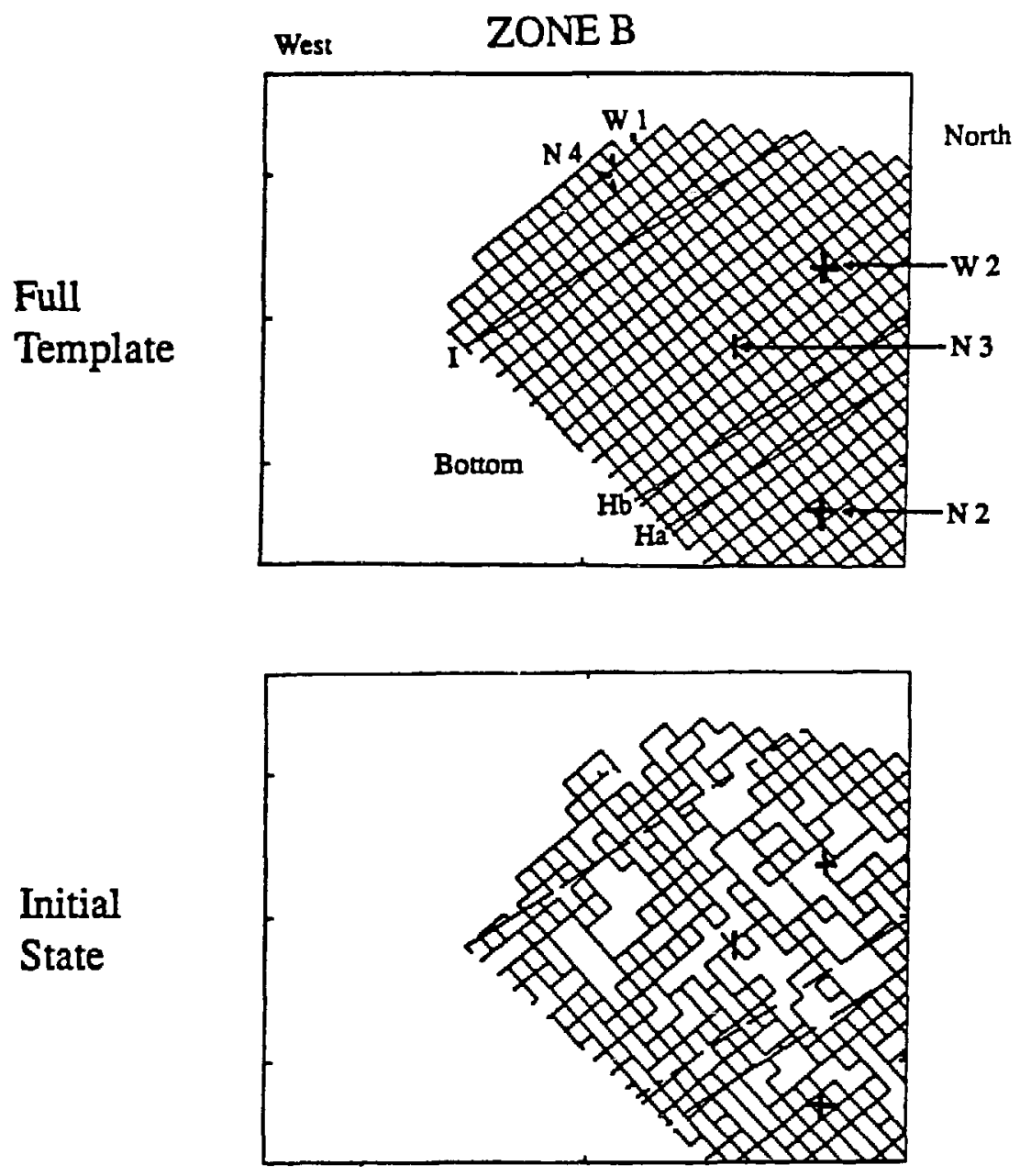

Final

State

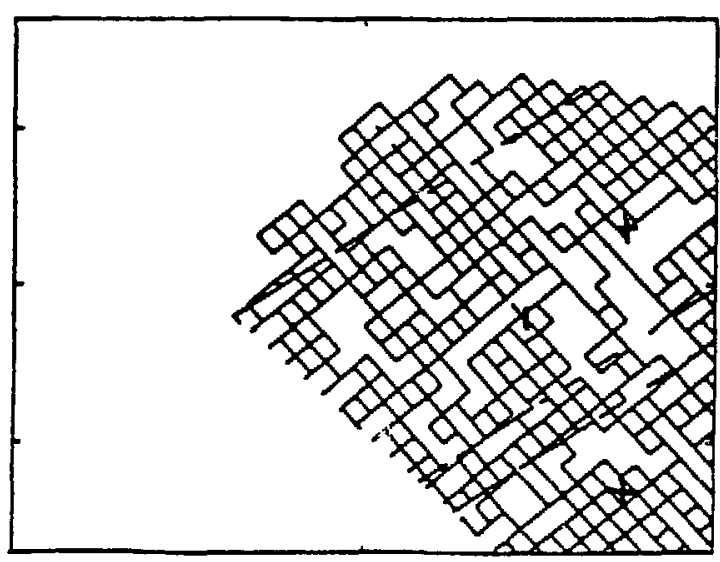

Figure 6.10b. Grid elements in zone B. The top is the full template, the middle is the initial configuration, and the bottom is the final configuration after annealing. 

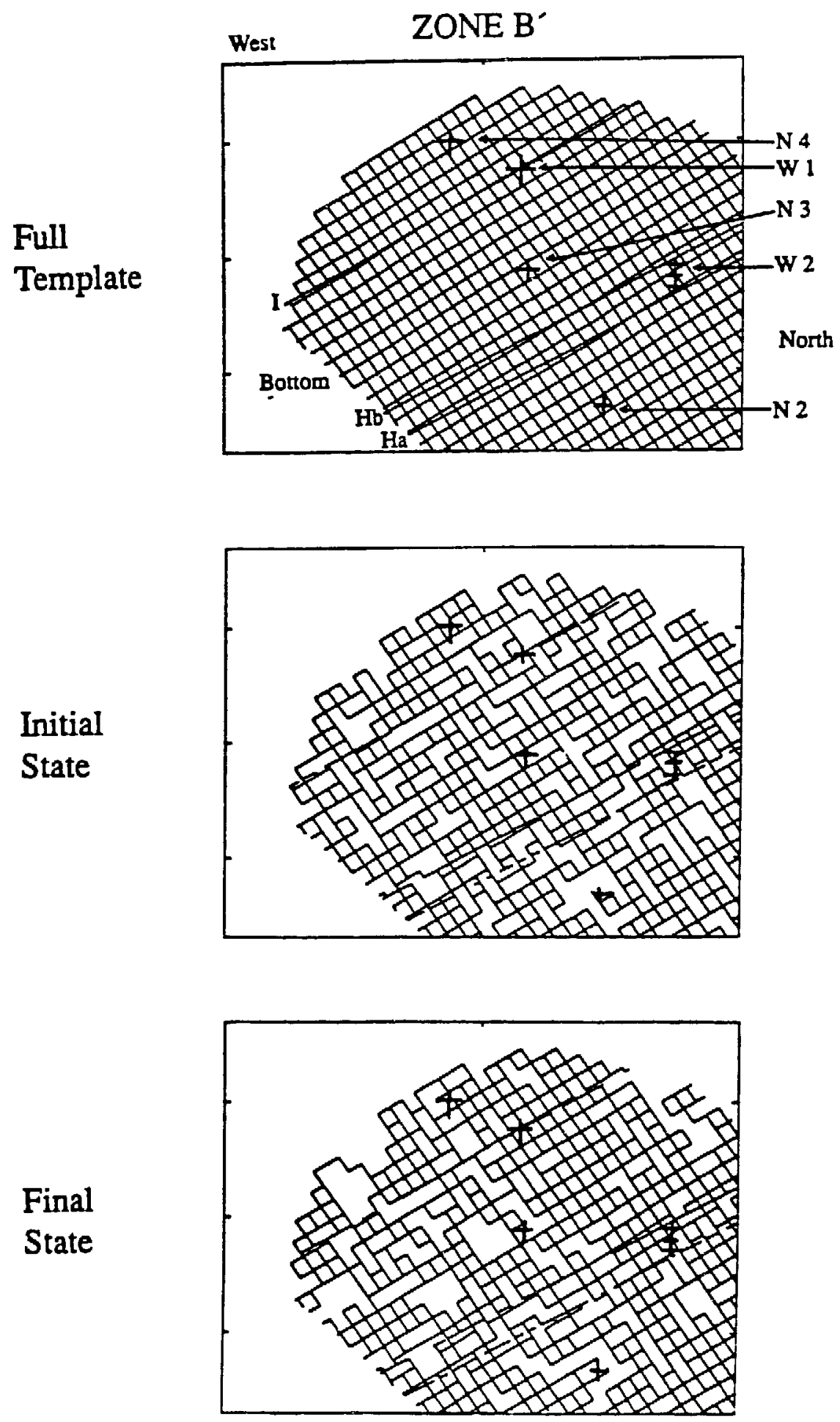

Figure 6.10c. Grid elements in zone B'. The top is the full template, the middle is the initial configuration, and the bottom is the final configuration after annealing. 
Full

Template

ZONE C

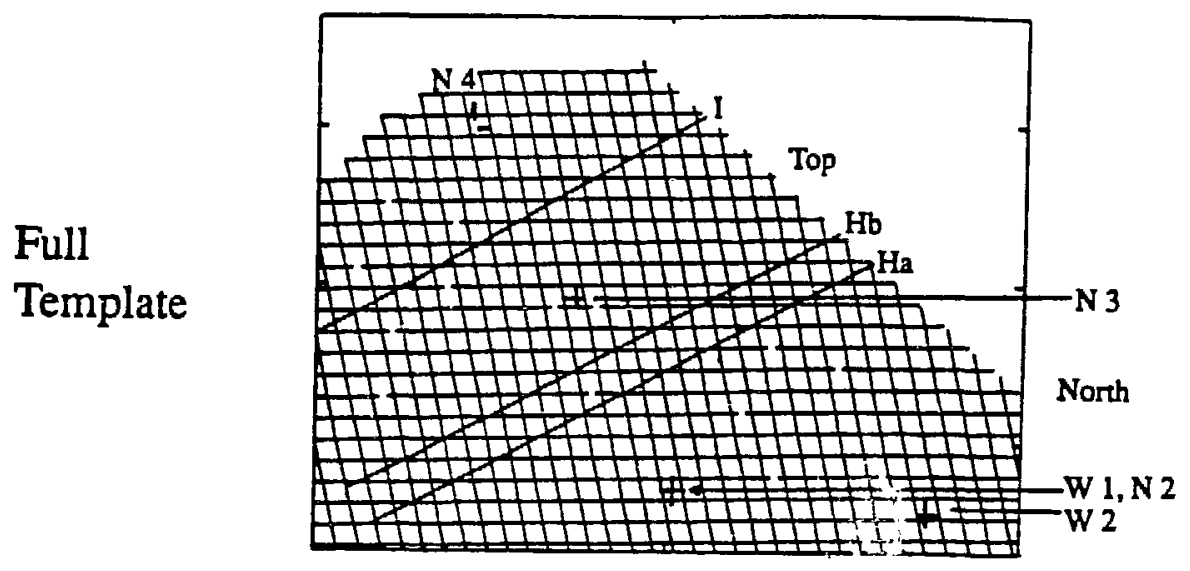

Initial

State

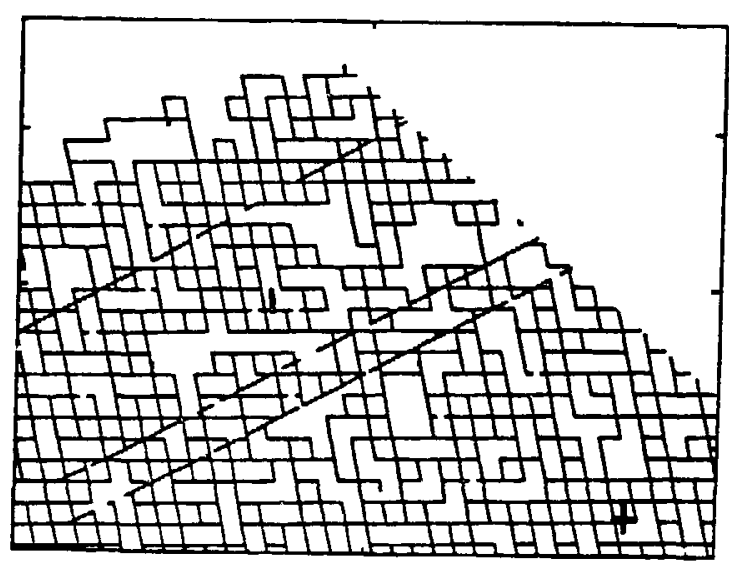

Final

State

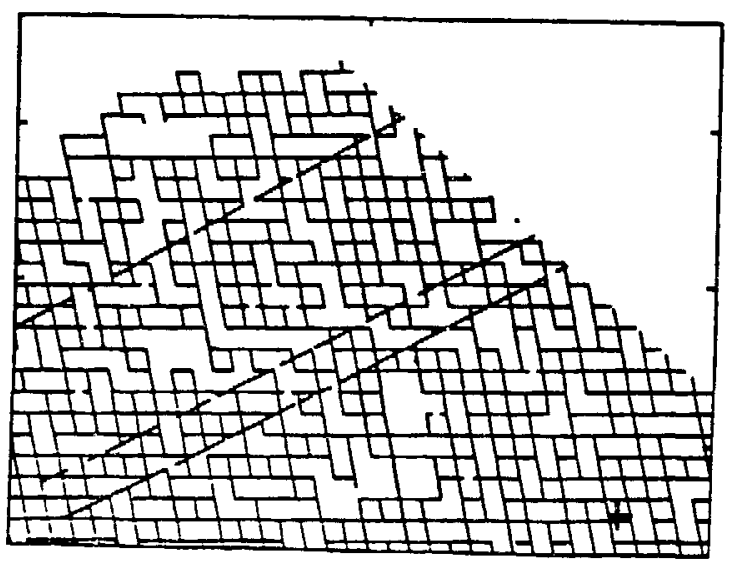

Figure 6.10d. Grid elements in zone $C$. The top is the full template, the middle is the initial configuration, and the bottom is the final configuration after annealing. 
Full

Template

ZONE Ha

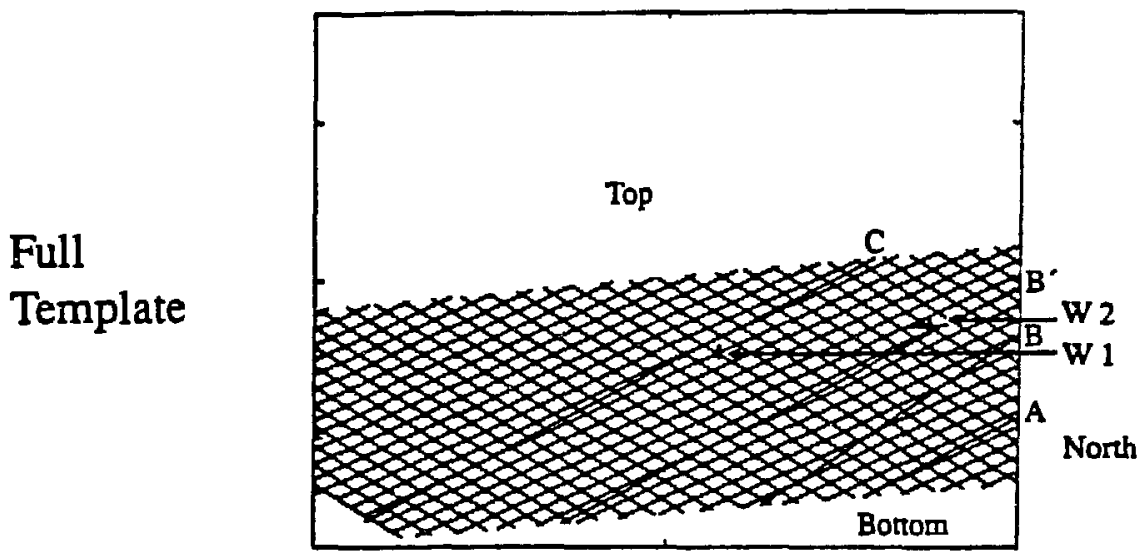

Initial

State

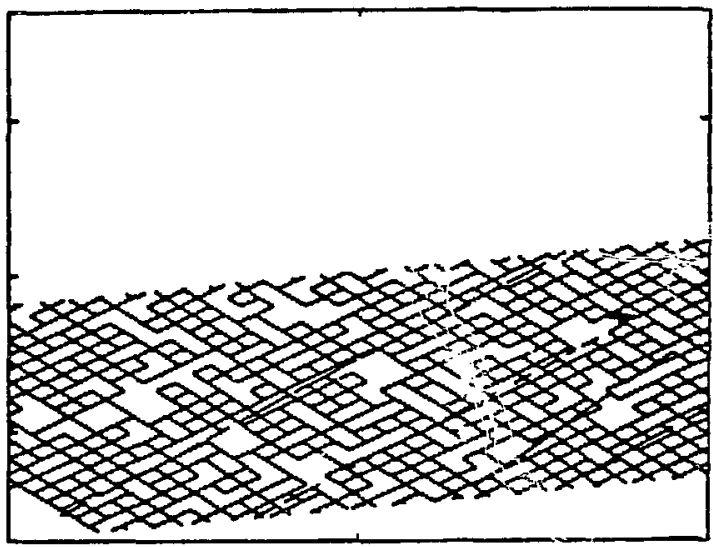

Final

State

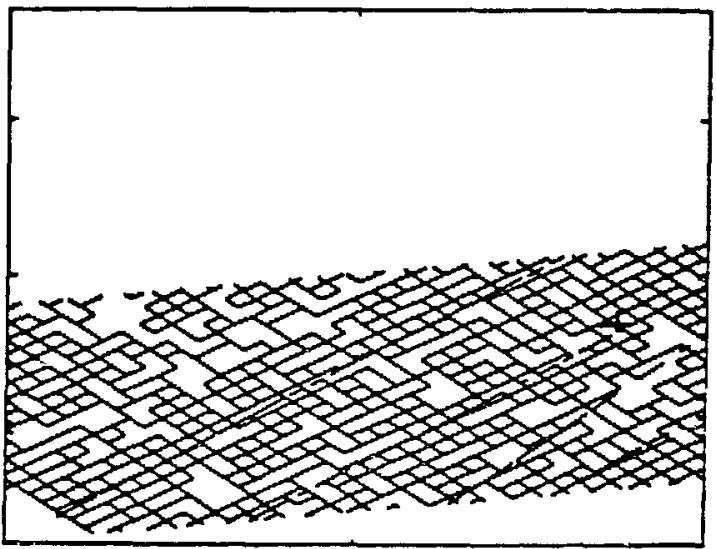

Figure 6.10e. Grid elements in zone Ha. The top is the full template, the middle is the initial configuration, and the bottom is the final configuration after annealing. 
ZONE Hb

Full

Template

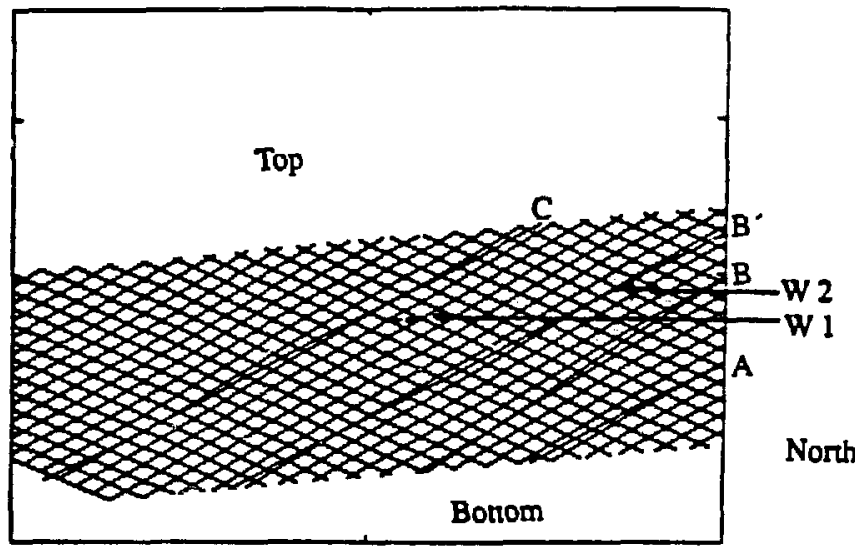

Initial

State

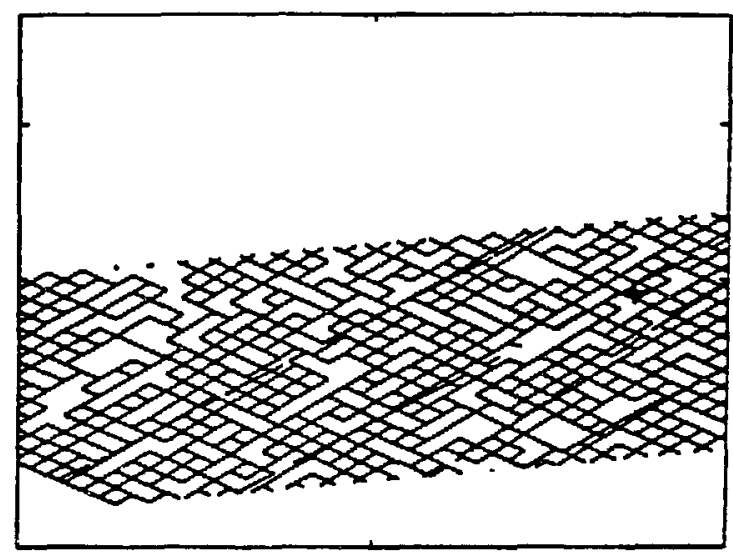

Final

State

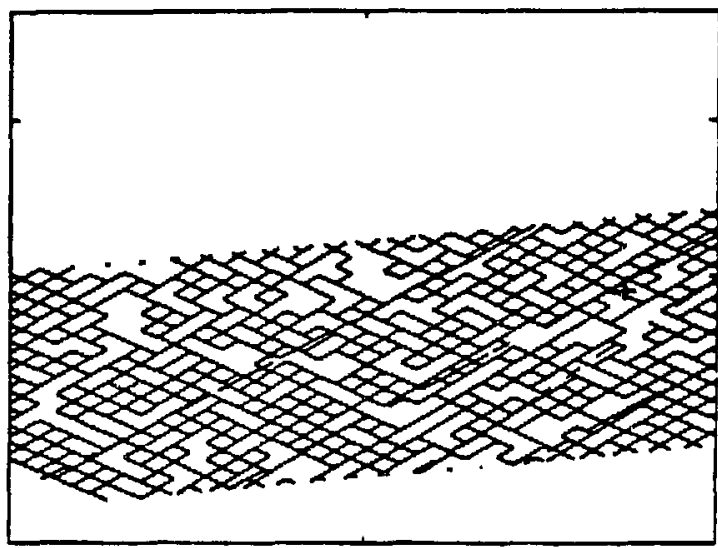

Figure 6.10f. Grid elements in zone $\mathrm{Hb}$. The top is the full template, the middle is the initial configuration, and the bottom is the final configuration after annealing. 


\section{ZONE I}

Full

Template

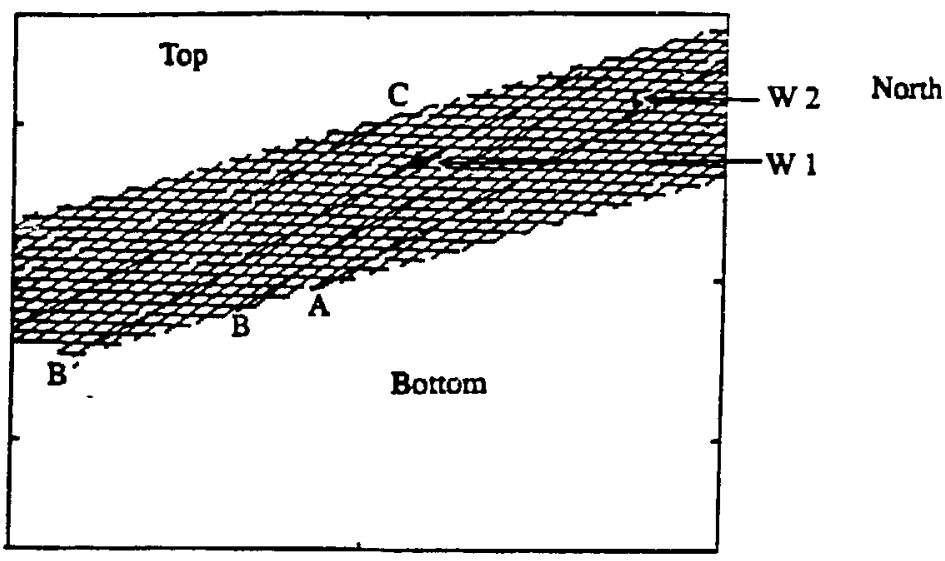

Initial

State

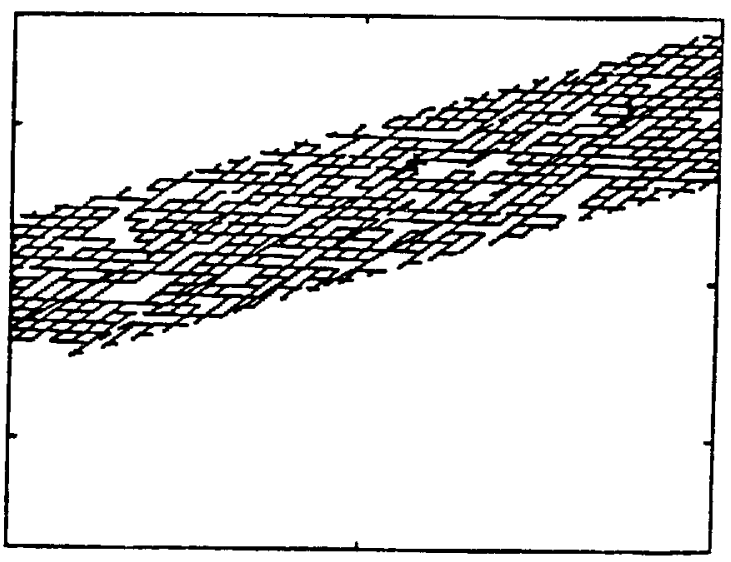

Final

State

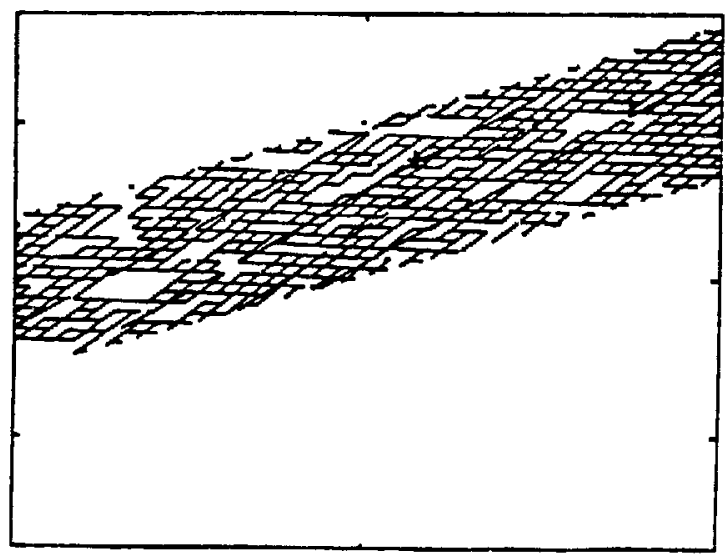

Figure 6.10g. Grid eiements in zone $\mathrm{I}$. The top is the full template, the middle is the initial configuration, and the bottom is the final configuration after annealing. 
Table 6.2. Annealing results at the final iteration $=3749$

\begin{tabular}{|c|c|c|c|}
\hline Hole & Zone & Observed Heads & Predicted Heads \\
\hline \hline N2 & B,B $^{\prime}, \mathrm{C}$ & 90 & 90 \\
N3 & $\mathrm{A}^{\prime} \mathrm{B}$ & 80 & 79 \\
& $\mathrm{~B}^{\prime}$ & none & 65 \\
& $\mathrm{C}$ & none & 65 \\
N4 & $\mathrm{B}^{\prime}$ & 55 & 55 \\
& $\mathrm{C}$ & none & 83 \\
& $\mathrm{~B}$ & none & 49 \\
& $\mathrm{~A}$ & none & 49 \\
W1 & $\mathrm{H}_{\mathrm{a}}, \mathrm{C}$ & 65 & 65 \\
& $\mathrm{H}_{\mathrm{b}}$ & none & 65 \\
& $\mathrm{~B}^{\prime}$ & none & 65 \\
& $\mathrm{~B}^{\prime}$ & none & 65 \\
\hline
\end{tabular}

For the final configuration of the model, the value of $Q W 2 A$ is $3.14 \mathrm{~m}^{3} / \mathrm{s}$, and $k_{A}$ is 0.42 $\mathrm{m}^{3} / \mathrm{s}$, so for the case of $\mathrm{QW} 2_{\mathrm{M}}=10 \mathrm{~L} / \mathrm{min}=1.67 \times 10^{-2} \mathrm{~m}^{3} / \mathrm{s}$, we require that $\mathrm{kW} 2=2.2 \times 10^{-5} \mathrm{~m}^{3} / \mathrm{s}$.

Now we rearrange the numerical model, closing the hole W2, and adding the open Dholes and calculating the outflow frorn the D-holes. We repeat this for seven different configurations of the model. The resulting calculations of inflow to the $D$-holes are given in Table 6.3.

Table 6.3. Inflows to the D-Holes Predicted for Seven Different Configurations of the Model.

\begin{tabular}{|c|c|c|c|}
\hline $\begin{array}{c}\text { Rank of } \\
\text { Likelihood }\end{array}$ & Iteration & Energy & $\begin{array}{c}\text { Inflow to the } \\
\text { D-holes (1/min) }\end{array}$ \\
\hline 1 & 3747 & 0.005 & 8.9 \\
2 & 3568 & 0.096 & 5.0 \\
3 & 2910 & 0.63 & 9.1 \\
4 & 2498 & 1.32 & 8.9 \\
5 & 949 & 2.94 & 8.8 \\
6 & 847 & 3.13 & 8.8 \\
7 & 300 & 4.62 & 8.9 \\
\hline
\end{tabular}

In this case, the prediction of inflow is relatively insensitive to the energy, which means it is insensitive to the pattem. Again, this indicates that the annealing case did not contain enough information to influence the results. We will have to wait until cross-hole tests are available to see if we can leam something about fracture pattem at Stripa through annealing.

What does influence the prediction of inflow to the D-holes is the measurement of flow from the W2 bole. In fact, by the scaling equation above, the flow into the D-holes is 
directy proportional to the flow from W2. This indicates that the prediction of D-hole inflow is extremely sensibive to the measurement of ficws. The calculations given in Table 6.0 depend on a single measurement of flow, and we suspect that this measurement is anomalous in that the transmissivity in W2 is much higher than the other holes. This points out that other flow data available for the SCV block would be very useful in modifying this prediction and this is discussed below.

\subsection{Modification of the Prediction Using Other Inflows}

One further set of data is available to aid in the prediction of inflow to the D-holes. This data was also collected on an ad hoc basis by RGS (D. Holmes, personal communication) and consists of measurements of outfiu ws from the other $\mathrm{N}$ - and W-holes after they had been left open for periods of time. Table 6.4 gives this data.

Table 6.4. Open Fiole Infiows to $\mathrm{N}$ - and W-holes

\begin{tabular}{|c|c|l|}
\hline Hole & Flow (l/min) & \multicolumn{1}{|c|}{ Comment } \\
\hline \hline N2 & 0.60 & Measured flow after 6 hours open \\
N3 & 0.45 & $\begin{array}{l}\text { Measured flow after 4 hours open } \\
\text { N4 }\end{array}$ \\
W1 & 1.55 & $\begin{array}{l}\text { Measured flow after 2 hours open } \\
\text { Measured flow after 2 days of } \\
\text { chemical sampling } \\
\text { W2 }\end{array}$ \\
\hline
\end{tabular}

First, we note in Table 6.4 that the measured inflow to W2 is $121 / \mathrm{min}$. Our best estimate of the steady flow to W2 in $10 \mathrm{l} / \mathrm{min}$, or about $83 \%$ of that in Table 6.4 . This is because the values in Table 6.4 do not yet reflect steady flow. To correct for this, each of the measurements of flow is reduced by $17 \%$. We call these flows $Q_{i M}$ where $i$ stands for the holes, N2, N3, N4, W1, and W2.

Now, we can use the final annealed configuration of channels with conductance, $k_{A}$, to calculate the inflow into each of these holes, $\mathrm{Q}_{\mathrm{iA}}$. To do this we simply close W2 by making the nodes at W2 intemal nodes, then sequentially open each of the other holes by assigning their nodes zero head. In each case we calibrate the channel conductance in the same manner as previously described such that the model correctly predicts $Q_{i M}$ :

$$
\mathbf{k}_{\mathrm{i}}=\frac{\mathrm{Q}_{\mathrm{iM}}}{\mathbf{Q}_{\mathrm{iA}}} \mathbf{k}_{\mathbf{A}}
$$

This results in five different predictions of channel conductance, which in tum results in five different predictions of D-hole inflow. These predictions are given below in Table 6.5.

In summary, our prediction of inflow to the D-holes has mean $3.151 / \mathrm{min}$ and a coefficient of variation (standard deviation divided by the mean) equal to about one. To reinforce the point that the resulting prediction is dominated by the flow measurements, we note that the mean and standard deviation of the predicted inflow is close to the mean and standard deviation of the adjusted observed flows from the five hoies. We do not know the form of the inflow distribution function, but if we make a guess that 
inflows are distributed log-normally, then we can calculate that the flow will be between 0.44 and 11.4 with $95 \%$ confidence.

Table 6.5. Predictions of D-Hole Inflow Based on Annealing and Measured $\mathrm{N}$ - and W-Hole Inflows.

\begin{tabular}{|c|c|c|c|c|}
\hline Hole & $\begin{array}{c}\text { Measured } \\
\text { Flow } \\
(\mathrm{l} / \mathrm{min})\end{array}$ & $\begin{array}{c}\text { Adjusted } \\
\text { Flow } \\
(\mathrm{l} / \mathrm{min})\end{array}$ & $\begin{array}{c}\mathrm{k}_{\mathrm{l}} / \mathrm{k}_{\mathrm{A}} \\
\left(\frac{\mathrm{l} / \mathrm{m}}{\mathrm{m}^{3} / \mathrm{S}}\right)\end{array}$ & $\begin{array}{c}\text { Predicted } \\
\text { D-hole inflow } \\
(\mathrm{L} / \mathrm{min})\end{array}$ \\
\hline N2 & 0.60 & 0.50 & 0.16 & 1.3 \\
N3 & 0.45 & 0.37 & 0.12 & 0.6 \\
N4 & 2.6 & 2.1 & 0.67 & 3.4 \\
W1 & 1.3 & 1.1 & 0.35 & 1.3 \\
W2 & 12.0 & 10.0 & 3.2 & 8.9 \\
\hline \hline Mean & 3.4 & 2.8 & .89 & 3.1 \\
\hline Standard & & & & 3.1 \\
Deviation & 4.9 & 4.1 & 1.3 & 3.1 \\
\hline
\end{tabular}

\subsection{Estimation of Prediction Error}

Recall that to calculate prediction error, we need more than one test, say $\mathbf{N}$ tests. These $\mathrm{N}$ tests would each consist of a measured flow rate plus measured head rer ronses. Then we could anneal the model $N$ times, each time leaving out the data fio. . one of the tests. With each model we would predict the flow for the test that was left out, calculate the difference between the observed flow and the predicted flow, take the root mean square of these differences to get the estimate of prediction error.

In our case, we only have draw-down data from one test, but we have five different measurements of inflow. So, to make an estimate of prediction error, we divide the data into two types, head data and flow data. We anneal with the head data alone to get a channel configuration. Then we develop five different models by calculating channel conductance with only four of the inflows at a time. Thus, we average the conductances, $k_{i}$, from Table 6.5 leaving out one value (the ith value) at a time to get $\left\langle k_{i 0}\right\rangle$. Then we use this value to compute the flow into the hole we left out of the average. The root mean square of the differences between predicted and observed flux for each case is the estimate of prediction error. These values are given below in Table 6.6.

\subsection{Estimate of Distribution of Flow Between D-Holes}

The total inflow can be partioned along the D-holes into the contribution expected from each zone. There is currently no data on the head distribution near the D-holes. As a result, we could not anneal to find the pattem of conductors intersecting the six holes. So, in order to estimate how flow would be distributed between the six D-holes, we made a bootstrapped estimate. These results based on the final iteration are given below in Table 6.7 . 
Table 6.6. Prediction Error

\begin{tabular}{|c|c|c|c|c|}
\hline Hole left out & $\left\langle\mathrm{k}_{\mathrm{io}}\right\rangle$ & $\begin{array}{l}\text { Predicted } \\
Q_{i}(1 / m i n)\end{array}$ & $\begin{array}{l}\text { Observed } \\
\mathrm{Q},(\mathrm{l} / \mathrm{min})\end{array}$ & $\begin{array}{c}\text { Error } \\
(1 / \mathrm{min})\end{array}$ \\
\hline $\begin{array}{l}\text { N2 } \\
\text { N3 } \\
\text { N4 } \\
\text { W1 } \\
\text { W2 }\end{array}$ & $\begin{array}{l}1.1 \\
1.1 \\
.96 \\
1.0 \\
.33\end{array}$ & $\begin{array}{l}3.4 \\
3.4 \\
3.0 \\
3.3 \\
1.0\end{array}$ & $\begin{array}{l}.5 \\
.37 \\
2.1 \\
1.1 \\
10 .\end{array}$ & $\begin{array}{c}2.9 \\
3.0 \\
.90 \\
2.2 \\
9 .\end{array}$ \\
\hline \multicolumn{4}{|c|}{ Estimated Prediction Error } & 4.6 \\
\hline
\end{tabular}

Table 6.7. Partition of the Expected Flow into the D-Holes from Each Zones

\begin{tabular}{|c|c|}
\hline Zone & Expected inflow (L/min) \\
\hline \hline A & No intersection \\
B & 0.61 \\
$\mathrm{~B}^{\cdot}$ & 0.49 \\
$\mathrm{C}$ & 0.44 \\
$\mathrm{H}_{\mathbf{2}}$ & 0.44 \\
$\mathrm{H}_{\mathrm{b}}$ & 0.64 \\
$\mathrm{I}$ & 0.41 \\
\hline Mean & 0.51 \\
Standard deviation & 0.097 \\
\hline
\end{tabular}

A bootstrapped estimate is one where an empirical distribution of the variation is obtained from other parts of the block. This distribution is resampled many times to provide examples of possible variation. The samples drawn from this empirical distribution are then used to calculate estimates of the mean and variance of flow into a $D$ hole. The following assumptions were made:

1. All six D-holes intersect the same fractured zones.

2. The main cause of local variation in the flow into the $D$-holes is variation in the hydraulic conductivity of the fracture zones.

3. The distribution of the local conductivity in the fracture zones can be estimated using the measured distribution of the ten highest conductivities in each borehole. This distribution is called the "empirical" distribution (Figure 6. 11, top).

4. The middle D-hole should have almost no flow.

5. The sum of the flows into the five outside D-holes is equal to the total flow predicted in the annealing results. Further, we assume that flow is proportioned between the boreholes in the same ratio that transmissivity is proportioned.

We calculated a bootstrap estimate for the variation between the holes (Figure 6.11, bottom) as follows: 
(a)

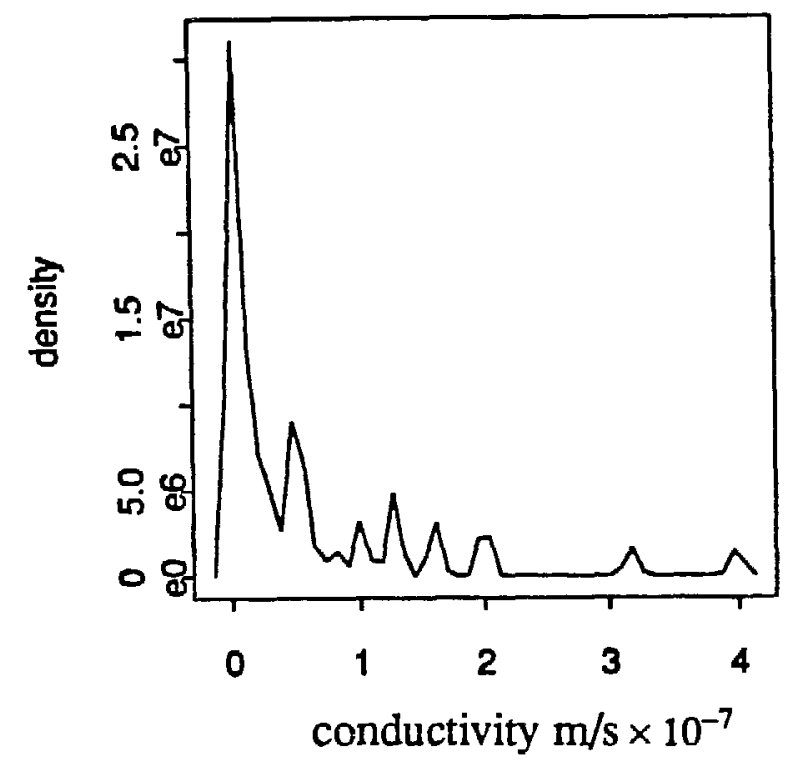

(b)

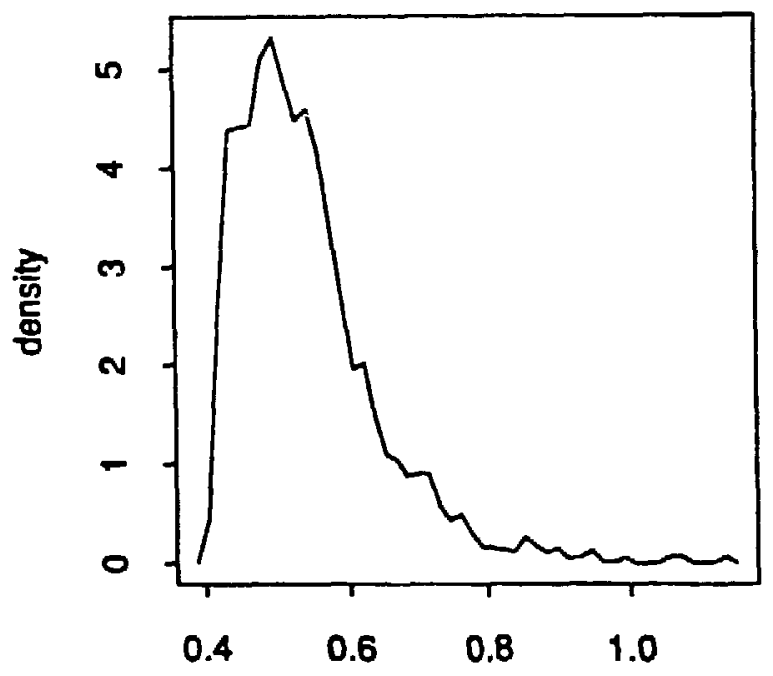

flow variance $(l / \mathrm{min})^{2}$

Figure 6.11. The distributions used to make an estimate of the variation in flow between the D-holes. (a) Empirical density of hydraulic conductivity from the 10 most permeable zones in the $\mathrm{N}$ and $\mathrm{W}$ boreholes. (b) Estimated density of flow variance between the individual D-holes based on bootstrapped sampling of the empirical distribution of conductivities and the estimate of total D-hole flow. 
1. The empirical hydraulic conductivity distribution for the fracture zones was used to randomly assign a transmissivity value to each of five $D$-holes.

2. The sum of all five transmissivities was found and used to calculate the proportion of flow for each borehole, using:

$$
\begin{aligned}
\mathbf{f}(l) & =\mathrm{D}_{\text {flow }} \cdot \mathrm{h}(i) / \sum \mathrm{h}(i) \\
& =\mathrm{D}_{\text {flow }} \cdot \mathrm{w}(i)
\end{aligned}
$$

where

$$
\begin{aligned}
& f(i)=\text { flow in the } i^{\text {th }} \text { D-hole } \\
& h(i)=\text { the hydraulic conductivity for the } i^{\text {th }} \text { D-hole } \\
& w(i)=\text { the fraction of conductivity for the } i^{\text {th }} \text { D-hole } \\
& D_{\text {flow }}=\text { flow into all the D-holes }
\end{aligned}
$$

3. Steps (1) and (2) are repeated 1000 times.

4. We estimate the mean and variance of the five weights, w(i).

5. The 1000 means and variances are averaged to find the bootstrap estimates of the true $w(i)$. We found a mean of 0.20 and a variance of 0.0089 .

6. The mean and variance of the flow can be calculated using our estimates for the mean and variance of the weights and the total flow into the D-holes as follows. The total flow into the D-holes was estimated using the network obtained by annealing (Table 6.5). Recall that we obtained five different estimates of total flow into the D-holes, $Q_{j}$, each based on a the outflow from one of the $N$ - or W-holes. If we assume that each estimate of total flow is equally likely, we can calculate a mean and variance for the total D-hole flow. The mean and variance of the flow for each $D$-hole is easily calculated if we assume independence between the normalized hydraulic conductivity and the total flow.

$$
\mathrm{E}[\mathrm{f}(i)]=\mathrm{E}[\mathrm{w}(\boldsymbol{i})] \cdot \mathrm{E}\left[D_{\text {how }}\right]
$$

Let

$$
\begin{aligned}
& X=D_{\text {flow }} \\
& E(X)=\mu_{X}
\end{aligned}
$$

and

$$
\begin{gathered}
Y=w(i) \\
E(Y)=\mu_{Y}
\end{gathered}
$$

Then

$$
\begin{aligned}
\operatorname{Var}[f(i)] & =E\left(X Y-\mu_{X} \mu_{Y}\right)\left(X Y-\mu_{X} \mu_{Y}\right) \\
& =E\left(X^{2} Y^{2}-\mu_{X}{ }^{2} \mu_{Y}{ }^{2}\right)
\end{aligned}
$$

Using our five values for the total D-hole flow $(1.25,0.6,3.4,1.3$, and $9.2 \mathrm{l} / \mathrm{min})$ we can find a mean (3.15) and variance (12.55) for total D-hole flow. By also using the boot strapped sampling of the empirical distribution of the top ten zone's transmissivity, we can calculate the variance of flow between the D-holes. The estimated mean and 
variance of the individual D-hole flows is

$$
\begin{gathered}
E[f(i)]=(0.20) \cdot(3.15)=0.63 \\
\operatorname{Var}[f(i)]=0.94-0.39=0.55
\end{gathered}
$$

This is a fairly large variance, with coefficient of variation equal to nearly one. This means that one of the D-holes could easily account for at least half of the total flow into all the D-holes.

The variance estimate may not be very good. The estimate is based on a small number of measured inflows, and the assumption of independence between the normalized conductivity and the total D-hole flow may not be that close to the truth. The actual variance may be higher or lower than our estimate. 


\section{SUMMARY OF RESULTS AND CONCLUSIONS}

Based on the preliminary data available at this time, the inflow predicted for the $D$ holes is $3.15 \mathrm{l} / \mathrm{m}$ with a prediction error equal to $4.6 \mathrm{l} / \mathrm{m}$. The coefficient of variation for the individual flows to the D-holes is about 1.0. The results are most sensitive to the measurements of inflow. We think that this is particularly so due to the fact that the annealing case was so limited and especially that only steady state data was used. Based on synthetic examples, we expect that multiple transient annealing will be able to discem channel pattems much more effectively than steady state annealing. However, because the estimate is sensitive to flow measurements and it is flow we are predicting, we have confidence that this approach gives answers that make some sense.

As described in 6.2 we used an energy function defined with the relative distance to the observed head values, that is we shifted the boundary conditions to minimize the energy. It is of interest to compare these results to the case where the absolute value of bead difference is used instead. However, at the last iteration the shifi was small (about $1.4 \mathrm{~m})$ so this change in approach will probably not have a large effect on the result. Even more enticing is the idea of using the configuration at each iteration to sequentially model flow into each of the $\mathrm{N}$ - and $\mathrm{W}$-holes. In this case, we could define an energy function which include terms for the difference between predicted and measured flow, as well as head. Thus at each iteration we would nun the flow code five times instead of once. A weighting function would probably be needed in order to account for the fact that the units and magnitude of head differences are different than the units and magnitude of flux differences. In this case, we would expect annealing to be more sensitive to the heterogeneities near the wells which control the inflows, so we would not have had to use the boundary condition shifting. Although this approach is more CPU intensive, it may converge faster and would be worth trying.

Questions remain about weighting some data over others and the effect of different staring configurations of the model. Davey et al (1990) report on such studies and show that the initial percentage of conductors has litue effect on the final percentage. Also, it is not surprising these synthetic cases show much better resolution of pattem when the template reproduces the orientations of the real conductors. This fact suppors the need for the a priori definition of the template using geology and geomechanics approaches.

Finally, these results indicate that we may have over-discretized the channel network of the template. Possibly one of the reasons that the annealing did not result in any pattem definition is that the network simply allowed too many degrees of freedom. There is a need to look at discretization versus information content in the well tests. This also could be done with synthetic examples. 
The inflow to the D-holes has already been measured. After submitting this report, we will compare our predictions to the actual measurements. As the project enters the next stage of work, changes may be made to the hydrologic zones based on new geophysical and geological data. Also, new controlled cross-hole data is now available. This data 4 " t be used to develop a new annealing case. Small scale cross-hole tests have now bec: conducted in the H-zone between the D-holes which will allow us to further define the conductor pattem in the H-zone.

As we will have more than one test to anneal against, the energy for any given configuration will be the sum of the energies calculated for all the different tests. Thus we will sequentially simulate each test in a given configuration to get the total energy. Using the new annealed model we will re-predict the inflow to the D-holes.

\subsection{Excavation Inflow}

The last step will be to predict the inflow to the Validation Drift which is to be excavated through the $D$-holes. To make the prediction of inflow to the drift we will have available the measurement of the inflow into the D-holes. This measurement can be used to calibrate our previous annealed model. From there, we need to modify the models to reflect the changes in conditions caused by excavating the drifi. These excavation effects are the cause of some concem. We can easily account for the change in the inner hydraulic boundary condition: The six wells are simply changed to one large well. However, there are other imporant effects.

Data from the Macropermeability Experiment provides evidence for a low permeability zone near the drift. Figure 8.1 reproduced from Wilson, et al. (1981) shows a semi-log plot of the head profiles measured radially away from the drift. The curves should all pass through a point near the origin because the pressure in the drift is zero. To do this, all the curves would have to take a sharp downward tum as shown by the sketched-in lines. Thus between the first measurement point at about $5 \mathrm{~m}$ and the drift the hydraulic gradient increases much faster than expected for radial flow in a homogeneous medium. This hydraulic behavior is strong evidence for an annulus of decreased permeability within the first $5 \mathrm{~m}$ of the drift. In other words, water is piling up outside of a constriction caused by decreased permeability near the drift.

In the case of the Macropermeability data, the pressure profile slope must change by at least a factor of two (see line \#1 on Figure 8.1), thus the flow is decreased by at least a factor of two. However, data from the Buffer Mass Tes: (BMT) at the same location provided evidence for an annulus of high permeability right next to the drift wall $(\mathbf{R}$. Pusch, personal communication). In this case the pressure profile actually will look like line \#2 on Figure 8.1. The flow rate could then be decreased by almost any amount. As a counter example, inflow to the 3D Drift was approximately the same as inflow in to the 3d Drift pilot hole (Olsson personal communication, 1989). In order to know the effect on flow, we need to know the amount of pressure drop and the distance over which it drops. Currently there are no measurements planned to quantify this effect for the Validation Drift.

Below, we give a discussion of the phenomena that could be effecting the inflow 


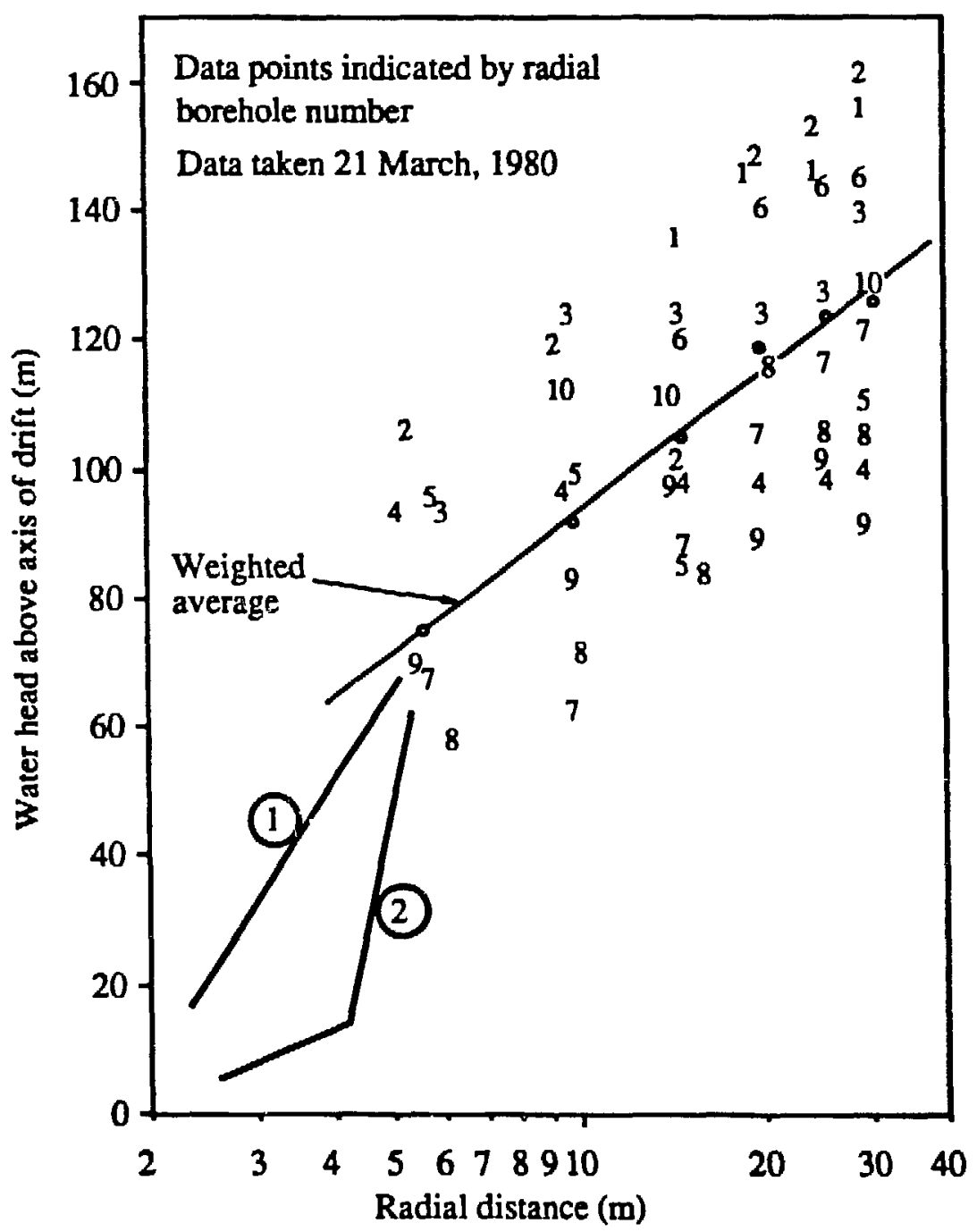

XBL 8011-12708

Figure 8.1. Distance-drawdown plot of the $20^{\circ}$ test results of the macropern:ability experiment. 
followed by a description of data which would provide input to the modeling effon aimed at predicting inflow to the Validation Drifi.

\subsection{Stress Effects}

Stress changes around the drift due to excavation will cause some fractures around the drift to open and some to close. For example, we might expect an increase in hoop stresses affecting an annulus surrounding the drift, this could account for decreased permeability in fractures which are oriented on planes passing through the axis of the drifi. However, fractures sub-parallel to tangents of the drift might tend to open. These sub-parallel fractures might account for the annulus of high permeability around the BMT drift. Net change in inflow could be either up or down depending on how the fractures are interconnected as they enter the drift. So, the stress change may or may not account for head profiles observed in the Macropermeability Experiment.

A calculation is being made of the expected stress changes in three-dimensions based on linear elastic theory (Bengt Stillborg, personal communication). The idea is that these changes can then be resolved with resoect to the orientation of fractures intersecting the drift and then a laboratory based relationship betueen stress and permeability can be used to predict the changes in permeability.

We agree that this stress analysis is useful, but it may not be the best information for predicting the change in permeability. The problem is that the solution depends on the far field state of stress and this is poorly known. Furher, fractures and fracture zones near the drift may cause significant deviations from the linear elastic solution. In application, there will be an error due to the fact that we do not know the actual orientation of the fractures around the drift, so we resolve the wrong stress change and consequent permeability change. Finally, the relationship between stress and permeability will be based on fractures tested in the laboratory and these are small scale samples that are not likely to be the most important fractures. The most imporant fractures from the flow standpoint are in the highly fractured zones and these can not be recovered for laboratory analysis. In sumnary, this approach requires that we make estumates based on estimates that are based on estimates and thus has a high probability of failing.

\subsection{Water Pressure Effects}

A decrease in permeability may occur due to increases in effective stress as the zone around the drift is depressurized. The change in permeability due to further pressure decline during excavation should be minor.

However, if a significant part of the rock around the drift is completely de-pressurized, the "siphon" effect in this region will break down and water flow will only respond to gravity. In other words, the water will not "want" to flow towards the drift, it will only "want" to flow downward (Figure 8.2). In fact, simply creating a zero pressure boundary at the drif wall creates a vertical component in the total head gradient. This means that flow heading towards the drift could be divened vertically past the sides of the drift and this would decrease the flow irito the drift. This effect may have been the reason tracers injected above the 3D-Drift were diverted to other parts of the mine. The effect can easily be handled in the numerical codes if we have pressure measurements near the drift. Currently these are not planned. Planned saline-radar tests may be able to demonstrate that such a diversion of flow has taken place. 


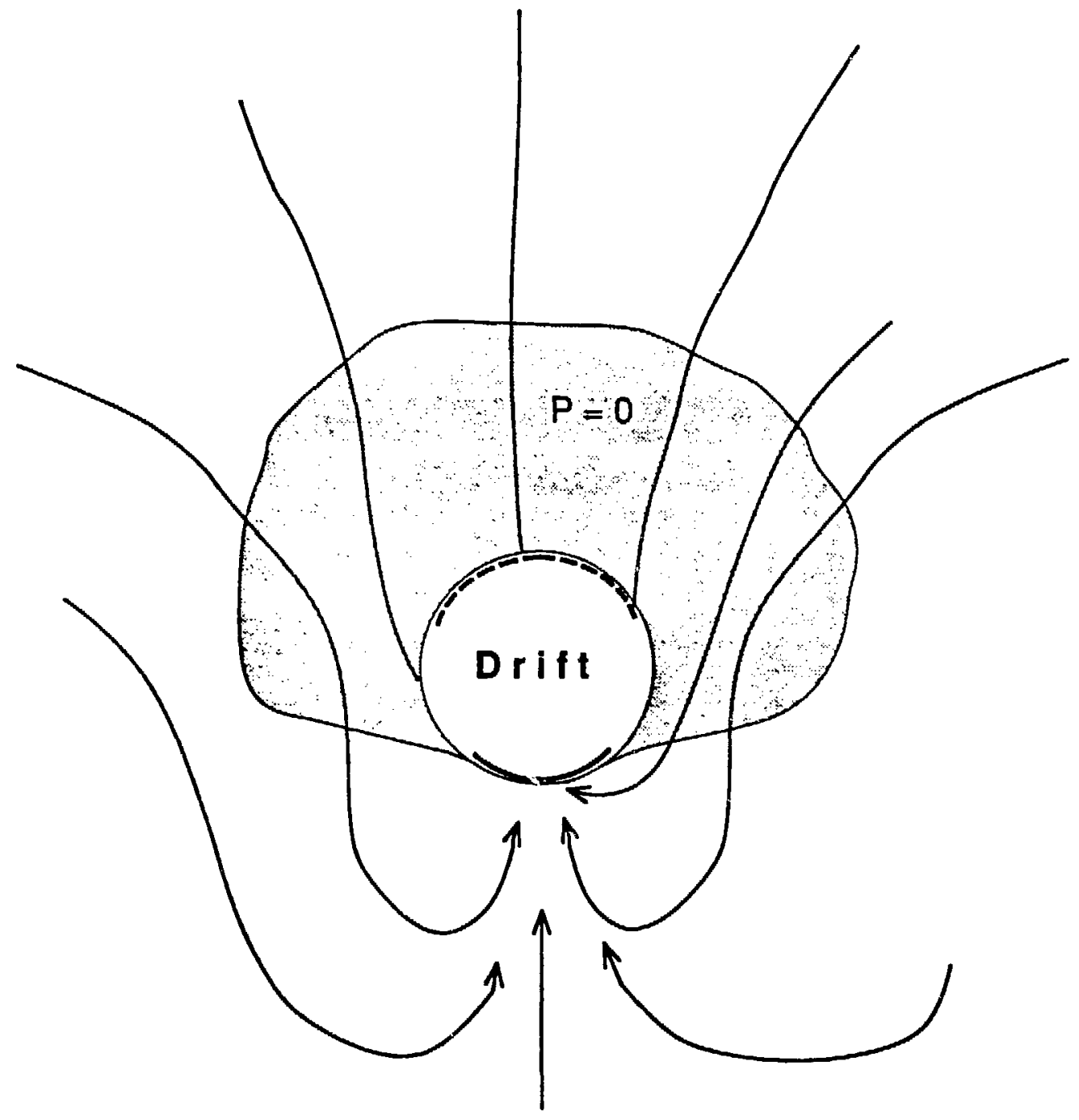

Figure 8.2. Flow diversion due to the de-pressurization. 


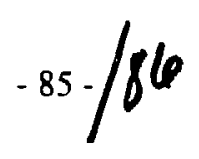

8.4. Blasting Effects

Blasting is expected to produce an annulus of loosened, fractured rock immediately surrounding the drift. Such an annulus would also account for flow parallel to the drift as observed during the Buffer Mass Test. A high permeability zone could slightly enhance flow into the drifi or it could diven flow along the axis and away from the drift.

On the other hand, the explosions can also push air back into the rock The air that gets into the rock will lodge in the larger voids because of capillary effects. As the large voids are the ones most important for flow, a small amount of air can significanuly decrease permeability. An annulus of decreased permeability would decrease the net flow into the drift because it represents a constriction in the flow path. If the low permeability zone is in series with a high permeability zone, the effects of the low permeatility zone will dominate inflow. So, blast injected air could also account for the observations at the Macro-permeability Experiment.

\subsection{Ventilation Effects}

Ventilation can have at least two effects. Ventilation that is too strong can pull water out of the rock, thus increasing the measured inflow. On the other hand, it has recently been shown thai air can enter due to differential drying effects mediated by ventilation (K. Pruess personal communication). This air entry will decrease the permeability around the drift in the same way that air invasion due to explosions will. So ventilation effects could also explain the Macropermeability data discussed above. Further data from the Macro-permeability experiment show that the inflow decreased by about $20 \%$ when the ventilation temperature was increased from 20 to $30^{\circ} \mathrm{C}$. This is evidence for air invasion dominating the effect of ventilation pulling water out of the rock. This may also be the case at the Validation Drift.

\subsection{Conclusion}

Based on the results of the Simulated Drift Experiment we know the inflow' into the Dholes and how it is paritioned. The flow rate into the pan of the D-holes that has subsequently been excavated therefore becomes the best estimate of the flow rate into the drift with out including excavation effects. No experiments are planned to quantify the above excavation effects; we will have to take the effects into account heuristically or by scenario studies. 


\subsection{References}

Barker, J., 1988, "A Generalized Radial-flow Model for Pumping Tests in Fractured Rock," Water Resources Research, 24, (10), 1796-1804.

Banon, C. C., T. A. Schuter, W. R. Page, and J. K. Samuel, 1987. "Computer Generation of Synthetic Fracture Networks for Hydrologic-Flow Modeling," Trans., American Geophysical Union, 68, (44.)

Billaux, D., J. P. Chiles, K. Hestir, J. Long, 1989. "Three-Dimensional Statistical Modeling of a Fractured Rock Mass - An Example for the Fanay-Augeres Mine," Intemational Joumal of Rock Mechanics and Mining Science, special issue on Forced Fluid Flow Through Fractured Rock Masses, in press.

Carlsten, S. 1985. Hydrogeological and Hydrochemical Investigations ir، Boreholes - Compilation of Geologic Data, Stripa Project IR 85-04, SKB, Stockholm, Sweden.

Carlsten, S., O. Olsson, O. Persson, M. Sehlstedt, 1988. Site Characterization and Validation Monitoring of Head in the Stripa Mine During 1987, SKB Report 88-02, Stockholm, Sweden.

Carrera, J. and S. P. Neuman, 1986. "Estimation of Aquifer Parameters Under Transient and Steady State Conditions: 2," Water Resources Research, 22, (2).

Chan, T., V. Guvanasen, and N. Liwlestone, 1981. Numerical Modeling to Assess Possible Influence of the Mine Openings on Far-Field In Situ Stress Measurements at Stripa, Lawrence Berkeley Laboratory, Repon No. LBL-12469, Berkeley, Califomia.

Davey, A.. K. Karasaki, J. C. S. Long, M. Landsfeld, A. Mench and S. Martel, 1990. Analysis of the Hydraulic Data from the MI Experiment, NDC-15, Lawrence Berkeley Laboratory, Repor No. LBL-27864.

Freeze, R. A. and Cherry, J. A., 1979. Groundwater, Prentice-Hall, Inc., Englewood Cliff, N.J.

Kachanov, M., 1987. "Elastic Solids with Many Crack: A Simple Method of Analysis," Int. J. Solids Structures, 23, 23-43.

Hajek, B., 1988. "Cooling Schedules for Optimal Annealing," Mathematics of Operations Research, 13. (2). P311-329.

Hestir, K. and J. C. S. Long, 1989. The Permeability of Homogeneous Two-Dimensional Poisson Fracture Networks, in preparation.

Kachanov, M., 1987. "Elastic Solids with Many Crack: A Simple Method of Analysis," Int. J. Solids Structures, 23, 23-43.

Karasaki, K., 1986. Well Test Analysis in Fractured Media, Ph.D. Thesis, Dept. of Materials Science and Mineral Engineering, University of Califomia at Berkeley.

Karasaki, K., 1988. A New Advection-Dispersion Code for Calculating Transport in Fracture Networks, LBL Repor, in press. 
Kilpatrick, S., D. C. Gelat, M. P. Vecchi, 1983. "Optimization by Simulated Annealing," Science, 220.

Lawn, B. R. and T. R. Wilshaw, 1975. Fracture of Brittle Solids, Cambridge University Press. London.

Long, J. C. S. and P. A. Witherspoon, 1985. "The Relationship of the Degree of Interconnection to Permeability in Fracture Networks," $J$. of Geophys. Res., 90, (B4), 3087-3097.

Mandelbrot, B. B., 1982. The Fractal Geometry of Nature, W. H. Freeman and Company, New York, $468 \mathrm{pp}$.

Marel, S. J., D. D. Pollard, and P. Segall, 1988. "Development of Simple Strike-Slip Fault Zones, Mount Abbot Quadrangle, Sierra Nevada, Califomia," Geol. Soc. Am. Bull., 100, 1451-1465.

Metropolis, N., A. Rosenbluth, M. Rosenbluth, A. Teller, and M. Teller, 1953. "Equations of State Calculations by Fast Computing Machines," J. Chem Phys, 21.

Olsson, O., J. H. Black, C. Cosma, and J. Pihl, 1987, Crosshole Investigations - Final Report, Stripa Project Report 87 -16, SKB, Stockholm, Sweden.

Olsson, O., J. H. Black, J. E. Gale, and D. C. Holmes, 1989. Site Characterization and Validation, Stage 2 - Preliminary Predictions, Swedish Geological Co. Report ID No. TR-89-03.

Olsson, O., J. Eriksson, L. Falk, E. Sandberg, 1988. Site Characterization and Validation Borehole Radar Investigations, Stage I, SKB Repor 88-03, Stockholm, Sweden.

Orbach. R., 1986. “Dynamics of Fractal Networks," Science, 231, 814-819.

Petit, J. P. 1987. "Criteria for the Sense of Movement on Fault Surfaces in Brittle Rocks," $J$. Struct. Geology, 9, 597-608.

Press, W. H. . B. P. Flannery, S. A. Teukolsky, and W. T. Vetterling, 1986. Numerical Recipes. Cambridge University Press.

Rodgers. D. D., 1980. “Analysis of Basin Development Produced by En Echelon Strike Slip Faults," in P. F. Ballance and H.G. Reading (eds.), Sedimentation at Oblique-Slip Margins, Intemational Assoc. of Sedimentologists, Oxford, England.

Segall, P. and D. D. Pollard, 1980. "Mechanics of Discontinuous Faults," J. Geophys. Res., 85. 4337-4350.

Segall, P. and D. D. Pollard, 1983. "Nucleation and Growth of Strike Slip Faults in Granite," $J$. Geophys. Res., 88, 555-568.

Sen, P. N, C. Scala, M. H. Cohen, 1981. "A Self Similar Model for Sedimentary Rocks with Application to the Dielectric Constant of Fused Glass Beads," Geophysics, 46. (5), 781795.

Sibson, R. H., 1981. "Fluid Flow Accompanying Faulting: Field Evidence and Models," in D. W. Simpson and P. G. Richards (eds.), Earthquake Prediction, 593-603, AGU, Washington, D. C.

Sibson, R. H., 1986. “'Brecciation Processes in Fault Zones: Inferences from Earthquake Rupturing," PAGEOPH, 124, 159-175. 
SKB (Swedish Nuclear Fuel an Waste Mangement Co.), 1987. Program for the Stripa Project Phase 3 1986-1991, SKB report 87-09, Stockholm, Sweden

Tarantola, A., 1987. Inverse Problem Theory, Elsevier Science Publishing Company Inc., New York, N.Y.

Tchalenko, J. S., 1970. "Similarities ietween Shear Zones of Different Magnitudes," Geol. Soc. Am. Bull., 81, 1625-1640.

Teufel, L. W., 1987. "Permeability Changes during Shear Deformation of Fractured kock," in I. W. Farmer et al. (eds.), Proceedings of the 28th U. S. Symposium on Rock Mechanics, Rotterdam, Balkema, 473-480.

Wilson, C. R., J. C. S. Long, R. M. Galbraith, K. Karasaki, H. Endo, A. O. Dubois, M. J. McPherson, G. Ramqvist, 1981. Geohydrological Data from the Macropermeability Experiment at Stripa, Sweden, Lawrence Berkeley Laboratory Report LBL-12520, Swedish-American Cooperative Report SAC-37, Berkeley, Ca. 


\section{APPENDIX A: THE GRID MODEL}

One problem that we have with the fracture zone model is that we have evidence from the experiments at the Cross-Hole Site at Stripa that fracture zone is not always a uniform, continuous feature. Also, hydrologic features are not always associated with a geophysical signature (Olsson et al., 1987). These findings are consistent with the observations at the SCV site. As we can not be certain of the correlation between geophysics and hydrology, should evaluate the a priori assumption of fracture zones as part of the model validation. When zones are assumed a priori, annealing is used to define the pattem of conductors within the zones, ie in two dimensions. As an altemative, we propose to build a three-dimensional grid template (Figure A.1). Then we can use annealing with the cross-hole hydrologic tests to define panems of conductors in three-dimensions. In this way we can see if the hydrriogic response itself demands fracture zones as an explanation.

In the zone approach, we only use geophysics to locate zones. But the geophysical data also contains information about variability within zones. Figure A.2 shows how geophysics can produce a tomogram which is in a plane that cuts through a hydrologic zone. Along the trace of the zone in the tomographic plane, the tomogram can show variability that may be related to the variable degree of fracturing. Although the flow is not confined to the plane of the tomogram, the information about variability is still usefull.

In the grid approach, the geophysical information can be used by adding a geophysical term to the energy expression. In other words, we try to coinvert the hydrologic and the geophysical data simultaneously. To do this requires that we be able to forward model the geophysical phenomena and compare the model response to the observed response.

Forward modeling of geophysical phenomena can be very complicated. One might try a simple approach such as assuming that each fracture delays the wave by the same amount. Thus is the wave passes through 100 fractures it will be delayed 100 times more than if it only went through one fracture. Practically, this means we identify the geometry of the rays that were generated in the field. We construct the same rays in the model and count the number of fracture elements that the ray passes through (Figure A.3). We calculate a delay for each fracture such that the average velocity in the model is the same as that observed in the field. Then the $Q$ function for geophysics is the squared difference between the observed travel time and the predicted travel time:

$$
\mathrm{Q}_{\text {geophysics }}=\Sigma\left(\mathrm{T}_{\text {abrerved }}-\mathrm{T}_{\text {predicted }}\right)^{2}
$$

\section{$\mathrm{T}_{\text {predicted }}=$ travel in intact rock $-\mathrm{B}$ (the number of fractures intersected)}

where $B$ is chosen such that the average of the predicted travel times equals the average of the observed travel times:

$$
\left\langle\mathrm{T}_{\text {predicled }}\right\rangle=\left\langle\mathrm{T}_{\text {observed }}\right\rangle
$$

The annealing $Q$ function is the weighted sum of the hydrologic and geophysical $Q$ functions. 


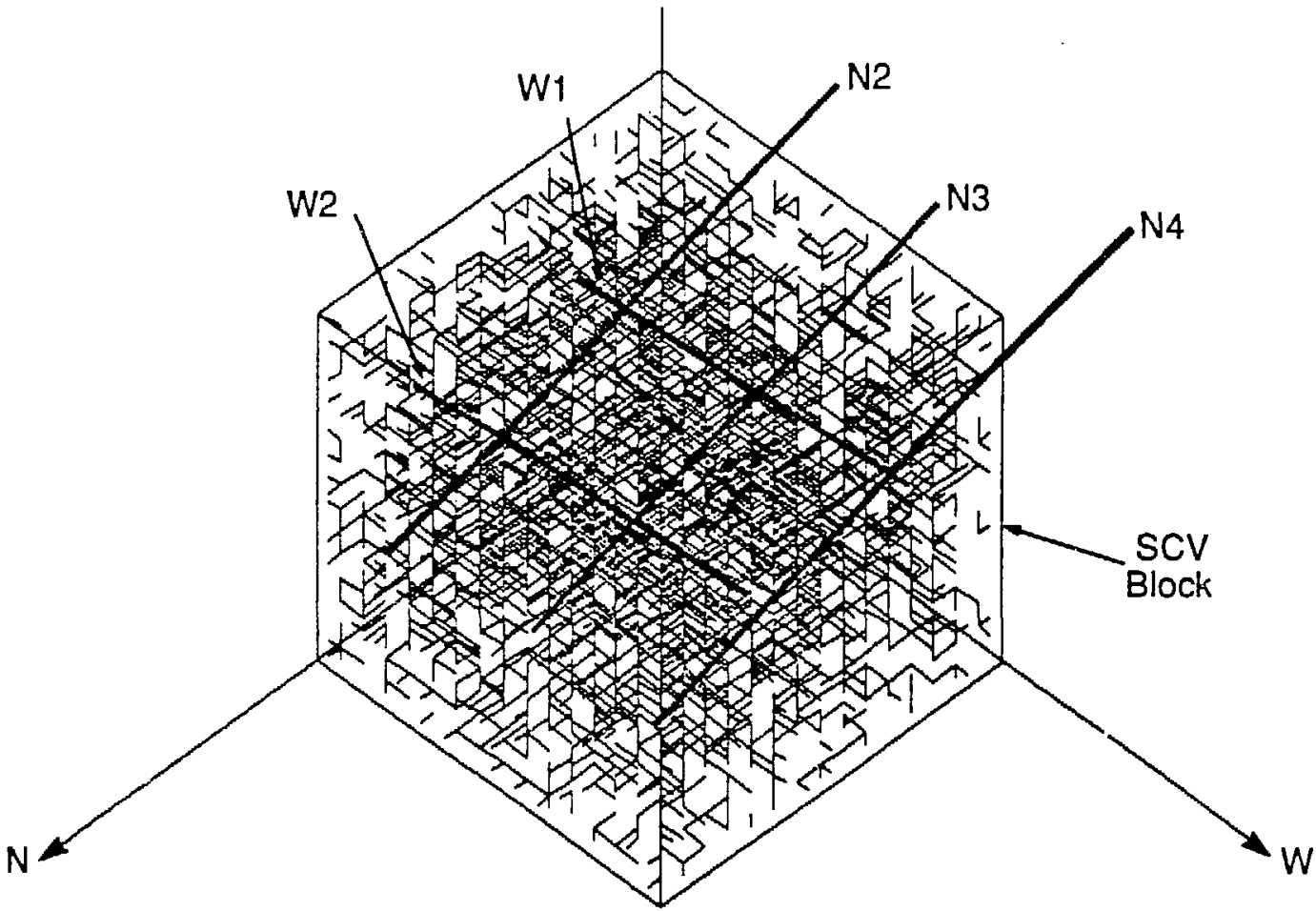

XBL 891.6145

Figure A.1. A partially filled rectangular three-dimensional grid which could be used for co-annealing with geophysics. 


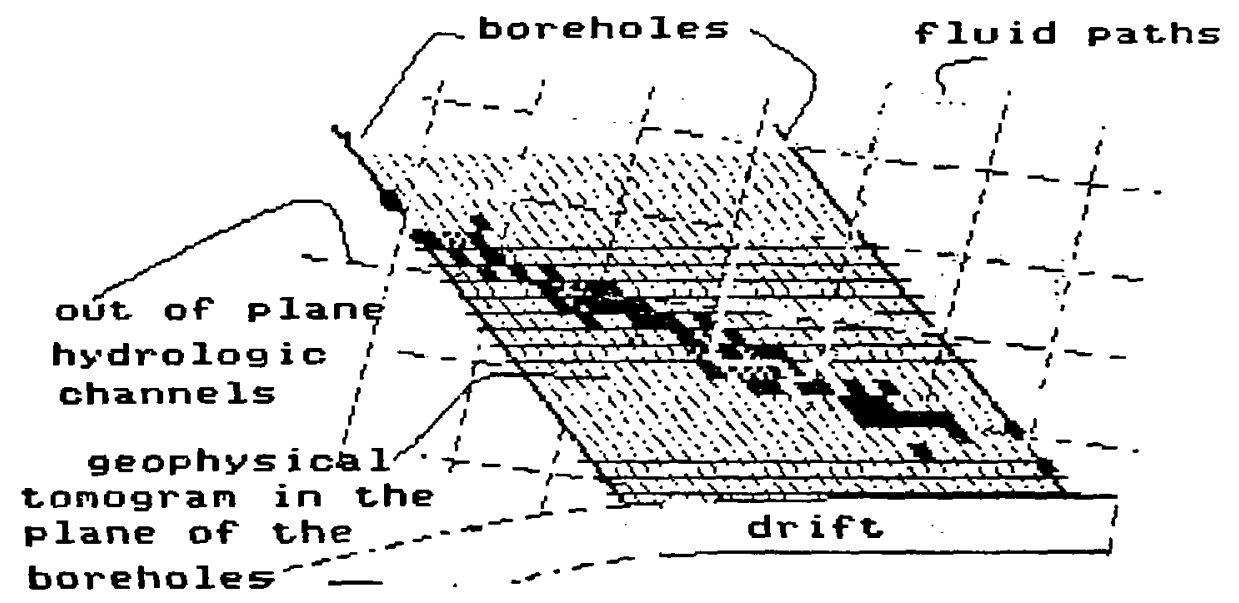

Figure A.2. A cartoon showing a hydrologic zone intersecting the plane of a tomogram. Flow is not confined to the plane of the tomogram, but the tomogram gives information about variability within the hydrologic feature. 

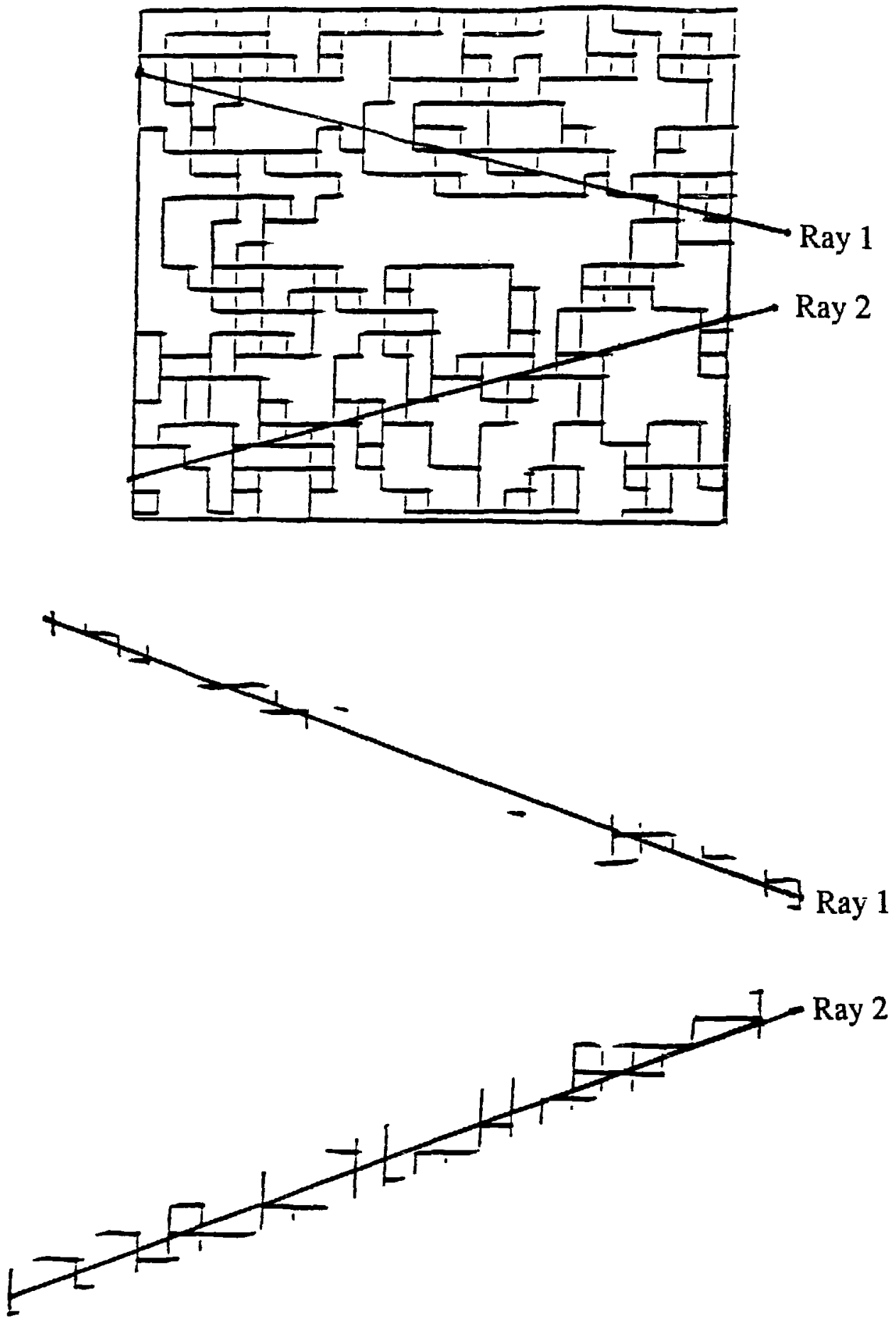

Figure A.3. Ray paths through a network showing how the number of conductors intersected by a ray can be counted. 


$$
\mathrm{Q}=\mathrm{w}_{1} \mathrm{Q}_{\text {hydrologic }}+\mathrm{w}_{2} \mathrm{Q}_{\text {geophysics }}
$$

We take

$$
w_{1} \rightarrow w_{2}
$$

to insure that we match the hydrologic observations preferentially to the geophysical ones.

Now each time a change is examined it will be rejected or accepted based on this total energy. Energy functions could also be obtained for reflection and attenuation data in a similar manner. This approach has the appeal that we make no prior interpretation of the data; we simply let the data speak for itself. 


\section{APPENDIX B: FRACTAL ANALYSIS}

As an altemative to annealing, we can make an attempt to condition the network using a fractal approach to well test interpretation. We can consider that the network of channels in the zone model is a percolating network. If this percolating network is partially filled, it will be characterized by a degree of interconnection. This degree of interconnection is directly related to the permeability of the network (Long and Witherspoon, 1985 and Hestir and Long, 1989). At the same time, such a network is characterized by clusters of conductors which form at scales which exhibit self-similar geometry (Orbach, 1986). Thus, the structure of such networks can be characterized by a fractal dimension (Mandlebrot, 1982). So, if we can determine the fractal dimension of the network, then we know something about how connected the network is. This in tum provides direct information about the percentage of conductors that may be present in the network.

Barker (1988) has provicied a technique for determining the fractal dimension of a network through a well test. He solved the generalized equation of fiow to a well by letting the flow dimension be a variable (Figure B.1). Thereby the flow dimension is allowed to be fractional, say, a dimension of 1.6 or 2.3 (as opposed to integral dimension, i.e.; two or three-dimension space).

The notion of this fractional dimension flow can be easily understood by a simple illustration. Let us consider two points in a space connected by lines representing fractures (Figure B.2). Figure B.2a shows a simple one-dimensional connection between $\mathbf{A}$ and B. Figure B.2b shows a two-dimensional connection between the two points. This is the case when fractures are dense and well connected. However, in reality, fractures are generally irregular and disconnected. Therefore, Figure B.2c is more likely a better representation of the connection between two points. The flow dimension between these two points must lie between one and two. The case can be considered an extension of the discrete composite media cases studied by Karasaki (1986) to a gradually changing media.

The concept of fractional dimension flow can be surnmarized by saying that the area available to flow, $A(r)$ is not necessarily proportional to a integral power of the Euclidian distance $\mathrm{r}$ from the well. In one-dimension, the area available to flow is constant; $A(r) \propto r^{0}$, in two-dimension; $A(r) \propto r^{\jmath}$, and in three-dimension; $A(r) \propto r^{2}$. However, the geometry of the fracture network may be such that $A(r) \propto r^{1.7}$, for example. We believe that the geometry in the fractional dimension flow theory proposed by Barker is a subset of the fractal geometry.

In order to investigate well test behavior in a fractal network, we have generated a simple fracture mesh that has a fractal geometry (Figure B.3). Note the self-similarity of the pattem at different scales. At each scale, five out of nine blocks have fractures of similar pattem. Because each block is divided into $1 / 3$ and five blocks are chosen at each level, the fractal dimension of the mesh is $\ln 5 / \mathrm{ln} 3=1.465$. In this mesh (Figure B.3a) these five blocks are chosen randomlyh. they are chosen randomly. We have also generated symmetric cases. The finite element flow simulator, TRNET was used to simulate well tests in these mesh.

Figure B.2b shows the pressure vs. time curves at the pumped well as well as at several 


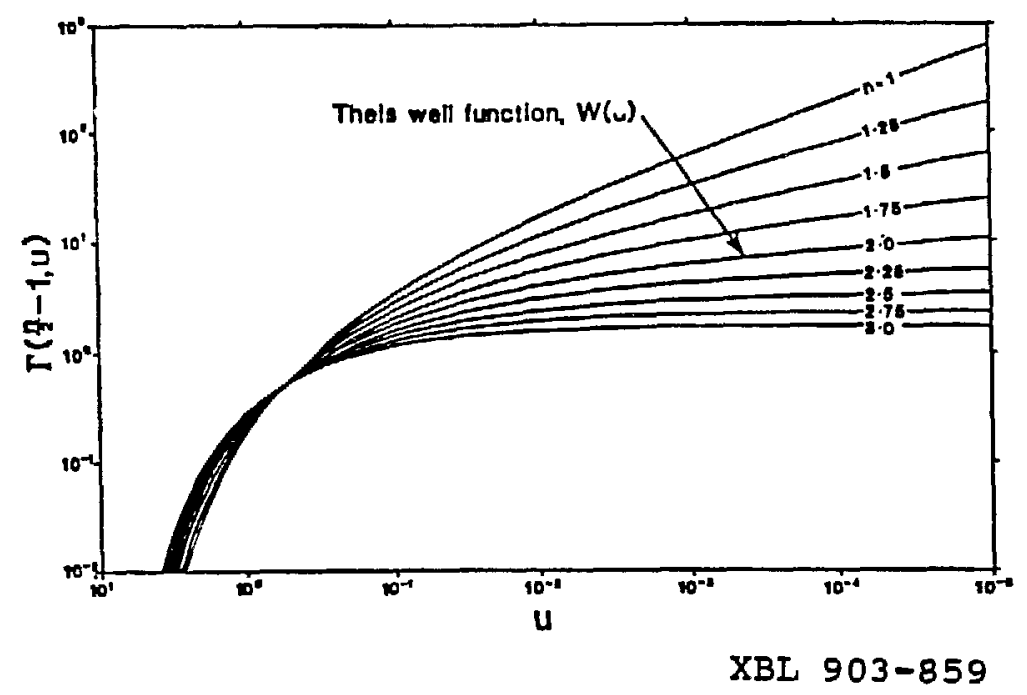

Figure B.1. Drawdown curves for systems of partial dimension (after Barker, 1988).

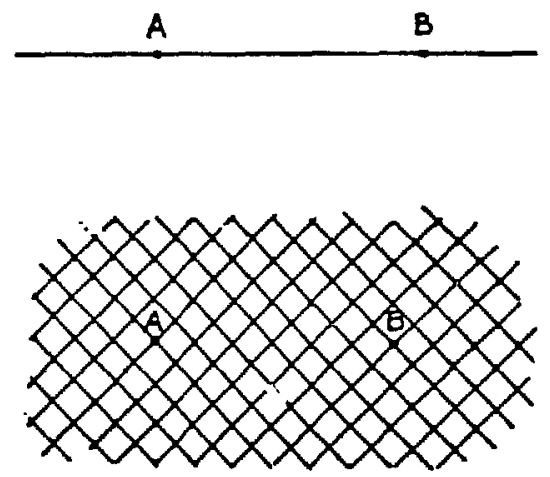

\title{
1-D
}

2-D

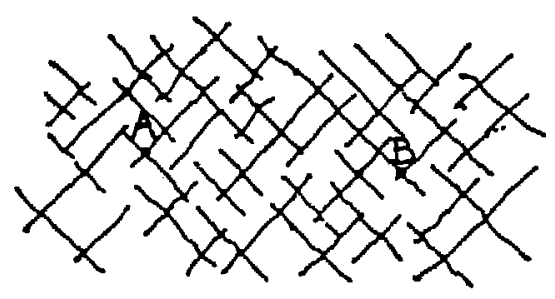

\section{?-D}

\author{
XBL $903-860$
}

Figure B.2. Physical systems where flow between wells $A$ and $B$ would be one-dimensional, two-dimensional, and of partial dimension respectively. 


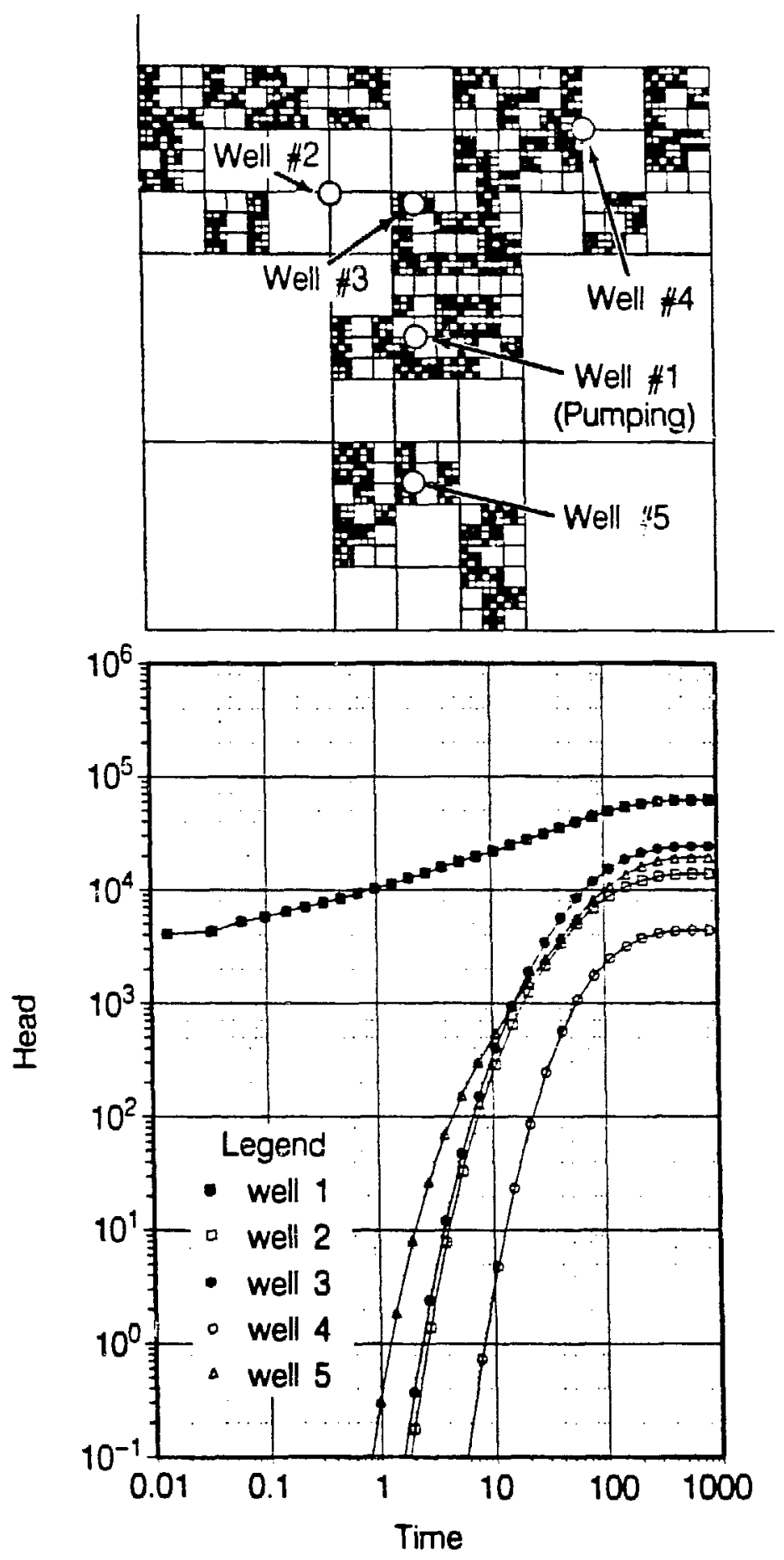

XBL 8916132

Figure B.3. A random fractal mesh and the response curves for a well test on this mesh. 
locations in the corresponding mesh. In a regular orthogonal lattice the curves will be identical to those in an isotropic porous medium. However, as can be seen in the figure, this is not the case. There is a straight line portion in the curves. The slope of the straight line is consistent for both the symmetric and the random case. For the random case, all of the several different realizations produced consistent slopes. Next, by choosing 3, 4, and 6 blocks instead of 5, different meshes with different fractal dimerision were constructed. Again, several realizations were made for each case. It was found that the slope of the straight line portion of the curves is consistent among the realizations and was clearly a function of the fractal dimension (íable B.1). Therefore the slopes are diagnostic of fractal geometry. This is consistent with Barker's theory except for a small difference in the actual number for the slopes predicted by his theory.

Certain fracture systems may have fractal geometry (Barton et al. 1987). Therefore, use of fractal geometry for representing fracture system may be quite plausible. Flow to wells in such geometry differs from that in Euclidian geometry. One should be able to calculate the corresponding fractal dimension from the well test results if the straight line portion is present This may be important information about of the structure of the system. It is also impractical to represent all the fractures explicitly in a numerical model. Fractal representation of fracture geometry may prove promising in that regard also. Fractal analysis can be used to precondition the structure of the channel network at the start of annealing process. This has not been done at this stage of the work, but will be applied to stage 3 data.

Table B.1. Slope of Drawdown Curves for Networks of Different Fractal Dimension

\begin{tabular}{|c|c|c|}
\hline $\begin{array}{c}\text { Number of } \\
\text { Fractured } \\
\text { Squares }\end{array}$ & Fractal Dimension & Average Slope \\
\hline \hline 3 & $\ln 3 / \ln 3=1.000$ & 0.411 \\
4 & $\ln 4 / \ln 3=1.262$ & 0.363 \\
5 & $\ln 5 / \ln 3=1.465$ & 0.323 \\
6 & $\ln 5 / \ln 3=1.631$ & 0.246 \\
\hline
\end{tabular}

